Universidade de Brasília

Instituto de Ciências Exatas

Departamento de Matemática

\title{
Pares de Codazzi em Superfícies de Variedades Homogêneas
}

\author{
por \\ Welinton de Oliveira Gimarez
}

Brasília

2016 


\title{
Pares de Codazzi em superfícies de variedades homogêneas
}

\author{
por \\ Welinton de Oliveira Gimarez *
}

Dissertação apresentada ao Departamento de Matemática da Universidade de Brasilia, como parte dos requisitos para obtenção do grau de

\section{MESTRE EM MATEMÁTICA}

Brasília, 12 de dezembro de 2016.

Comissão Examinadora:
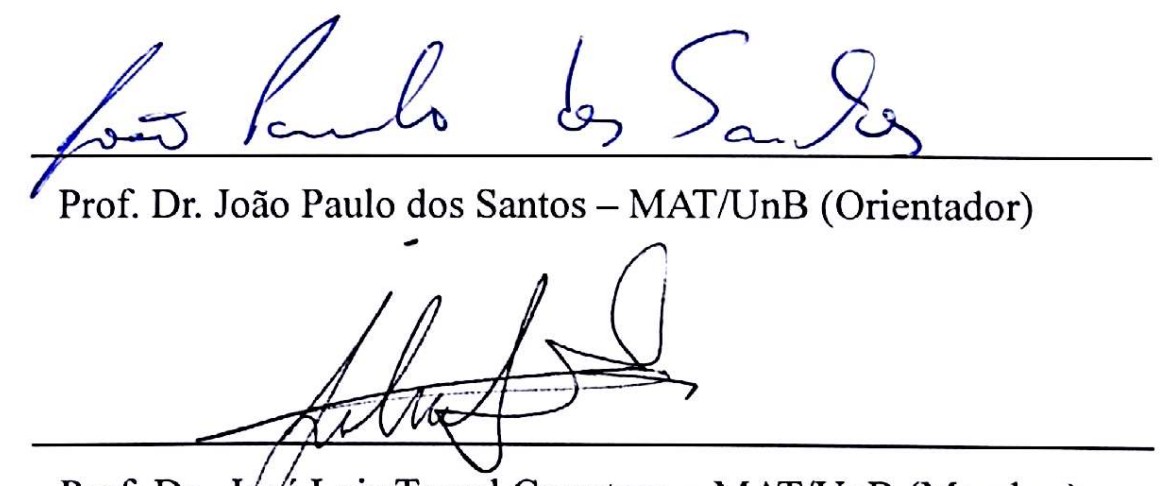

Prof. Dr. Jøsé Luis Teruel Carretero - MAT/UnB (Membro)

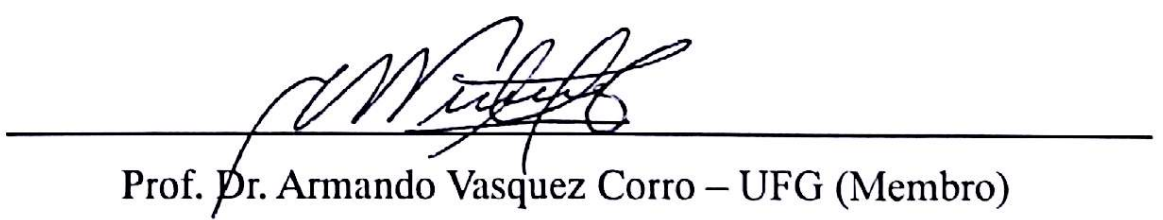

* O autor foi bolsista do $\mathrm{CNPq}$ durante a elaboração desta dissertação. 
Ficha catalográfica elaborada automaticamente, com os dados fornecidos pelo(a) autor(a)

$\mathrm{Gp}$

Gimarez, Welinton de Oliveira

Pares de Codazzi em Superfícies de Variedades Homogêneas / Welinton de Oliveira Gimarez;

orientador João Paulo dos Santos. -- Brasília, 2016. $111 \mathrm{p}$.

Dissertação (Mestrado - Mestrado em Matemática) -Universidade de Brasília, 2016.

1. Pares de Codazzi. 2. Variedades homogêneas. 3. Conjectura de Milnor. 4. Curvatura Gaussiana limitada. 5. Curvatura média constante. I. dos Santos, João Paulo, orient. II. Título. 
"Um dia sem rir é um dia desperdiçado." (Charles Chaplin) 


\section{Agradecimentos}

Meus sinceros agradecimentos a todos que de alguma forma contribuíram para o êxito deste trabalho, e em especial:

- À Deus por me permitir chegar até aqui.

- Aos meus pais, Adhemar e Luiza, pelas orações, apoio e ensinando-me, principalmente, a importância da construção e coerência de meus próprios valores.

- Ao meu irmão, Welton, meu eterno amigo, que soube entender minhas dificuldades e ausências.

- Ao orientador, Professor Dr. João Paulo dos Santos, pela amizade e constante incentivo, sempre indicando a direção a ser tomada. E principalmente pela confiança que depositou em mim. Minha eterna gratidão.

- Ao todos meus professores, pelos valiosos conhecimentos que me forneceram.

- Aos amigos, pelo prazer de suas amizades, conversas, trocas de conhecimentos, ajuda e conselhos.

- Ao $C N P q$, pelo apoio financeiro à este trabalho.

Enfim, agradeço a todos... 


\section{Resumo}

Neste trabalho apresentamos um estudo de pares de Codazzi em superfícies de variedades homogêneas tridimensionais. Inicialmente, apresentamos um resultado abstrato para pares de Codazzi em superfícies completas com curvatura Gaussiana não-positiva e o aplicamos para obter resultados do tipo Efimov e Milnor para superfícies completas nas formas espaciais nãoEuclidianas. Para superfícies de espaços produto, a técnica de pares de Codazzi é utilizada na apresentação de um resultado do tipo Liebmann para superfícies completas com curvatura Gaussiana constante. Nos espaços homogêneos $\mathbb{E}(\kappa, \tau)$, com $\tau \neq 0$, apresentamos um par de Codazzi definido sobre superfícies de curvatura média constante, cuja sua $(2,0)$-parte é a diferencial de Abresch-Rosenberg.

Palavras-Chaves: pares de Codazzi; variedades homogêneas; conjectura de Milnor; curvatura Gaussiana limitada; curvatura Gaussiana constante; curvatura média constante. 


\section{Abstract}

In this work, we present a study of Codazzi pairs on surfaces of 3-dimensional homogeneous manifolds. Initially, we present an abstract result about Codazzi pairs on complete surfaces with non-negative Gauss curvature and we apply it to obtain Efimov and Milnor's type results for complete surfaces in non-Euclidian space forms. For surfaces in product spaces, the technique of Codazzi pairs is applied in the presentation of a Liebmann's type result for complete surfaces with constant Gaussian curvature. In the homogeneous spaces $\mathbb{E}(\kappa, \tau)$, with $\tau \neq 0$, we present a Codazzi pair defined on surfaces with constant mean curvature, whose $(2,0)$-part is the AbreschRosenberg differential.

Keywords: Codazzi pairs; homogeneous manifolds; Milnor's conjecture; Gaussian curvature limited; constant Gaussian curvature; constant mean curvature 


\section{Sumário}

Introdução 1

1 Preliminares 5

1.1 Conjunto de Pares Fundamentais . . . . . . . . . . . . . . . . . 5

1.2 Equações Fundamentais . . . . . . . . . . . . . . . . . . 6

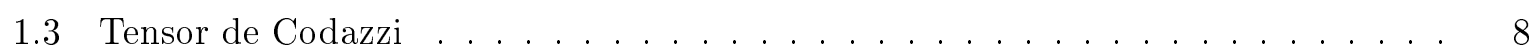

1.4 Imersões Isométricas $\ldots \ldots \ldots \ldots$. . . . . . . . . . . . . . . . . . 11

1.4.1 Segunda Forma Fundamental . . . . . . . . . . . . . . . . . . . . . 11

1.4.2 Equações Fundamentais . . . . . . . . . . . . . . . . . . . . . 12

1.4 .3 Hipersuperfícies . . . . . . . . . . . . . . . . . . . . . 15

1.5 Pares de Codazzi . . . . . . . . . . . . . . . . . . . . . . 19

1.6 Superfície de Riemann e Forma Quadrática Holomorfa . . . . . . . . . . . . . . 22

1.6.1 A diferencial de Hopf . . . . . . . . . . . . . . . . . . . . . . . . . . 32

2 Superfícies Completas com Curvatura Extrínseca Não-positiva em $\mathbb{H}^{3}$ e $\mathbb{S}^{3} \quad 34$

2.1 Introdução . . . . . . . . . . . . . . . . . . . . . . . . . . . . 34

2.2 Definições e Resultados Básicos . . . . . . . . . . . . . . . . . . . 35

2.3 Uma Solução Parcial da Conjectura de Milnor . . . . . . . . . . . . . . . . . 38

2.4 Pares de Codazzi em Superfícies Completas com Curvatura Não-positiva . . . . . 40

2.4.1 Superfícies Completas com $K \leq 0$ em $\mathbb{H}^{3}$ e $\mathbb{S}^{3} \ldots \ldots$. . . . . . . . . . 43

3 Pares de Codazzi nos Espaços Produtos $\quad 47$

3.1 Introdução . . . . . . . . . . . . . . . . . . . . . . . . . 47

3.2 Resultados Básicos . . . . . . . . . . . . . . . . . . . 48

3.2 .1 Equações de compatibilidade . . . . . . . . . . . . . . . . . . 49

3.3 Superfícies de Revolução Completas de Curvatura Constante . . . . . . . . . . . . 53 
3.4 Par de Codazzi para Superfícies de Curvatura Constante em $\mathbb{H}^{2} \times \mathbb{R}$ e $\mathbb{S}^{2} \times \mathbb{R}$. . $\quad 54$

3.5 Teorema Tipo Liebmann . . . . . . . . . . . . . . . . . . . . . . 64

4 Pares de Codazzi no Espaço Homogêneo $\mathbb{E}(\kappa, \tau) \quad 68$

4.1 Introdução . . . . . . . . . . . . . . . . . . . . . . . . . 68

4.2 Resultados Básicos . . . . . . . . . . . . . . . . . . . . . . 69

4.2.1 Variedades Riemannianas Homogêneas $\mathbb{E}(\kappa, \tau) \ldots \ldots \ldots$. . . . . . . 69

4.2 .2 Superfícies Imersas em $\mathbb{E}(\kappa, \tau) \ldots \ldots \ldots \ldots$. . . . . . . . 71

4.2.3 H-Superfícies Imersas em $\mathbb{E}(\kappa, \tau)$ e a diferencial de Abresch-Rosenberg . . 74

4.3 Diferencial Abresch-Rosenberg e Pares de Codazzi . . . . . . . . . . . . . 76

4.3.1 H-Superfícies Imersas em $\mathbb{E}(\kappa, \tau)$ com $\tau=0 \ldots \ldots \ldots \ldots$

4.3.2 H-Superfícies Imersas em $\mathbb{E}(\kappa, \tau)$ com $\tau \neq 0 \ldots \ldots \ldots$. . . . 80

4.4 Classificação para H-Superfícies Imersas em $\mathbb{E}(\kappa, \tau) \ldots \ldots \ldots \ldots$. . . . . 87

5 Conclusão $\quad 92$ 


\section{Introdução}

Uma variedade Riemanniana $M$ é dita homogênea se dados $p, q \in M$ existe uma isometria de $M$ que leva $p$ em $q$. Os espaços homogêneos tridimensionais simplesmente conexos são classificados de acordo com a dimensão do grupo de isometria, que pode ser 3 , 4, ou 6 . No caso da dimensão ser 6 , a variedade é uma forma espacial, ou seja, o espaço Euclidiano $\mathbb{R}^{3}$, a esfera tridimensional $\mathbb{S}^{3}$ ou o espaço hiperbólico tridimensional $\mathbb{H}^{3}$. Se a dimensão do grupo de isometria for 3, a variedade possui a geometria do grupo de $\mathrm{Lie} \mathrm{Sol}_{3}$. Quando a dimensão é 4, a variedade corresponde a uma família 2 -parâmetros, $\kappa, \tau \in \mathbb{R}^{3}, \kappa-4 \tau^{2} \neq 0$, na qual denotamos por $\mathbb{E}(\kappa, \tau)$.

Estas variedades correspondem ao espaço produto $\mathbb{M}^{2}(\kappa) \times \mathbb{R}$ quando $\kappa \neq 0, \tau=0$ onde $\mathbb{M}^{2}$ é uma variedade simplesmente conexa de curvatura constante $\kappa$. Quando $\tau \neq 0$, temos o espaço Heisenberg $\mathrm{Nil}_{3}$ se $\kappa=0$, o espaço de recobrimento universal do grupo linear especial $\widehat{P S L_{2}(\mathbb{R})}$ quando $\kappa<0$ e a esfera de Berger $S_{B}^{3}(\kappa, \tau)$ quando $\kappa>0$.

Um fato importante dos espaços $\mathbb{E}(\kappa, \tau)$ é que eles são uma submersão Riemanniana sobre $\mathbb{M}^{2}$ (superfície de curvatura Gaussiana constante) $\operatorname{com} \tau$ a curvatura do fibrado, sendo as fibras trajetórias de um campo de vetores de Killing unitário definido em $\mathbb{E}(\kappa, \tau)$.

Nesta dissertação, estudamos a equação clássica de Codazzi a partir de um ponto de vista abstrato, e a utilizamos como uma ferramenta analítica para obter resultados globais para superfícies em diferentes ambientes.

A equação Codazzi para uma superfície $\Sigma$ imersa no espaço Euclidiano tridimensional $\mathbb{R}^{3}$ é dada por

$$
\nabla_{X} S Y-\nabla_{Y} S X-S[X, Y]=0, \quad X, Y \in \mathfrak{X}(\Sigma) .
$$

Aqui $\nabla$ é a conexão de Levi- Civita da primeira forma fundamental $I$ e $S$ é o operador forma, definido por $I I(X, Y)=I(S X, Y)$, em que $I I$ é a segunda forma fundamental do superfície. A equação de Codazzi é, juntamente com a equação de Gauss, uma das condições de integrabilidade para superfícies em $\mathbb{R}^{3}$, na qual continuam valendo se substituirmos o espaço ambiente $\mathbb{R}^{3}$ por $\mathbb{S}^{3}$ ou $\mathbb{H}^{3}$. É notável que alguns resultados cruciais da teoria da superfície em $\mathbb{R}^{3}$ só dependem da equação de Codazzi. Este é o caso, por exemplo, do Teorema de Hopf ou Teorema de Liebmann. Isto sugere a possibilidade de adaptar esses resultados a uma definição abstrata de pares de 
Codazzi, isto é, pares de formas quadráticas reais $(I, I I)$ em uma superfície que satisfazem a equação de Codazzi, onde $I$ é uma métrica Riemanniana, e explorar as possíveis consequências superfície.

Inspirados pelos trabalhos de José Gálvez, Antonio Martínez e José Teruel em [21] e [20], estudaremos pares de Codazzi em superfícies e seus invariantes associados, tais como as curvaturas principais, a curvatura média, a curvatura extrínseca e a diferencial de Hopf. Veremos a partir destes trabalhos que a teoria de pares de Codazzi é uma ferramenta vantajosa, uma vez que, a partir de um resultado abstrato para pares de Codazzi em superfícies, serão apresentados resultados tipos Milnor e Efimov para superfícies em $\mathbb{H}^{3}$ e $\mathbb{S}^{3}$.

Apresentaremos também, no decorrer do trabalho, dois lemas de T. Klotz Milnor [25], de suma importância, que relacionam os conceitos de pares de Codazzi com curvatura extrínseca constante positiva ou curvatura média constante e a diferencial de Hopf holomorfa.

Veremos que diferentemente do que ocorre quando $\Sigma$ é imersa isometricamente em uma forma espacial tridimensional, equação de Codazzi não é suficiente para garantir que a usual diferencial de Hopf é holomorfa sobre superfície no espaço homogêneo $\mathbb{E}(\kappa, \tau)$. No entanto, J.A.Aledo, J.M. Espinar e J.A.Gálvez, (ver [5]), obtiveram um novo par Codazzi geométrico $(A, I I)$ sobre superfície em $\mathbb{M}^{2} \times \mathbb{R}$ tal que a $(2,0)$-parte de $I I$ com respeito a estrutura conforme dada pela primeira forma fundamental $A$ é holomorfa.

Para superfícies nos espaços produto, utilizaremos a técnica de pares de Codazzi para estudar superfícies de curvatura Gaussiana constante, seguindo os resultados de Aledo, Espinar e Galvez em [5] e [6]. Caracterizaremos as condições de integrabilidade para superfícies de curvatura Gaussiana constante e, uma vez estabelecidas, definiremos um novo par fundamental na superfície em termos da primeira, segunda formas fundamentais e da função altura. Vamos provar que tal par fundamental é um par de Codazzi de curvatura extrínseca constante quando a superfície tem curvatura Gaussiana constante.

Veremos que esse par de Codazzi é fundamental para obter um Teorema do tipo Liebmann para superfícies completas de curvatura Gaussiana constante $K>0$ em $\mathbb{H}^{2} \times \mathbb{R}$ ou $K>1 \mathrm{em}$ $\mathbb{S}^{2} \times \mathbb{R}$, caracterizando-as, como as esferas rotacionalmente simétricas de curvatura Gaussiana constante.

Para os espaços $\mathbb{E}(\kappa, \tau)$, ressaltamos que apesar da existência de uma diferencial quadrática holomorfa sobre superfície em $\mathbb{E}(\kappa, \tau), \tau \neq 0$, não existia par de Codazzi em superfícies de curvatura média constante imersas nesses espaços. Inspirados pelos estudos de Espinar e Trejos, em [15], veremos que a diferencial Abresch-Rosenberg tem uma interpretação em termos de um par de Codazzi definido em $H$-superfícies neste espaço quando $\tau \neq 0$.

Organizamos o trabalho em cinco capítulos, como segue.

No capítulo 1, fixaremos as notações, definições e resultados fundamentais que serão utilizadas no decorrer do texto. Definimos par fundamental de uma superfície, bem como as curvaturas médias e extrínseca em relação a parametrizações locais e isotérmicas. Veremos o tensor de Codazzi associado a um par fundamental $(I, I I)$. Este por sua vez, juntamente com a teoria de imersões isométricas, será uma ferramenta de grande importância para definirmos pares de 
Codazzi em uma superfície. Veremos a relação entre pontos umbílicos e a diferencial de Hopf. Uma outra ferramenta bastante utilizada, será análise complexa, na qual nos auxiliará na teoria de superfície de Riemann e formas quadráticas holomorfas.

O capitulo 2 é dedicado ao estudo dos trabalhos [21] e [20]. Instigado pelos trabalhos de Hilbert, apresentaremos inicialmente o Teorema de Efimov, no qual nos diz que nenhuma superfície pode ser imersa no espaço Euclidiano tridimensional, tal que na métrica induzida, seja completa e tenha curvatura Gaussiana $K \leq$ const $<0$. Veremos também que apesar dos progressos significativos na compreensão das superfícies com curvatura negativa, questões importantes sugeridas pelo Teorema de Hilbert permanecem sem resposta até hoje. Entre os problemas abertos mencionaremos a Conjectura de Jonh Milnor. Estudaremos a seguinte solução parcial da conjectura de Milnor, obtida por Smyth e Xavier (ver [20], [29]):

$\psi: \Sigma \longrightarrow \mathbb{R}^{3}$ uma superfície imersa isometricamente de curvatura não positiva. Se uma das suas funçôes curvaturas principais $k_{i}^{2}$ satisfaz $k_{i}^{2} \geq$ const $>0$, então $\psi(\Sigma)$ é um cilindro generalizado em $\mathbb{R}^{3}$.

Veremos uma consequência interessante deste resultado para superfícies completas com curvatura de Gauss não-positiva em $\mathbb{R}^{3}$, com curvatura média limitada a partir de zero. O teorema acima propõe uma solução parcial para a conjectura de Milnor, no caso $K \leq 0$. Apresentaremos a demonstração deste teorema usando resultados do espaço Euclidiano, como o teorema Sacksteder. Além disso, apresentaremos suas extensão para as formas espaciais não-Euclidianas, utilizando para isso o teorema de Huber e a teoria de pares de Codazzi. Na verdade, mostraremos que:

Nenhuma superficie pode ser imersa em $\mathbb{H}^{3}$ (resp. $\mathbb{S}^{3}$ ) se é completa na métrica Riemanniana induzida, com curvatura Gaussiana $K \leq-1$ (resp. $K \leq$ const $<0$ ) $e$ uma de suas curvatura principais $k_{i}$ satisfazendo $k_{i}^{2} \geq \varepsilon>0$, para alguma constante $\varepsilon$ positiva.

Sobre a geometria das superfícies com fins de curvatura não-positiva em formas espaciais não-Euclidiana provaremos:

Considere uma imersão completa em $\mathbb{H}^{3}$ (resp. $\mathbb{S}^{3}$ ) que fora de um subconjunto compacto satisfaça:

- a curvatura Gauss $K \leq-1$ (resp. $K \leq$ const $<0$ ) $e$

- $k_{i}^{2} \geq \varepsilon>0, \varepsilon \in \mathbb{R}$ onde $k_{i}$ é uma de suas curvatura principais.

Então, a imersão tem curvatura total finita e, em particular, tem topologia finita e área finita.

No capítulo 3, apresentaremos algumas definições e resultados sobre os espaços $\mathbb{S}^{2} \times \mathbb{R}$ e $\mathbb{H}^{2} \times \mathbb{R}$. Calcularemos as equações de compatibilidade e apresentaremos as superfícies de revolução completas de curvatura constante em $\mathbb{S}^{2} \times \mathbb{R}$ e $\mathbb{H}^{2} \times \mathbb{R}$. Baseados em [5], provaremos que, 
para superfícies de curvatura constante existe um par de Codazzi relacionado à sua métrica induzida, segunda forma fundamental e função altura. A partir deste novo par, apresentaremos um Teorema do tipo Liebmann para superfícies completas de curvatura Gaussiana constante em $\mathbb{H}^{2} \times \mathbb{R}$ e $\mathbb{S}^{2} \times \mathbb{R}$ cuja demonstração é baseada na versão abstrata do Teorema de Liebmann e nos resultados dados por Aledo, Espinar e Gálvez em [6] e Espinar [14]. Além disso, este par tem curvatura extrínseca constante, o que nos dá a existência de uma forma quadrática holomorfa para superfícies de curvatura constante positiva em $\mathbb{H}^{2} \times \mathbb{R}$ e curvatura constante maior que um em $\mathbb{S}^{2} \times \mathbb{R}$. Esse fato, como ocorre para superfícies de curvatura média constante [1], é a chave para obter a caracterização das superfícies completas de curvatura constante. Assim, obteremos um Teorema do tipo de Liebmann, ou seja, provaremos que existe uma única superfície completa de curvatura constante em $\mathbb{H}^{2} \times \mathbb{R}$ e uma única superfície completa de curvatura constante positiva maior que 1 em $\mathbb{S}^{2} \times \mathbb{R}$, a menos de isometrias do espaço ambiente. Estas superfícies completas são precisamente as superfícies de revolução.

O capítulo 4, dedicaremos ao estudo do artigo [15]. Interpretaremos o par de Codazzi da diferencial Abresch-Rosenberg e suas propriedades geométricas. Discutiremos o caso conhecido da $H$-superfície em um espaço produto $\mathbb{M}^{2} \times \mathbb{R}$. Depois, obteremos um par Codazzi geométrico associado a diferencial Abresch-Rosenberg sobre qualquer $H$-superfície imersa em $\mathbb{E}(\kappa, \tau)$ quando $\tau \neq 0$. Finalizaremos o capítulo com algumas definições e resultados de classificação para $H$-superfícies em $\mathbb{E}(\kappa, \tau)$.

No capítulo 5, encontra-se a conclusão do nosso trabalho, na qual apresentaremos um breve resumo dos resultados obtidos. 


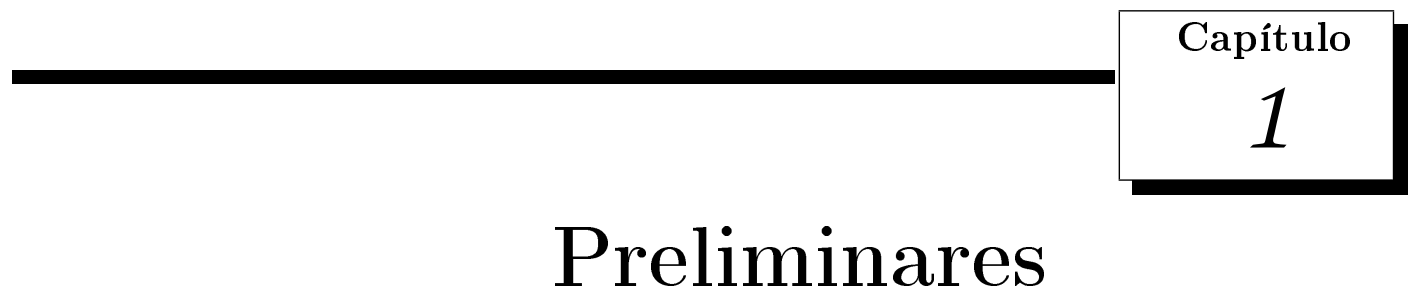

Neste capítulo introduziremos os conceitos básicos utilizados no restante do trabalho. Definiremos um par fundamental de formas quadráticas em uma superfície, inspirados pelas primeira e segunda formas quadráticas de uma superfície em $\mathbb{R}^{3}$. Discutiremos as representações de um par fundamental com respeito a sistemas de coordenadas na superfície, em particular, parâmetros isotérmicos, assim como as representações da complexificação de tais pares em relação aos parâmetros complexos associados. Definiremos as curvaturas média e Gaussiana de um par fundamental e obtemos suas expressões em termos de parâmetros locais. Discutiremos a noção de ponto umbílico de um par fundamental e sua relação com a diferencial de Hopf do par.

Baseado em [4] e [14], começaremos listando vários conceitos básicos e resultados que usaremos no decorrer do trabalho. Iremos supor que a diferenciabilidade é sempre $\mathcal{C}^{\infty}$ e denotaremos por $\Sigma$ uma superfície orientada. Detalhes adicionais podem ser encontrados em [9], [8], [19], [22], [24] e [27].

\subsection{Conjunto de Pares Fundamentais}

Definição 1.1.1. Sejam $\mathcal{Q}(\Sigma)$ o conjunto de formas bilineares simétricas sobre $\Sigma$, que identificaremos como sua forma quadrática associada e $\mathcal{R}(\Sigma)$ o conjunto que define uma métrica Riemanniana. A um par $(I, I I)$ tal que $(I, I I) \in \mathcal{R}(\Sigma) \times \mathcal{Q}(\Sigma) \equiv \mathcal{P}(\Sigma)$ chamaremos par fundamental sobre $\Sigma$. Todo conjunto $\mathcal{P}(\Sigma)$ chamaremos conjunto dos pares fundamentais.

Existe uma correspondência bijetiva entre $\mathcal{Q}(\Sigma)$ e o conjunto $\mathcal{S}(\Sigma,\langle\rangle$,$) de endomorfismo$ autoadjunto de $\mathfrak{X}(\Sigma)$, com respeito à métrica $\langle,\rangle \equiv I$ fixada, isto é,

$$
\mathcal{S}(\Sigma,\langle,\rangle)=\{S: \mathfrak{X}(\Sigma) \longrightarrow \mathfrak{X}(\Sigma) ;\langle S X, Y\rangle=\langle X, S Y\rangle, \forall X, Y \in \mathfrak{X}(\Sigma)\} .
$$

Assim, associada a $S \in \mathcal{S}(\Sigma,\langle\rangle$,$) podemos definir uma forma quadrática, I I_{S}$, por

$$
I I_{S}=\langle S X, Y\rangle, \quad X, Y \in \mathfrak{X}(\Sigma),
$$


e dada $I I \in \mathcal{Q}(\Sigma)$, podemos definir $S \in \mathcal{S}(\Sigma,\langle\rangle$,$) por$

$$
I I(X, Y)=\langle S X, Y\rangle, X, Y \in \mathfrak{X}(\Sigma),
$$

$S$ a qual chamamos operador forma.

Portanto, se fixarmos $\langle,\rangle \in \mathcal{R}(\Sigma)$, podemos considerar endomorfismo autoadjunto ou forma quadrática para definir um par fundamental usando a identificação entre $\mathcal{Q}(\Sigma)$ e $\mathcal{S}(\Sigma,\langle\rangle$,$) . Então,$ um par fundamental sobre $\Sigma$ é equivalente a considerar uma métrica Riemanniana $\langle$,$\rangle em \Sigma$ e um endomorfismo autoadjunto (com respeito a $\langle$,$\rangle ) de \mathfrak{X}(\Sigma)$.

Usaremos a notação distinta em cada caso:

- Para formas quadráticas usaremos $(I, I I)$;

- Para endomorfismo autoadjunto, a notação será $(\langle\rangle, S$,$) .$

Dado um par fundamental $(I, I I) \in \mathcal{P}(\Sigma)$, podemos definir a curvatura média $H$, a curvatura extrínseca $K(I, I I) \equiv K$ e as curvaturas principais $k_{i}, i=1,2$, de $(I, I I)$ como a metade do traço, o determinante e os valores próprios do endomorfismo $S$, respectivamente. Definimos também a curvatura assimétrica de $(I, I I)$ por

$$
q=q(I, I I)=H^{2}-K(I, I I)=\frac{\left(k_{1}-k_{2}\right)^{2}}{4} .
$$

Definição 1.1.2. Diremos que o par fundamental $(I, I I) \equiv(\langle\rangle, S$,$) é umbílico em p \in \Sigma$ (ou $p \in \Sigma$ é ponto umbílico do par $(I, I I)$, se II é proporcional à $I$ em $p$, isto é, $I I=\alpha I$ em $p$, para algum $\alpha$ real.

\subsection{Equações Fundamentais}

É conveniente continuar escrevendo parte do que vimos em um sistema de coordenadas locais. Sejam $(x, y)$ parâmetros locais em torno de $U$ de $\Sigma,(I, I I) \in \mathcal{P}(\Sigma)$ escrevemos

$$
\begin{aligned}
I & =E d x^{2}+2 F d x d y+G d y^{2} \\
I I & =e d x^{2}+2 f d x d y+g d y^{2}
\end{aligned}
$$

onde $E, F, G, e, f, g \in C^{\infty}(U)$. Então, as curvaturas média, extrínseca e principais podem ser definidas em termos dos coeficientes da primeira e segunda formas fundamentais. 
Proposição 1.2.1. Sejam $(U, \varphi=(x, y))$ uma parametrização local em $\Sigma e(I, I I) \in \mathcal{P}(\Sigma)$ tal que

$$
\begin{aligned}
I & =E d x^{2}+2 F d x d y+G d y^{2} \\
I I & =e d x^{2}+2 f d x d y+g d y^{2} .
\end{aligned}
$$

Então, a curvatura média e a curvatura extrínseca são dadas por

$$
\begin{aligned}
H & =H(I, I I)=\frac{E g+G e-2 F f}{2\left(E G-F^{2}\right)} \\
K & =K(I, I I)=\frac{e g-f^{2}}{E G-F^{2}} .
\end{aligned}
$$

Além disso, as curvaturas principais de $(I, I I)$ são

$$
\begin{aligned}
& k_{1}=H+\sqrt{H^{2}-K} \\
& k_{2}=H-\sqrt{H^{2}-K}
\end{aligned}
$$

Demonstração. Ver [8].

Como $(\Sigma, g)$ é uma superfície munida de uma métrica $g$, então existe uma única conexão $\nabla$ afim sobre $\Sigma$, a saber, a conexão de Levi-Civita de $I$.

Denotaremos por $\left\{\partial_{x}, \partial_{y}\right\}$ os campos coordenados associados à parametrização. Então as funções $\Gamma_{i j}^{k}$ definidas em $U$ por

$$
\begin{aligned}
& \nabla_{\partial_{x}} \partial_{x}=\Gamma_{11}^{1} \partial_{x}+\Gamma_{11}^{2} \partial_{y} \\
& \nabla_{\partial_{x}} \partial_{y}=\Gamma_{12}^{1} \partial_{x}+\Gamma_{12}^{2} \partial_{y} \\
& \nabla_{\partial_{y}} \partial_{y}=\Gamma_{22}^{1} \partial_{x}+\Gamma_{22}^{2} \partial_{y}
\end{aligned}
$$

são os coeficientes da conexão $\nabla$ em $U$ associada aos parâmetros $(x, y)$, chamados símbolos de Christoffel associados à métrica $I$ e são dados por

$$
\begin{aligned}
& \Gamma_{11}^{1}=\frac{1}{2\left(E G-F^{2}\right)}\left(G E_{x}-2 F F_{x}+F E_{y}\right) \\
& \Gamma_{11}^{2}=-\frac{1}{2\left(E G-F^{2}\right)}\left(E E_{y}-2 E F_{x}+F E_{x}\right) \\
& \Gamma_{12}^{1}=\frac{1}{2\left(E G-F^{2}\right)}\left(G E_{y}-F G_{x}\right) \\
& \Gamma_{12}^{2}=\frac{1}{2\left(E G-F^{2}\right)}\left(E G_{x}-F E_{y}\right) \\
& \Gamma_{22}^{1}=-\frac{1}{2\left(E G-F^{2}\right)}\left(G G_{x}-2 G F_{y}+F G_{y}\right) \\
& \Gamma_{22}^{2}=\frac{1}{2\left(E G-F^{2}\right)}\left(E G_{y}-2 F F_{y}+F G_{x}\right) .
\end{aligned}
$$


Por outro lado, um elemento de grande importância associado à conexão $\nabla$ é o tensor de curvatura $R$ dado por:

$$
R(X, Y) Z=\nabla_{Y} \nabla_{X} Z-\nabla_{X} \nabla_{Y} Z+\nabla_{[X, Y]} Z, \quad X, Y, Z \in \mathfrak{X}(\Sigma),
$$

que mede o quanto deixa a métrica de ser plana, isto é, tem curvatura zero.

Associada ao tensor de curvatura $R$ está a curvatura seccional de $I$, que é dada por

$$
K(I)(p)=\frac{\left\langle R\left(X_{p}, Y_{p}\right) X_{p}, Y_{p}\right\rangle}{\left\|X_{p} \times Y_{p}\right\|^{2}} \quad \forall p \in S
$$

onde $X_{p}, Y_{p} \in \mathrm{T}_{p} S$ são linearmente independentes e

$$
\left\|X_{p} \times Y_{p}\right\|=\sqrt{\left\|X_{p}\right\|^{2}\left\|Y_{p}\right\|^{2}-\left\langle X_{p}, Y_{p}\right\rangle^{2}} .
$$

Notemos que a definição de $K(I)$ não depende dos vetores escolhidos, e sim dos pontos onde estamos trabalhando. Referimos a $K(I)$ como a curvatura intrínseca ou curvatura de Gauss, quando estivermos trabalhando com superfícies.

Proposição 1.2.2 (Ver [9]). Seja $M$ uma variedade Riemanniana completa com curvatura seccional constante $K(1,0,-1)$. Então $M$ é isométrica a $\widetilde{M} / \Gamma$, onde $\widetilde{M}$ é $S^{n}$ (se $K=1$ ), $R^{n}$ (se $K=0$ ) ou $H^{n}$ (se $K=-1$ ), $\Gamma$ é um subgrupo do grupo das isometrias de $\widetilde{M}$ que opera de modo propriamente descontínuo em $\widetilde{M}$, e a métrica de $\widetilde{M} / \Gamma$ é a induzida pelo recobrimento $\pi: \widetilde{M} \longrightarrow \widetilde{M} / \Gamma$.

\subsection{Tensor de Codazzi}

Definição 1.3.1. Dado $(\langle\rangle, S,) \in \mathcal{P}(\Sigma)$, o tensor de Codazzi associado a $S$ é a aplicação $T_{S}: \mathfrak{X}(\Sigma) \times \mathfrak{X}(\Sigma) \longrightarrow \mathfrak{X}(\Sigma)$ dada por

$$
T_{S}(X, Y)=\nabla_{X} S Y-\nabla_{Y} S X-S[X, Y] ; \quad X, Y \in \mathfrak{X}(\Sigma) .
$$

Vejamos algumas propriedades do Tensor de Codazzi que seguem diretamente da definição.

Proposição 1.3.1. Seja $\langle,\rangle \in \mathcal{R}(\Sigma)$. Então:

1. $T_{S}$ é antissimétrico;

2. $T_{S}$ é multilinear em $\mathcal{C}^{\infty}(\Sigma)$, isto é,

$$
\begin{aligned}
T_{S}\left(f_{1} X_{1}+f_{2} X_{2}\right) & =f_{1} T_{S}\left(X_{1}, Y\right)+f_{2} T_{S}\left(X_{2}, Y\right) \\
T_{S}\left(X, f_{1} Y_{1}+f_{2} Y_{2}\right) & =f_{1} T_{S}\left(X, Y_{1}\right)+f_{2} T_{S}\left(X, Y_{2}\right)
\end{aligned}
$$

$X, X_{1}, X_{2}, Y, Y_{1}, Y_{2} \in \mathfrak{X}(\Sigma)$ e $f_{1}, f_{2} \in \mathcal{C}^{\infty}$. 
3. Dados campos de vetores $X, Y \in \mathfrak{X}(\Sigma)$ e $f \in \mathcal{C}^{\infty}(\Sigma)$, temos

$$
T_{f S}(X, Y)=f T_{S}(X, Y)+X(f) S Y-Y(f) S X .
$$

Demonstração. 1 . Sejam $X, Y \in \mathfrak{X}(\Sigma)$. Como $[X, Y]=-[Y, X]$, então

$$
\begin{aligned}
T_{S}(X, Y) & =\nabla_{X} S Y-\nabla_{Y} S X-S[X, Y] \\
& =-\left(\nabla_{Y} S X-\nabla_{X} S Y+S[X, Y]\right) \\
& =-\left(\nabla_{Y} S X-\nabla_{X} S Y-S[Y, X]\right) \\
& =T_{S}(Y, X) .
\end{aligned}
$$

2. Sejam $X_{1}, X_{2}, Y \in \mathfrak{X}(\Sigma)$ e $f_{1}, f_{2} \in \mathcal{C}^{\infty}$. Temos que

$$
T_{S}\left(f_{1} X_{1}+f_{2} X_{2}, Y\right)=\nabla_{f_{1} X_{1}+f_{2} X_{2}} S Y-\nabla_{Y} S\left(f_{1} X_{1}+f_{2} X_{2}\right)-S\left[f_{1} X_{1}+f_{2} X_{2}, Y\right]
$$

Mas

$$
\begin{gathered}
\nabla_{f_{1} X_{1}+f_{2} X_{2}}=f_{1} \nabla_{X_{1}} S Y+f_{2} \nabla_{X_{2}} S Y \\
\nabla_{Y} S\left(f_{1} X_{1}+f_{2} X_{2}\right)=\nabla_{Y}\left(f_{1} S X_{1}+f_{2} S X_{2}\right) \\
=f_{1} \nabla_{Y} S X_{1}+Y\left(f_{1}\right) S X_{1}+f_{2} \nabla_{Y} S X_{2}+Y\left(f_{2}\right) S X_{2},
\end{gathered}
$$

e

$$
\begin{aligned}
S\left[f_{1} X_{1}+f_{2} X_{2}, Y\right] & =S\left[f_{1} X_{1}, Y\right]+S\left[f_{2} X_{2}, Y\right] \\
& =S\left(f_{1}\left[X_{1}, Y\right]-Y\left(f_{1}\right) X_{1}\right)+S\left(f_{2}\left[X_{2}, Y\right]-Y\left(f_{2}\right) X_{2}\right) \\
& =f_{1} S\left[X_{1}, Y\right]-Y\left(f_{1}\right) S X_{1}+f_{2} S\left[X_{2}, Y\right]-Y\left(f_{2}\right) S X_{2} .
\end{aligned}
$$

Logo,

$$
T_{S}\left(f_{1} X_{1}+f_{2} X_{2}, Y\right)=f_{1} T_{s}\left(X_{1}, Y\right)+f_{2} T_{s}\left(X_{2}, Y\right) .
$$

De forma análoga, obtemos

$$
T_{S}\left(X, f_{1} Y_{1}+f_{2} Y_{2}\right)=f_{1} T_{s}\left(X, Y_{1}\right)+f_{2} T_{s}\left(X, Y_{2}\right) .
$$


3. Sejam $\mathrm{X}, \mathrm{Y} \in \mathfrak{X}(\Sigma)$ e $f \in \mathcal{C}^{\infty}(\Sigma)$, então

$$
\begin{aligned}
T_{f S}(X, Y) & =\nabla_{X} f S Y-\nabla_{Y} f S X-f S[X, Y] \\
& =f \nabla_{X} S Y+X(f) S Y-f \nabla_{Y} S X-Y(f) S X-f S[X, Y] \\
& =f\left(\nabla_{X} S Y-\nabla_{Y} S X-S[X, Y]\right)+X(f) S Y-Y(f) S X \\
& =f T .
\end{aligned}
$$

Será útil escrevermos a equação anterior numa vizinhança coordenada. Dados $(x, y)$ parâmetros locais sobre $U \in \Sigma$ e usando as propriedades vistas do Tensor de Codazzi, é suficiente conhecer como $T_{S}$ atua sobre os campos fundamentais $\left\{\partial_{x}, \partial_{y}\right\}$.

Proposição 1.3.2. Dado $(I, I I) \equiv(\langle\rangle, S,) \in \mathcal{P}(\Sigma)$, seja $(x, y)$ uma parametrização local em $U \subset \Sigma$ tal que I e II são escritas como (1.1). Então

$$
\begin{aligned}
\left\langle T_{S}\left(\partial_{x}, \partial_{y}\right), \partial_{x}\right\rangle & =f_{x}-e_{y}+e \Gamma_{12}^{1}-g \Gamma_{11}^{2}+f\left(\Gamma_{12}^{2}-\Gamma_{11}^{1}\right) \\
\left\langle T_{S}\left(\partial_{x}, \partial_{y}\right), \partial_{y}\right\rangle & =g_{x}-f_{y}+e \Gamma_{22}^{1}-g \Gamma_{12}^{2}+f\left(\Gamma_{22}^{2}-\Gamma_{12}^{1}\right)
\end{aligned}
$$

Demonstração. Se $(\langle\rangle, S,) \in \mathcal{P}(\Sigma)$, então de (1.1)

$$
\begin{aligned}
\left\langle T_{S}\left(\partial_{x}, \partial_{y}\right), \partial_{x}\right\rangle= & \left\langle\nabla_{\partial_{x}} S \partial_{y}-\nabla_{\partial_{y}} S \partial_{x}, \partial_{x}\right\rangle \\
= & \partial_{x}\left\langle S \partial_{y}, \partial_{x}\right\rangle-\left\langle S \partial_{y}, \nabla_{\partial_{x}} \partial_{x}\right\rangle \\
& -\partial_{y}\left\langle S \partial_{x}, \partial_{x}\right\rangle+\left\langle S \partial_{x}, \nabla_{\partial_{y}} \partial_{x}\right\rangle \\
= & f_{x}-\left\langle S \partial_{y}, \Gamma_{11}^{1} \partial_{x}+\Gamma_{11}^{2} \partial_{y}\right\rangle-e_{y} \\
& +\left\langle S \partial_{x}, \Gamma_{12}^{1} \partial_{x}+\Gamma_{12}^{2} \partial_{y}\right\rangle \\
= & f_{x}-e_{y}+e \Gamma_{12}^{1}-g \Gamma_{11}^{2}+f\left(\Gamma_{12}^{2}-\Gamma_{11}^{1}\right) .
\end{aligned}
$$

Analogamente,

$$
\left\langle T_{S}\left(\partial_{x}, \partial_{y}\right), \partial_{y}\right\rangle=g_{x}-f_{y}+e \Gamma_{22}^{1}-g \Gamma_{12}^{2}+f\left(\Gamma_{22}^{2}-\Gamma_{12}^{1}\right) .
$$

A multilinearidade de $T_{S}(X, Y)$ nos permite estender o Tensor de Codazzi à funções diferenciáveis do seguinte modo: tomemos $f \in C^{\infty}(\Sigma)$ e consideremos $S=f I d \in \mathcal{S}(\Sigma,\langle\rangle$,$) . Então,$ como $\nabla$ é livre de torção e $T_{I d}=0$, temos que $T_{f}(X, Y)=X(f) Y-Y(f) X=[X, Y](f)$.

A motivação da definição deste Tensor surge da teoria de superfícies em espaços tridimensionais. Neste caso, se $\Sigma \subset \mathbb{R}^{3}$, sabemos que o operador de Weingarten desta superfície verifica a 
equação de Codazzi, isto é

$$
\nabla_{X} S Y-\nabla_{Y} S X-S[X, Y]=0, \quad X, Y \in \mathfrak{X}(\Sigma),
$$

ou equivalentemente, pela definição anterior,

$$
T_{S}(X, Y)=0, \quad X, Y \in \mathfrak{X}(\Sigma),
$$

o que implica, pela Proposição 1.3.2, as equações usuais de Codazzi-Mainardi.

\subsection{Imersões Isométricas}

Esta seção foi baseada no Capítulo VI de [9].

Definição 1.4.1. Dada uma imersão isométrica $f: M^{n} \longrightarrow \bar{M}^{n+m}$, para cada $p \in M$, o produto interno em $T_{p} \bar{M}$ decompõe este espaço na soma direta $T_{p} \bar{M}=T_{p} M \oplus\left(T_{p} M\right)^{\perp}$, onde $\left(T_{p} M\right)^{\perp}$, é o complemento ortogonal de $T_{p} M$ em $T_{p} \bar{M}$. Logo, se $v \in T_{p} \bar{M}$, podemos escrever $v=v^{T}+v^{\perp}$, em que $v^{T} \in T_{p} M$ é chamada componente tangencial de $v$ e $v^{\perp} \in T_{p} M^{\perp}$ é chamada a componente normal de $v$.

Proposição 1.4.1. Seja $\bar{\nabla}$ a conexão Riemanniana de $\bar{M}$. Se $X, Y \in \mathfrak{X}(M)$, então $\nabla_{X} Y=$ $\left(\bar{\nabla}_{\bar{X}} \bar{Y}\right)^{\perp}$, onde $\bar{X}, \bar{Y}$ são quaisquer extensões locais de $X, Y$ a $\bar{M}$, define a conexão Riemanniana associada à métrica induzida de $M$.

\subsubsection{Segunda Forma Fundamental}

Nesta seção compararemos a conexão Riemanniana de $M$ com a conexão Riemanniana de $\bar{M}$ através da segunda forma fundamental. Se $X, Y \in \mathfrak{X}$, então

$$
\bar{\nabla}_{\bar{X}} \bar{Y}=\left(\bar{\nabla}_{\bar{X}} \bar{Y}\right)^{T}+\left(\bar{\nabla}_{\bar{X}} \bar{Y}\right)^{\perp}=\nabla_{X} Y+\left(\bar{\nabla}_{\bar{X}} \bar{Y}\right)^{\perp}
$$

de modo que

$$
\left(\bar{\nabla}_{\bar{X}} \bar{Y}\right)^{\perp}=\bar{\nabla}_{\bar{X}} \bar{Y}-\nabla_{X} Y
$$

é um campo local em $\bar{M}$ normal à $M$.

Definição 1.4.2. A segunda forma fundamental de $M$ é a aplicação $B: \mathfrak{X}(M) \times \mathfrak{X}(M) \longrightarrow$ $N(M)$ definida por $B(X, Y)=\left(\bar{\nabla}_{\bar{X}} \bar{Y}\right)^{\perp}$, onde $\bar{X}, \bar{Y}$ são extensões locais de $X, Y$ a $\bar{M}$.

Proposição 1.4.2. A Segunda Forma Fundamental está bem definida, é bilinear e simétrica.

Como $B$ é bilinear, concluímos, exprimindo $B$ em um sistema de coordenadas, que o valor $B(X, Y)(p)$ depende apenas de $X(p)$ e $Y(p)$. 
Agora podemos definir a Segunda Forma Fundamental segundo o vetor normal $\eta \in\left(T_{p} M\right)^{\perp}$. Seja $p \in M$. Definimos a aplicação $H_{\eta}: T_{p} M \times T_{p} M \longrightarrow \mathbb{R}$ dada por

$$
H_{\eta}(x, y)=\langle B(x, y), \eta\rangle
$$

$x, y \in T_{p} M$.

Definição 1.4.3. A forma quadrática $I I_{\eta}$ definida em $T_{p} M$ por

$$
I I_{\eta}(x)=H_{\eta}(x, x)
$$

é chamada Segunda Forma Fundamental de $f$ em $p$, segundo o vetor normal $\eta$.

Note que pela proposição anterior, $I I_{\eta}$ é bilinear e simétrica. A aplicação bilinear $I I_{\eta}$ está associada à uma aplicação linear autoadjunta $S_{\eta}: T_{p} M \longrightarrow T_{p} M$ dada por

$$
\left\langle S_{\eta}(x), y\right\rangle H_{\eta}(x, y)=\langle B(x, y), \eta\rangle .
$$

Proposição 1.4.3. Sejam $p \in M, x \in T_{p} M$ e $\eta \in\left(T_{p}\right)^{\perp}$. Seja $N$ uma extensão local de $\eta$ normal a $M$. Então $S_{\eta}(x)=-\left(\bar{\nabla}_{x} N\right)^{T}$.

\subsubsection{Equações Fundamentais}

Dada uma imersão isométrica $f: M^{n} \longrightarrow \bar{M}^{n+m}$, temos, em cada $p \in M$, a decomposição $T_{p} \bar{M}=T_{p} M \oplus\left(T_{p} M\right)^{\perp}$, que varia diferenciavelmente com $p$. Isto significa que, localmente, a parte do fibrado tangente $T \bar{M}$, que se projeta sobre $M$, se decompõe em um fibrado tangente $T M$ e em um fibrado normal $T M^{\perp}$.

Dados $X$ e $\eta$, sabemos que $\left(\bar{\nabla}_{X} \eta\right)^{T}=-S_{\eta}(X)$. Agora estudaremos o componente normal de $\bar{\nabla}_{X} \eta$, que será chamada a conexão normal $\nabla^{\perp}$ da imersão. Notemos que

$$
\nabla_{X}^{\perp} \eta=\left(\bar{\nabla}_{X} \eta\right)^{N}=\bar{\nabla}_{X} \eta-\left(\bar{\nabla}_{X} \eta\right)^{T}=\bar{\nabla}_{X} \eta+S_{\eta}(X)
$$

Verificamos facilmente que a conexão normal $\nabla^{\perp}$ possui as propriedades usuais de uma conexão, isto é, é linear em $X$, aditiva em $\eta$, e

$$
\nabla_{X}^{\perp}(f \eta)=f \nabla_{X}^{\perp} \eta+X(f) \eta
$$

$f \in D(M)$. 
De maneira análoga ao caso do fibrado tangente, introduzimos a partir de $\nabla^{\perp}$ uma noção de curvatura no fibrado normal que é chamada curvatura normal $R^{\perp}$ da imersão e definida por

$$
R^{\perp}(X, Y) \eta=\nabla_{Y}^{\perp} \nabla_{X}^{\perp} \eta-\nabla_{X}^{\perp} \nabla_{Y}^{\perp} \eta+\nabla_{[X, Y]}^{\perp} \eta
$$

Proposição 1.4.4 (Equação de Weingarten). Sejam $X, Y \in \mathfrak{X}(M)$ e $N \in\left(T_{p} M\right)^{\perp}$. Então, em $M$ vale $\left\langle\bar{\nabla}_{\bar{X}} \bar{N}, \bar{Y}\right\rangle=-\langle N, B(X, Y)\rangle$, onde $\bar{X}, \bar{Y}, \bar{N}$ são quaisquer extensões locais de $X, Y, N$ a $\bar{M}$.

Demonstração. Como $\langle\bar{N}, \bar{Y}\rangle=0$ em $M$ e $X$ é tangente a $M$, temos

$$
\bar{X}\langle\bar{N}, \bar{Y}\rangle=0
$$

Mas em $M$,

$$
\begin{aligned}
\bar{X}\langle\bar{N}, \bar{Y}\rangle & =\left\langle\bar{\nabla}_{\bar{X}} \bar{N}, \bar{Y}\right\rangle+\left\langle\bar{N}, \overline{\left.\nabla_{\bar{X}} \bar{Y}\right\rangle}\right. \\
& =\left\langle\bar{\nabla}_{\bar{X}} \bar{N}, \bar{Y}\right\rangle+\left\langle\bar{N}, B(X, Y)+\nabla_{X} Y\right\rangle \\
& =\left\langle\bar{\nabla}_{\bar{X}} \bar{N}, \bar{Y}\right\rangle+\langle\bar{N}, B(X, Y)\rangle+\left\langle\bar{N}, \nabla_{X} Y\right\rangle \\
& =\left\langle\bar{\nabla}_{\bar{X}} \bar{N}, \bar{Y}\right\rangle+\langle\bar{N}, B(X, Y)\rangle .
\end{aligned}
$$

Proposição 1.4.5 (Equação de Gauss). Para todo $X, Y, Z, W \in T_{p} M$ vale

$$
\bar{R}(X, Y, Z, T)=R(X, Y, Z, T)+\langle B(X, T), B(Y, Z)\rangle-\langle B(Y, T), B(X, Z)\rangle .
$$

Demonstração. Observe que

$$
\bar{\nabla}_{X} Y=\nabla_{X} Y+B(X, Y)
$$

e

$$
\bar{\nabla}_{X} \eta=\nabla_{X}^{\perp} \eta-S_{\eta}(X)
$$

Então

$$
\begin{aligned}
\bar{R}(X, Y) Z= & \bar{\nabla}_{Y} \bar{\nabla}_{X} Z-\bar{\nabla}_{X} \bar{\nabla}_{Y} Z+\bar{\nabla}_{[X, Y]} Z \\
= & \bar{\nabla}_{Y}\left(\nabla_{X} Z+B(X, Z)\right)-\bar{\nabla}_{X}\left(\nabla_{Y} Z+B(Y, Z)\right) \\
& +\nabla_{[X, Y]} Z+B([X, Y], Z) \\
= & \nabla_{Y} \nabla_{X} Z+B\left(Y, \nabla_{X} Z\right)+\nabla_{Y}^{\perp} B(X, Y)-S_{B(X, Y)} Y \\
& -\nabla_{X} \nabla_{Y} Z+B\left(X, \nabla_{Y} Z\right)-\nabla_{X}^{\perp} B(Y, Z)+S_{B(Y, Z)} X \\
& +\nabla_{[X, Z]} Z+B([X, Y], Z) \\
= & R(X, Y) Z+B\left(Y, \nabla_{X} Z\right)+\nabla_{Y}^{\perp} B(X, Y)-S_{B(X, Z)} Y \\
& -B\left(X, \nabla_{Y} Z\right)-\nabla_{X}^{\perp} B(Y, Z)+S_{B(Y, Z)} X+B([X, Y], Z) .
\end{aligned}
$$


Fazendo o produto interno da última expressão com $T$, obtemos:

$$
\langle\bar{R}(X, Y) Z, T\rangle=\langle R(X, Y) Z, T\rangle-\left\langle S_{B(X, Z)} Y, T\right\rangle+\left\langle S_{B(Y, Z)} X, T\right\rangle .
$$

Como $\left\langle S_{\eta}(x), y\right\rangle=\langle B(x, y), \eta\rangle$, temos

$$
\langle\bar{R}(X, Y) Z, T\rangle=\langle R(X, Y) Z, T\rangle-\langle B(Y, T), B(X, Z)\rangle+\langle B(X, T), B(Y, Z)\rangle .
$$

Corolário 1.4.1. Se $p \in M$ e $X, Y \in T_{p} M$ são vetores ortonormais, vale

$$
K(X, Y)-\bar{K}(X, Y)=\langle B(X, Y), B(X, Y)\rangle-|B(X, Y)|^{2}
$$

Proposição 1.4.6 (Equação de Ricci).

$$
\langle\bar{R}(X, Y) \eta, \xi\rangle=\left\langle R^{\perp}(X, Y) \eta, \xi\right\rangle+\left\langle S_{\eta}\left(S_{\xi} X\right), Y\right\rangle-\left\langle S_{\xi}\left(S_{\eta} X\right), Y\right\rangle
$$

Demonstração.

$$
\begin{aligned}
& \bar{R}(X, Y) \eta=\bar{\nabla}_{Y} \bar{\nabla}_{X} \eta-\bar{\nabla}_{X} \bar{\nabla}_{Y} \eta+\bar{\nabla}_{[X, Y]} \eta \\
& =\bar{\nabla}_{Y}\left(\nabla_{X}^{\perp} \eta-S_{\eta}(X)\right)-\bar{\nabla}_{X}\left(\nabla_{Y}^{\perp} \eta-S_{\eta}(Y)\right)+\nabla_{[X, Y]}^{\perp} \eta-S_{\eta}([X, Y]) \\
& =\nabla_{Y}^{\perp} \nabla_{X}^{\perp} \eta-S_{\nabla_{X} \eta} Y-\nabla_{Y} S_{\eta}(X)-B\left(Y, S_{\eta}(X)\right)-\nabla_{X}^{\perp} \nabla_{Y}^{\perp} \eta+S_{\nabla_{Y}^{\perp} \eta} X \\
& +\nabla_{X} S_{\eta}(Y)+B\left(X, S_{\eta}(Y)\right)+\nabla_{[X, Y]}^{\perp} \eta-S_{\eta}([X, Y]) \\
& =R^{\perp}(X, Y) \eta-B\left(X, S_{\eta}(Y)\right)-B\left(Y, S_{\eta}(X)\right)-S_{\nabla_{X}^{\perp} \eta} Y-\nabla_{Y} S_{\eta}(X) \\
& +S_{\nabla_{Y}^{\perp} \eta} X+\nabla_{X} S_{\eta}(Y)-S_{\eta}[X, Y] .
\end{aligned}
$$

Fazendo o produto interno $\operatorname{com} \xi$

$$
\begin{aligned}
\langle\bar{R}(X, Y) \eta, \xi\rangle & =\left\langle R^{\perp}(X, Y) \eta, \xi\right\rangle+\left\langle B\left(X, S_{\eta}(Y), \xi\right\rangle-\left\langle B\left(Y, S_{\eta}(X), \xi\right\rangle\right.\right. \\
& =\left\langle R^{\perp}(X, Y) \eta, \xi\right\rangle+\left\langle S_{\eta}(X), S_{\eta}(Y)\right\rangle-\left\langle S_{\eta}(Y), S_{\eta}(X)\right\rangle .
\end{aligned}
$$

Agora vamos obter a equação de Codazzi. Sejam $\mathfrak{X}(M)^{\perp}$ o conjunto de campos normais e $B: \mathfrak{X}(M) \times \mathfrak{X}(M) \times \mathfrak{X}(M)^{\perp} \longrightarrow D(M)$ tal que

$$
B(X, Y, \eta)=\langle B(X, Y), \eta\rangle
$$

Definamos

$$
\left(\bar{\nabla}_{X} B\right)(Y, Z)=\bar{\nabla}_{X}(B(Y, Z))-B\left(\nabla_{X} Y, Z\right)-B\left(Y, \nabla_{X} Z\right),
$$

onde $\bar{\nabla} B(Y, Z) \in \mathfrak{X}(M)^{\perp}$ e $X \in T M$. 
Proposição 1.4.7 (Equação de Codazzi). Com a notação acima

$$
\langle\bar{R}(X, Y) Z, \eta\rangle=\left(\bar{\nabla}_{Y} B\right)(X, Z, \eta)-\left(\bar{\nabla}_{X} B\right)(Y, Z, \eta)
$$

Demonstração.

$$
\begin{aligned}
& \langle\bar{R}(X, Y) Z, \eta\rangle=\left\langle\bar{\nabla}_{Y} \bar{\nabla}_{X} Z-\bar{\nabla}_{X} \bar{\nabla}_{Y} Z+\bar{\nabla}_{[X, Y]} Z, \eta\right\rangle \\
& =\left\langle\bar{\nabla}_{Y}\left(\nabla_{X} Z+B(X, Z)\right)-\bar{\nabla}_{X}\left(\nabla_{Y} Z+B(Y, Z)\right)\right. \\
& \left.+\nabla_{[X, Y]} Z+B([X, Y], Z), \eta\right\rangle \\
& =\left\langle B\left(Y, \nabla_{X} Z\right)-B\left(X, \nabla_{Y} Z\right)+\bar{\nabla}_{Y} B(X, Y)-\bar{\nabla}_{X} B(Y, Z)\right. \\
& \left.+B\left(\nabla_{X} Y-\nabla_{Y} X, Z\right), \eta\right\rangle \\
& =\left\langle\bar{\nabla}_{Y} B(X, Z)-B\left(\nabla_{Y} X, Z\right)-B\left(X, \nabla_{Y} Z\right)-\right. \\
& \left.\left(\bar{\nabla}_{X} B(Y, Z)-B\left(\nabla_{X} Y, Z\right)-B\left(Y, \nabla_{X} Z\right)\right), \eta\right\rangle \\
& =\left\langle\left(\bar{\nabla}_{Y} B\right)(X, Y), \eta\right\rangle-\left\langle\left(\bar{\nabla}_{X} B\right)(Y, Z), \eta\right\rangle \\
& =\left(\bar{\nabla}_{Y} B\right)(X, Z, \eta)-\left(\bar{\nabla}_{X} B\right)(Y, Z, \eta) .
\end{aligned}
$$

Observação 1.4.1. Se o espaço ambiente $\bar{M}$ tem curvatura constante, a equação de Codazzi se reduz a:

$$
\left(\bar{\nabla}_{Y} B\right)(X, Z, \eta)=\left(\bar{\nabla}_{X} B\right)(Y, Z, \eta) .
$$

\subsubsection{Hipersuperfícies}

Definição 1.4.4. Se a codimensão da imersão isométrica $i: M^{n} \longrightarrow \bar{M}^{n+k}$ é 1, isto é, $K=1$, dizemos que $M$ é uma Hipersuperfície.

Tomando dois vetores $x, y$ ortonormais vimos, em decorrência da equação de Gauss, que

$$
K(x, y)-\bar{K}(x, y)=\langle B(x, x), B(y, y)\rangle-|B(x, y)|^{2} .
$$

Para o caso de hipersuperfície, a equação acima admite uma forma mais simples.

Quando $M$ é uma hipersuperfície, existem apenas duas escolhas para o vetor unitário normal. Se $M$ e $\bar{M}$ são ambas orientáveis e escolhemos orientação para $M$ e $\bar{M}$, então temos uma escolha única para o vetor unitário normal: se $\left\{e_{1}, \ldots, e_{n}\right\}$ é uma base ortonormal orientada de $T_{p} M$, escolhemos $\eta$ de tal forma que $\left\{e_{1}, \ldots, e_{n}, \eta\right\}$ é uma base ortonormal orientada de $T_{p} \bar{M}$. Isso produz um campo vetorial normal diferenciável em $M$. Esta escolha fixa a segunda forma fundamental e podemos nos referir, simplesmente, a segunda forma fundamental $H_{\eta}$ de $M$ e ao operador forma $S_{\eta}$ de $M$. Como $S$ é autoadjunto, existe uma base ortonormal orientada $\left\{e_{1}, \ldots, e_{n}\right\}$ de $T_{p} M$ 
formada por autovetores, isto é, $S_{\eta}\left(e_{i}\right)=\lambda_{i} e_{i}$, onde $e_{i}$ são as direções principais e os $\lambda_{i}=k_{i}$ curvaturas principais.

Proposição 1.4.8. Se $M$ é uma hipersuperfície,

$$
K\left(e_{i}, e_{j}\right)-\bar{K}\left(e_{i}, e_{j}\right)=\lambda_{i} \lambda_{j}
$$

Demonstração. Sejam $p \in M$ e $\eta \in\left(T_{p} M\right)^{\perp},|\eta|=1$. Seja $\left\{e_{1}, \ldots, e_{n}\right\}$ uma base ortonormal de $T_{p} M$, para a qual $S_{\eta}$ é diagonal, isto é, $S_{\eta}\left(e_{i}\right)=\lambda_{i} e_{i}$, onde $\lambda_{i}$ são os valores próprios de $S_{\eta}$.

Note que

$$
B\left(e_{i}, e_{j}\right)=H_{\eta}\left(e_{i}, e_{j}\right) \eta=\left\langle S_{\eta}\left(e_{i}\right), e_{j}\right\rangle \eta=\lambda_{i} \delta_{i j} \eta
$$

Portanto (1.3) se escreve

$$
K\left(e_{i}, e_{j}\right)-\bar{K}\left(e_{i}, e_{j}\right)=\lambda_{i} \lambda_{j}
$$

Definição 1.4.5. Se $M$ é uma hipersuperfície, definimos a curvatura de Gauss-Kronecker de $M$ por $K=\operatorname{det} S_{\eta}=k_{1} \cdot \ldots \cdot k_{n}$ e a curvatura média de $M$ por $H=\frac{1}{n} \operatorname{tr} S_{\eta}=\frac{k_{1}+\ldots+k_{n}}{n}$.

Teorema 1.4.1 (Egregium de Gauss). Se $M^{2}$ é uma hipersuperfície em $\mathbb{R}^{3}$, então para qualquer $p \in M$ e para quaisquer vetores linearmente independentes $X, Y$ de $T_{p} M, K(p)=K(X, Y)$. Portanto, a curvatura de Gauss é um invariante isométrico de $(M, g)$.

Proposição 1.4.9. Seja $f: M \longrightarrow \bar{M}$ uma imersão isométrica de codimensão igual a 1. Sejam $X, Y, Z$ campos tangentes e $\eta$ um campo normal unitário a $M$. Então as seguintes equaçôes se verificam:

1) Equação de Gauss

$$
R(X, Y) Z-\bar{R}(X, Y) Z=\langle S X, Z\rangle S Y-\langle S Y, Z\rangle S X
$$

2) Equação de Codazzi

$$
\bar{R}(X, Y) N=\nabla_{X} S Y-\nabla_{Y} S X-S([X, Y]) .
$$

Quando $\bar{M}$ tem curvatura seccional constante, a equação acima se reduz a

$$
\nabla_{X}(S Y)-\nabla_{Y}(S X)=S([X, Y])
$$

Demonstração. Primeiramente mostraremos a equação de Codazzi. Observe que $\bar{\nabla}_{X} \eta=\left(\bar{\nabla}_{X} \eta\right)^{T}+$ $\left(\bar{\nabla}_{X} \eta\right)^{N}=-S_{\eta}(X)+\nabla_{X}^{\frac{1}{X}} \eta$, isto é, $\nabla_{X}^{\frac{1}{X}} \eta=\bar{\nabla}_{X} \eta+S_{\eta}(X)$. Seja $\eta(q) \in(T M)^{\perp}$ tal que $\langle\eta, \eta\rangle=1$. Então $X\langle\eta, \eta\rangle=0$. Se $X \in T M$, segue que $\left\langle\nabla_{X} \eta, \eta\right\rangle=0$, daí temos $\nabla_{X} \eta \in T M$. Consequentemente $\nabla_{X}^{\perp} \eta=0$. 
Sabemos que se o espaço ambiente $\bar{M}$ tem curvatura constante, a equação de Codazzi se reduz a

$$
\left(\bar{\nabla}_{Y} B\right)(X, Z, \eta)=\left(\bar{\nabla}_{X} B\right)(Y, Z, \eta) .
$$

Mas note que

$$
\begin{aligned}
\left(\bar{\nabla}_{X} B\right)(Y, Z, \eta) & =X B(Y, Z, \eta)-B\left(\nabla_{X} Y, Z, \eta\right)-B\left(Y, \nabla_{X} Z, \eta\right) \\
& =X\langle B(Y, Z), \eta\rangle-\left\langle B\left(\nabla_{X} Y, Z\right), \eta\right\rangle-\left\langle B\left(Y, \nabla_{X} Z\right), \eta\right\rangle \\
& =X\left\langle S_{\eta} Y, Z\right\rangle-\left\langle S_{\eta}\left(\nabla_{X} Y\right), Z\right\rangle-\left\langle S_{\eta} Y, \nabla_{X} Z\right\rangle \\
& =\left\langle\nabla_{X}\left(S_{\eta} Y\right), Z\right\rangle-\left\langle S_{\eta}\left(\nabla_{X} Y\right), Z\right\rangle .
\end{aligned}
$$

De maneira análoga,

$$
\left(\bar{\nabla}_{Y} B\right)(X, Z, \eta)=\left\langle\nabla_{Y}\left(S_{\eta} X\right), Z\right\rangle-\left\langle S_{\eta}\left(\nabla_{Y} X\right), Z\right\rangle
$$

Logo, $\nabla_{X}\left(S_{\eta} Y\right)-S_{\eta}\left(\nabla_{X} Y\right)-\nabla_{Y}\left(S_{\eta} Y\right)+S_{\eta}\left(\nabla_{Y} X\right)=0$, isto é,

$$
\nabla_{X}\left(S_{\eta} Y\right)-\nabla_{Y}\left(S_{\eta} X\right)-S_{\eta}([X, Y])=0
$$

Mostraremos $\bar{R}(X, Y) N=\nabla_{X} S(Y)-\nabla_{Y} S(X)-S([X, Y])$.

Temos que

$$
\begin{aligned}
\bar{R}(X, Y) \eta & =\bar{\nabla}_{Y} \bar{\nabla}_{X} \eta-\bar{\nabla}_{X} \bar{\nabla}_{Y} \eta+\bar{\nabla}_{[X, Y]} \eta \\
& =\bar{\nabla}_{Y}\left(\nabla_{X}^{\perp} \eta-S_{\eta} X\right)-\bar{\nabla}_{X}\left(\nabla_{Y}^{\perp} \eta-S_{\eta} Y\right)+\nabla_{[X, Y]}^{\perp} \eta-S_{\eta}([X, Y]) \\
& =\bar{\nabla}_{X}\left(S_{\eta} Y\right)-\bar{\nabla}_{Y}\left(S_{\eta} X\right)-S_{\eta}([X, Y]) .
\end{aligned}
$$

Notemos que

$$
\begin{aligned}
\left\langle\bar{\nabla}_{X} S Y-\bar{\nabla}_{Y} S X, N\right\rangle & =\left\langle\bar{\nabla}_{X} S Y, N\right\rangle-\left\langle\bar{\nabla}_{Y} S X, N\right\rangle \\
& =X\langle S Y, N\rangle-\left\langle S Y, \bar{\nabla}_{X} N\right\rangle-Y\langle S X, N\rangle+\left\langle S X, \bar{\nabla}_{Y} N\right\rangle
\end{aligned}
$$

Como

$$
\begin{aligned}
\langle S Y, N\rangle & =\langle S X, N\rangle=0 \\
\left\langle S Y, \bar{\nabla}_{X} N\right\rangle & =\left\langle S Y,-S_{\eta} X\right\rangle \\
\left\langle S X, \bar{\nabla}_{Y} N\right\rangle & =\left\langle S X,-S_{\eta} Y\right\rangle,
\end{aligned}
$$


temos

$$
\begin{aligned}
\left\langle\bar{\nabla}_{X} S Y-\bar{\nabla}_{Y} S X, N\right\rangle & =-\langle S Y,-S X\rangle+\langle S X,-S Y\rangle \\
& =\langle S Y, S X\rangle-\langle S X, S Y\rangle \\
& =0 .
\end{aligned}
$$

Isto é, o campo $\bar{\nabla}_{X} S Y-\bar{\nabla}_{Y} S X$ é um campo tangente a $M$. Logo, podemos trocar $\bar{\nabla}_{X} S Y-$ $\bar{\nabla}_{Y} S X$ por $\nabla_{X} S Y-\nabla_{Y} S X$ na equação (1.4), obtendo

$$
\bar{R}(X, Y) \eta=\nabla_{X} S_{\eta}(Y)-\nabla_{Y} S_{\eta}(X)-S_{\eta}([X, Y])
$$

Vejamos agora como fica a equação de Gauss.

Sejam $X, Y, Z, T \in \mathfrak{X}(\bar{M})$ e $\mathrm{N} \in \mathfrak{X}(M)^{\perp}$ um campo normal unitário. Se a codimensão for igual a 1 , então

$$
B(X, T)=\lambda N,
$$

donde

$$
\lambda=\langle B(X, T), N\rangle=\langle S X, T\rangle .
$$

Logo

$$
B(X, T)=\langle S X, T\rangle N
$$

Segue daí que a equação de Gauss pode ser reescrita como

$$
\langle R(X, Y) Z-\bar{R}(X, Y) Z, T\rangle=\langle\langle S Y, T\rangle N,\langle S X, Z\rangle N\rangle-\langle\langle S X, T\rangle N,\langle S Y, Z\rangle N\rangle
$$

isto é,

$$
\langle R(X, Y) Z-\bar{R}(X, Y) Z, T\rangle=\langle\langle S X, Z\rangle S Y-\langle S Y, Z\rangle S X, T\rangle .
$$

Note que a igualdade anterior é válida para qualquer $T \in \mathfrak{X}(\bar{M})$ e, em particular, substituindo $T$ por $N$, obtemos

$$
\langle R(X, Y) Z-\bar{R}(X, Y) Z, N\rangle=0,
$$

ou seja $R(X, Y) Z-\bar{R}(X, Y) Z \in \mathfrak{X}(M)$. Portanto,

$$
R(X, Y) Z-\bar{R}(X, Y) Z=\langle S X, Z\rangle S Y-\langle S Y, Z\rangle S X .
$$




\subsection{Pares de Codazzi}

Nesta seção vamos estudar uma classe especial de pares fundamentais em uma superfície $\Sigma$, chamado pares de Codazzi.

Definição 1.5.1. Dizemos que um par fundamental $(\langle\rangle, S$,$) em \Sigma$ é um par de Codazzi se

$$
T_{S}(X, Y)=0, \quad X, Y \in \mathfrak{X}(\Sigma)
$$

e $S$ é dito Codazzi com respeito a $\langle$,$\rangle .$

Equivalentemente, $(I, I I) \in \mathcal{P}(\Sigma)$ é um par de Codazzi se, para qualquer parametrização local $(x, y)$, onde

$$
\begin{aligned}
I & =E d x^{2}+2 F d x d y+G d y^{2} \\
I I & =e d x^{2}+2 f d x d y+g d y^{2}
\end{aligned}
$$

o par $(I, I I)$ satisfaz às equações de Codazzi para superfícies em um espaço tridimensional. Isto $\dot{e}$,

$$
\begin{aligned}
& e_{y}-f_{x}-e \Gamma_{12}^{1}-f\left(\Gamma_{12}^{2}+\Gamma_{11}^{1}\right)+g \Gamma_{12}^{2}=0 \\
& f_{y}-g_{x}-e \Gamma_{22}^{1}-f\left(\Gamma_{22}^{2}+\Gamma_{12}^{1}\right)+g \Gamma_{12}^{2}=0
\end{aligned}
$$

onde $\Gamma_{i j}^{k}$ são os símbolos de Christoffel para a métrica Riemanniana I com relação à conexão Riemanniana de I. Estas equações são conhecidas como as equações de Codazzi.

Observação 1.5.1. Se $M^{2} \subset \bar{M}^{3}$ tal que $\bar{M}$ tem curvatura constante, então $(I, I I)$ é um par de Codazzi. De fato, como $\bar{M}$ tem curvatura constante, então segue do item (2) da Proposição 1.4 .9 que

$$
\nabla_{X}(S Y)-\nabla_{Y}(S X)=S([X, Y])
$$

ou seja,

$$
T_{S}(X, Y)=\nabla_{X} S Y-\nabla_{Y} S X-S[X, Y]=0 ; \quad X, Y \in \mathfrak{X}(M) .
$$

De um modo geral, se uma superfície é imersa em uma forma espacial n-dimensional (semiRiemanniana) e possui um campo normal $\eta$, unitário e paralelo, então a métrica induzida e sua segunda forma fundamental associada com $\eta$ constituem um par de Codazzi, isto é, se $M^{2} \subset \bar{M}^{n}$ com $\nabla^{\perp} \eta=0$, então $\left(I, I I_{\eta}\right)$ é par de Codazzi.

O conjunto de pares de Codazzi, denotado por $\mathcal{C}(\Sigma)$, é o subconjunto de $\mathcal{P}(\Sigma)$ composto pelos pares fundamentais cujo operador de forma associado é Codazzi. Assim, podemos pensar no tensor Codazzi como um tensor que mede o quão longe é um par fundamental de ser um par Codazzi. 
Antes de passarmos para o próximo resultado, lembremos das superfícies paralelas em $\mathbb{R}^{3}$.

Definição 1.5.2. Seja $X: U \longrightarrow \mathbb{R}^{3}$ uma superfície parametrizada regular. Então uma superfície paralela a $X$ à uma distancia a é uma superfície parametrizada

$$
Y(u, v)=X(u, v)+\boldsymbol{a} N(u, v)
$$

onde $N$ é a normal de Gauss.

Observação 1.5.2 (Ver [8]). Considerando uma superfície paralela $Y=X+\boldsymbol{a} N$, temos:

(i) As curvaturas principais de $Y$ são dadas por

$$
\overline{k_{i}}=\frac{k_{i}}{1-\boldsymbol{a} k_{i}}
$$

para $i=1,2$, onde $k_{1}$ e $k_{2}$ são as curvaturas principais de $X$.

(ii) A curvatura Gaussiana $\bar{K}$ e a curvatura Média $\bar{H}$ de $Y$ são dadas por

$$
\bar{K}=\frac{K}{1-2 \boldsymbol{a} H+\boldsymbol{a}^{2} K} \quad e \quad \bar{H}=\frac{H-K \boldsymbol{a}}{1-2 a H+\boldsymbol{a}^{2} K},
$$

onde $K$ e $H$ são as curvaturas Gaussiana e Média de X, respectivamente.

Observação 1.5.3. Considere o par fundamental $(I, I I)$. Definimos a terceira forma fundamental por $I I I(X, Y)=\langle S X, S Y\rangle$, para todo $X, Y \in \mathfrak{X}(\Sigma)$, onde $S$ é o operador forma de $\Sigma$.

Inspirado na teoria de superfícies paralelas, a proposição a seguir será importante para demonstrar o resultado principal do próximo capítulo para pares de Codazzi em superfícies completas com curvatura não-positiva.

Proposição 1.5.1. Sejam $(I, I I)$ um par de Codazzi sobre a superfície $\Sigma$ e III a terceira forma fundamental associada. Seja $a \in \mathbb{R}-\{0\}$ tal que $a k_{1} \neq 1$ e $a k_{2} \neq 1$. Considere a forma quadrática

$$
\Lambda_{a}=I-2 a I I+a^{2} I I I
$$

Então $\Lambda_{a}$ é métrica Riemanniana e sua curvatura é calculada como

$$
K\left(\Lambda_{a}\right)=\frac{K(I)}{\left(1-a k_{1}\right)\left(1-a k_{2}\right)} .
$$

Demonstração. Considere a terceira forma fundamental $I I I$ associada ao par $(I, I I)$. Sejam $(I, I I) \in \mathcal{P}(\Sigma), \mathrm{p} \in \Sigma^{\prime}$, sendo $\Sigma^{\prime}$ conjunto formado pelo interior do conjunto de pontos umbílicos e o conjunto de pontos não umbílicos e $(u, v)$ parâmetros locais duplamente ortogonais $(F=f=0)$. 
Temos:

$$
\begin{aligned}
I & =E d u^{2}+G d v^{2} \\
I I & =k_{1} E d u^{2}+k_{2} G d v^{2} \\
I I I & =k_{1}^{2} E d u^{2}+k_{2}^{2} G d v^{2} .
\end{aligned}
$$

Considerando uma base ortonormal $\left\{e_{1}, e_{2}\right\}$ de $T_{p_{0}} \Sigma$, tal que $p_{0} \in \Sigma$ é um ponto fixo, temos que

$$
\Lambda_{a} \equiv\left(\begin{array}{cc}
\left(1-a k_{1}\right)^{2} & 0 \\
0 & \left(1-a k_{2}\right)^{2}
\end{array}\right) .
$$

Então

$$
\Lambda_{a}\left(e_{i}, e_{j}\right)=\left(1-a k_{i}\right)^{2} \delta_{i j},
$$

o que prova que a forma quadrática $\Lambda_{a}$ é positiva definida e, portanto, é a métrica Riemanniana sobre a superfície. Agora note que de (1.6) e (1.7)

$$
\Lambda_{a}=\left(1-a k_{1}\right)^{2} E d u^{2}+\left(1-a k_{2}\right)^{2} G d v^{2} .
$$

Denotando

$$
\begin{aligned}
E_{a} & =\left(1-a k_{1}\right)^{2} E \\
F_{a} & =0 \\
G_{a} & =\left(1-a k_{2}\right)^{2} G,
\end{aligned}
$$

temos os coeficientes da primeira forma fundamental da formula quadrática $\Lambda_{a}$. Usando a equação de Gauss para curvatura em parâmetros ortogonais temos

$$
K\left(\Lambda_{a}\right)=-\frac{1}{2 \sqrt{E_{a} G_{a}}}\left\{\left(\frac{\left(E_{a}\right)_{v}}{\sqrt{E_{a} G_{a}}}\right)_{v}+\left(\frac{\left(G_{a}\right)_{u}}{\sqrt{E_{a} G_{a}}}\right)_{u}\right\} .
$$

Segue da parametrização duplamente ortogonal que as curvas coordenadas são linhas de curvatura, logo as equações de Mainardi-Codazzi assumem a seguinte forma:

$$
e_{v}=\frac{E_{v}}{2}\left(\frac{e}{E}+\frac{g}{G}\right) \quad \text { e } \quad g_{u}=\frac{G_{u}}{2}\left(\frac{e}{E}+\frac{g}{G}\right) .
$$

e

Por outro lado, $e=k_{1} E$ e $g=k_{2} G$. Então

$$
\begin{aligned}
\left(k_{1} E\right)_{v} & =\frac{E_{v}}{2}\left(\frac{e}{E}+\frac{g}{G}\right) \\
\left(k_{2} G\right)_{u} & =\frac{G_{u}}{2}\left(\frac{e}{E}+\frac{g}{G}\right) .
\end{aligned}
$$


De (1.10), (1.11), $k_{1}=\frac{e}{E}$ e $k_{2}=\frac{g}{G}$ temos

$$
\begin{aligned}
\left(k_{1}\right)_{v} & =\frac{E_{v}}{2 E}\left(k_{2}-k_{1}\right) \\
\left(k_{2}\right)_{u} & =\frac{G_{u}}{2 G}\left(k_{1}-k_{2}\right) .
\end{aligned}
$$

Substituindo (1.8) em (1.9) obtemos

$$
\begin{aligned}
K\left(\Lambda_{a}\right)= & -\frac{1}{2\left(1-a k_{1}\right)\left(1-a k_{2}\right) \sqrt{E G}} . \\
& {\left[\left(\frac{-2 a\left(k_{1}\right)_{v} E+\left(1-a k_{1} E_{v}\right)}{\left(1-a k_{2}\right) \sqrt{E G}}\right)_{v}+\left(\frac{-2 a\left(k_{2}\right)_{u} G-\left(1-a k_{2} G_{u}\right)}{\left(1-a k_{1}\right) \sqrt{E G}}\right)_{u}\right] . }
\end{aligned}
$$

Agora substituindo (1.12) na equação acima temos que:

$$
K\left(\Lambda_{a}\right)=-\frac{1}{\left(1-a k_{1}\right)\left(1-a k_{2}\right) 2 \sqrt{E G}}\left[\left(\frac{E_{v}}{\sqrt{E G}}\right)_{v}+\left(\frac{G_{u}}{\sqrt{E G}}\right)_{u}\right] .
$$

Portanto,

$$
K\left(\Lambda_{a}\right)=\frac{K(I)}{\left(1-a k_{1}\right)\left(1-a k_{2}\right)} .
$$

Observação 1.5.4. Se $a \in \mathbb{R} \backslash\{0\}$ satisfaz $a k_{1} \neq 1$ e a $k_{2} \neq 1$ em $\Sigma$, então de (1.6) e considerando uma superfície paralela $Y=X+a N$ temos a seguinte forma quadrática:

$$
\begin{aligned}
\Lambda_{a} & =\langle d Y, d Y\rangle \\
& =\langle d X+a d N, d X+a d N\rangle \\
& =\langle d X, d X\rangle+2 a\langle d X, d N\rangle+a^{2}\langle d N, d N\rangle \\
& =I-2 a I I+a^{2} I I I .
\end{aligned}
$$

\subsection{Superfície de Riemann e Forma Quadrática Holomorfa}

Uma ferramenta que usaremos com grande frequência é Análise Complexa. Abordaremos brevemente como introduzir uma variável complexa em uma superfície e o fato que induz em uma superfície orientável uma estrutura de superfície de Riemann. Veremos também forma quadrática holomorfa e obteremos as representações de pares fundamentais em uma superfície com respeito a um parâmetro especial: o parâmetro isotérmico. 
De agora em diante, passaremos a identificar o plano complexo $\mathbb{C}$ com o plano $\mathbb{R}^{2}$, fazendo $z=x+i y$, onde $(x, y) \in \mathbb{R}^{2}$ e $z \in \mathbb{C}$.

Seja $\psi: U \subset \mathbb{C} \longrightarrow \mathbb{C}$ uma função. Podemos escrever $\psi$ como $\psi(z)=u(x, y)+i v(x, y)$, onde $u, v: U \longrightarrow \mathbb{R}$ são funções reais.

Definição 1.6.1. Suponha que $U$ é aberto em $\mathbb{C}$. Dizemos que $\psi$ é holomorfa se as funções reais u e $v$ possuem derivadas parciais contínuas que satisfazem às equações abaixo:

$$
\frac{\partial u}{\partial x}=\frac{\partial v}{\partial y} \quad e \quad \frac{\partial u}{\partial y}=-\frac{\partial v}{\partial x} .
$$

As equações acima são conhecidas como as equações de Cauchy-Riemann.

Definição 1.6.2. Uma superfície de Riemann $M$ é uma variedade 2-dimensional, conexa de Hausdorff com estrutura diferenciável $\left\{\left(U_{\alpha}, X_{\alpha}\right)\right\}$, tal que $\left\{X_{\alpha}\left(U_{\alpha}\right)\right\}$ é uma cobertura aberta de $M$, onde $X_{\alpha}: U_{\alpha} \in \mathbb{C} \longrightarrow M$ é um homeomorfismo de um subconjunto aberto do plano complexo $\mathbb{C}$ de modo que s mudança de parâmetros

$$
f_{\alpha \beta}=X_{\alpha}^{-1} \circ X_{\beta}: X_{\beta}^{-1}\left(X_{\beta}\left(U_{\beta}\right) \cap X_{\alpha}\left(U_{\alpha}\right)\right) \longrightarrow X_{\beta}\left(U_{\beta}\right) \cap X_{\alpha}\left(U_{\alpha}\right)
$$

são holomorfas sempre que $\left(X_{\beta}\left(U_{\beta}\right) \cap X_{\alpha}\left(U_{\alpha}\right)\right) \neq \emptyset$.

Teorema 1.6.1 (Ver [19]). Toda superfície de Riemann é orientável.

Definição 1.6.3. Seja $X: U \longrightarrow \mathbb{C}$ um difeomorfismo. Dizemos que $X$ um é biholomorfismo ou um difeomorfismo holomorfo se $X$ e $X^{-1}$ são holomorfas.

Teorema 1.6.2 (Teorema da Uniformização de Riemann). O recobrimento universal de qualquer superfície de Riemann é conformemente equivalente (difeomorfismo holomorfo) ou a um disco ou ao plano ou à esfera.

Definição 1.6.4. Uma forma quadrática $Q=F d z^{2}$, onde $F: U \longrightarrow \mathbb{C}$ é uma função contínua, é dita holomorfa se $F$ é uma função holomorfa.

Teorema 1.6.3. (Liouville) Seja $f: \mathbb{C} \longrightarrow \mathbb{C}$ uma função inteira (analítica sobre todo $\mathbb{C}$ ). Então é limitada se, e somente se, $f$ é constante.

Teorema 1.6.4. Em uma superfície $\Sigma$, compacta de gênero 0 , não existe diferencial quadrática holomorfa $\mathcal{Q} \in \mathcal{Q}_{\mathbb{C}}(\Sigma)$, exceto a trivial, isto é, $\mathcal{Q}=0$.

Demonstração. Se $\Sigma$ é uma superfície compacta de gênero zero, então pelo teorema da uniformização de Riemann, $\Sigma$ é conformemente equivalente (difeomorfismo holomorfo) à esfera de Riemann $\mathbb{C} \cup\{\infty\}$. 
Considerando as parametrizações locais

$$
\psi: \mathbb{S}^{2} \backslash\{N\} \longrightarrow \mathbb{C}
$$

e

$$
\phi: \mathbb{S}^{2} \backslash\{N\} \longrightarrow \mathbb{C}
$$

onde $\psi$ e $\phi$ são as projeções estereográficas.

Denotaremos por $z$ e $w$ os parâmetros isotérmicos sobre as parametrizações locais $\left(\mathbb{S}^{2} \backslash N, \psi\right)$ e $\left(\mathbb{S}^{2} \backslash N, \phi\right)$ respectivamente. Sabemos que $w=\frac{1}{z}$.

Com respeito às parametrizações locais $\psi$ e $\phi$ podemos escrever $\mathcal{Q}(z)=Q(z) d z^{2}$ e $\mathcal{Q}(w)=$ $\bar{Q}(z) d w^{2}$, respectivamente, onde $Q(z)$ e $Q(w)$ são formas quadráticas holomorfas.

$\mathrm{Na}$ interseção $\mathbb{C} \backslash\{0\}$ temos que

$$
\mathcal{Q}(w)=\bar{Q}(w) d w^{2}=\bar{Q}\left(\frac{1}{z}\right)\left(d\left(\frac{1}{z}\right)\right)^{2}=\bar{Q}\left(\frac{1}{z}\right) z^{-4} d z^{2}=w^{4} \bar{Q}(w) d z^{2}
$$

e então

$$
Q(z)=w^{4} \bar{Q}(w)
$$

Assim

$$
\lim _{z \rightarrow \infty} Q(z)=\lim _{w \rightarrow 0} w^{4} \bar{Q}(w)=0 . \bar{Q}(0)=0
$$

Logo $Q(z)$ e $\bar{Q}(w)$ são funções limitadas e inteiras. Desse modo, pelo Teorema de Liouville temos $Q(z)$ e $\bar{Q}(w)$ são constante, ou seja, $Q(z)=a$ e $\bar{Q}(w)=b$. Então $a=b w^{4}$, para todo $w \in \mathbb{C}$. Segue que $b=a=0$. Portanto $\mathcal{Q} \equiv 0$

A seguir, apresentaremos um resultado que caracteriza as funções holomorfas definidas em uma superfície de Riemann.

Proposição 1.6.1. Seja $\psi: M \longrightarrow \mathbb{C}$ uma função. Então $\psi$ é holomorfa se, e somente se, $\frac{\partial \psi}{\partial \bar{z}}=0$, para todas parametrizaçôes locais de $M$. 
Demonstração. Podemos escrever $\psi$ como $\psi(z)=u(z)+i v(z)$, tais que $u, v \in C^{\infty}(M)$. Então

$$
\begin{aligned}
\frac{\partial \psi}{\partial \bar{z}} & =\frac{1}{2}\left(\frac{\partial \psi}{\partial \bar{z}}+i \frac{\partial \psi}{\partial \bar{z}}\right) \\
& =\frac{1}{2}\left[\frac{\partial u}{\partial x}+i \frac{\partial v}{\partial y}+i\left(\frac{\partial u}{\partial y}+i \frac{\partial v}{\partial y}\right)\right] \\
& =\frac{1}{2}\left[\frac{\partial u}{\partial x}+i \frac{\partial v}{\partial x}+i \frac{\partial u}{\partial y}-\frac{\partial v}{\partial y}\right] \\
& =\frac{1}{2}\left[\frac{\partial u}{\partial x}-\frac{\partial v}{\partial y}+i\left(\frac{\partial v}{\partial x}+\frac{\partial u}{\partial y}\right)\right]
\end{aligned}
$$

Logo $\frac{\partial \psi}{\partial \bar{z}}=0$ é equivalente a

$$
\frac{\partial u}{\partial x}=\frac{\partial v}{\partial y} \quad e \quad \frac{\partial v}{\partial x}=-\frac{\partial u}{\partial y}
$$

que são as condições de Cauchy-Riemann.

Definição 1.6.5. Um domínio $U \in \mathbb{C}$ é chamado simplesmente conexo se todas curvas fechadas em $U$ pode ser deformada continuamente em um ponto sem sair de $U$.

Sejam $\Sigma$ uma superfícies orientável. Introduzindo uma variável complexa $z=x+i y$ e $\bar{z}=x-i y$ seu conjugado, podemos escrever

$$
x=\frac{z+\bar{z}}{2} \quad e \quad y=\frac{z-\bar{z}}{2 i} .
$$

Além disso, podemos definir as 1 -formas complexas locais $d z=d x+i d y$ e $d \bar{z}=d x-i d y$ que formam a base dual $\left\{\partial_{z}, \partial_{\bar{z}}\right\}$. Assim trabalharemos indistintamente com um parâmetro $(x, y)$ complexo $z=x+i y$.

Portanto, podemos reescrever o par fundamental $(I, I I)$ em $\Sigma$ como:

$$
\begin{aligned}
I & =p d z^{2}+2 \lambda|d z|^{2}+\bar{p} d \bar{z}^{2} \\
I I & =Q d z^{2}+2 \rho|d z|^{2}+\bar{Q} d \bar{z}^{2}
\end{aligned}
$$

em que 


$$
\begin{aligned}
p & =I\left(\partial_{z}, \partial_{z}\right)=\frac{1}{4}(E-G-2 i F) \\
\bar{p} & =I\left(\partial_{\bar{z}}, \partial_{\bar{z}}\right)=\frac{1}{4}(E-G+2 i F) \\
\lambda & =I\left(\partial_{z}, \partial_{\bar{z}}\right)=\frac{1}{2}(E+G) \\
Q & =I\left(S \partial_{z}, \partial_{z}\right)=\frac{1}{4}(e-g-2 i f) \\
\bar{Q} & =I\left(S \partial_{\bar{z}}, \partial_{\bar{z}}\right)=\frac{1}{4}(e-g+2 i f) \\
\rho & =I\left(S \partial_{z}, \partial_{\bar{z}}\right)=\frac{1}{2}(e+g) \\
H & =H(I, I I)=\frac{p \bar{Q}-2 \lambda \rho+\bar{p} Q}{2\left(|p|^{2}-\lambda^{2}\right)} \\
K & =K(I, I I)=\frac{|Q|^{2}-\rho^{2}}{|p|^{2}-\lambda^{2}}
\end{aligned}
$$

Podemos, ainda, expressar os coeficientes da conexão $\nabla$ associados a z e $\bar{z}$ como

$$
\begin{aligned}
\nabla_{\partial_{z}} \partial_{z} & ={ }^{\mathbb{C}} \Gamma_{11}^{1} \partial_{z}+{ }^{\mathbb{C}} \Gamma_{11}^{2} \partial_{\bar{z}} \\
\nabla_{\partial_{z}} \partial_{\bar{z}} & ={ }^{\mathbb{C}} \Gamma_{12}^{1} \partial_{z}+{ }^{\mathbb{C}} \Gamma_{12}^{2} \partial_{\bar{z}} \\
\nabla_{\partial_{\bar{z}}} \partial_{\bar{z}} & ={ }^{\mathbb{C}} \Gamma_{22}^{1} \partial_{z}+{ }^{\mathbb{C}} \Gamma_{22}^{2} \partial_{\bar{z}}
\end{aligned}
$$

sendo ${ }^{\mathbb{C}} \Gamma_{i j}^{k}$ os símbolos de Christoffel da conexão associados aos parâmetros $(z, \bar{z})$. Vejamos como relacionam os símbolos de Christoffel $\Gamma_{i j}^{k}$ associados aos parâmetros $(x, y)$ com os símbolos $\mathbb{C}^{k} \Gamma_{i j}^{k}$ associados aos parâmetros $(z, \bar{z})$ onde $z=x+i y$.

$$
\begin{array}{r}
\nabla_{\partial_{z}} \partial_{z}=\frac{1}{4} \nabla_{\partial_{x}-i \partial_{y}}\left(\partial_{x}-i \partial_{y}\right)=\frac{1}{4}\left(\nabla_{\partial_{x}} \partial_{x}-2 i \nabla_{\partial_{x}} \partial_{y}-\nabla_{\partial_{y}} \partial_{y}\right) \\
=\frac{1}{4}\left(\left(\Gamma_{11}^{1}-2 i \Gamma_{12}^{1}-\Gamma_{22}^{1}\right) \partial_{x}+\left(\Gamma_{11}^{2}-2 i \Gamma_{12}^{2}-\Gamma_{22}^{2}\right) \partial_{y}\right) \\
=\frac{1}{4}\left(\Gamma_{11}^{1}-\Gamma_{22}^{1}+2 \Gamma_{12}^{2}+i\left(\Gamma_{11}^{2}-\Gamma_{22}^{2}-2 \Gamma_{12}^{1}\right)\right) \partial_{z} \\
+\frac{1}{4}\left(\Gamma_{11}^{1}-\Gamma_{22}^{1}-2 \Gamma_{12}^{2}-i\left(\Gamma_{11}^{2}-\Gamma_{22}^{2}-2 \Gamma_{12}^{1}\right)\right) \partial_{\bar{z}}
\end{array}
$$

Logo,

$$
\begin{aligned}
& \mathbb{C}_{\Gamma_{11}^{1}}^{1}=\frac{1}{4}\left(\Gamma_{11}^{1}-\Gamma_{22}^{1}+2 \Gamma_{12}^{2}+i\left(\Gamma_{11}^{2}-\Gamma_{22}^{2}-2 \Gamma_{12}^{1}\right)\right) \partial_{z} \\
& { }^{\mathbb{C}} \Gamma_{11}^{2}=\frac{1}{4}\left(\Gamma_{11}^{1}-\Gamma_{22}^{1}-2 \Gamma_{12}^{2}-i\left(\Gamma_{11}^{2}-\Gamma_{22}^{2}-2 \Gamma_{12}^{1}\right)\right) \partial_{\bar{z}}
\end{aligned}
$$


De forma análoga,

$$
\begin{aligned}
{ }^{\mathbb{C}} \Gamma_{12}^{1} & =\frac{1}{4}\left[\Gamma_{11}^{1}+\Gamma_{22}^{1}+i\left(\Gamma_{11}^{2}+\Gamma_{22}^{2}\right)\right] \\
{ }^{C} \Gamma_{12}^{2} & =\frac{1}{4}\left[\Gamma_{11}^{1}+\Gamma_{22}^{1}-i\left(\Gamma_{11}^{2}+\Gamma_{22}^{2}\right)\right] \\
{ }^{C} \Gamma_{22}^{1} & =\frac{1}{4}\left[\Gamma_{11}^{1}-\Gamma_{22}^{1}-2 \Gamma_{12}^{2}+i\left(\Gamma_{11}^{2}-\Gamma_{22}^{2}+2 \Gamma_{12}^{1}\right)\right] \\
{ }^{C} \Gamma_{22}^{2} & =\frac{1}{4}\left[\Gamma_{11}^{1}-\Gamma_{22}^{1}+2 \Gamma_{12}^{2}-i\left(\Gamma_{11}^{2}-\Gamma_{22}^{2}-2 \Gamma_{12}^{1}\right)\right]
\end{aligned}
$$

Usando (1.23) e (1.24) estabelecemos as seguintes relações

$$
\begin{aligned}
& { }^{{ }^{C}} \Gamma_{11}^{1}={ }^{\mathbb{C}} \bar{\Gamma}_{22}^{2} \\
& { }^{\mathbb{C}} \Gamma_{11}^{2}={ }^{\mathbb{C}} \bar{\Gamma}_{22}^{1} \\
& { }^{{ }^{C}} \Gamma_{12}^{1}={ }^{\mathbb{C}} \bar{\Gamma}_{12}^{2} .
\end{aligned}
$$

Além disso, substituindo as equações (1.14) e (1.16) nos símbolos de Christoffel (1.2), obtemos as seguintes relações,

$$
\begin{aligned}
{ }^{C} \Gamma_{11}^{1} & =\frac{1}{2\left(|p|^{2}-\lambda^{2}\right)}\left(\bar{p} p_{z}-2 \lambda \lambda_{z}+\lambda p_{\bar{z}}\right) \\
{ }^{C} \Gamma_{11}^{2} & =-\frac{1}{2\left(|p|^{2}-\lambda^{2}\right)}\left(p p_{\bar{z}}-2 p \lambda_{z}+\lambda p_{\bar{z}}\right) \\
\mathbb{C}_{\Gamma_{12}^{1}} & =\frac{1}{2\left(|p|^{2}-\lambda^{2}\right)}\left(\bar{p} p_{z}-\lambda \bar{p}_{z}\right) \\
{ }^{C} \Gamma_{12}^{2} & =\frac{1}{2\left(|p|^{2}-\lambda^{2}\right)}\left(p \bar{p}_{z}-\lambda p_{\bar{z}}\right) \\
{ }^{C} \Gamma_{22}^{1} & =-\frac{1}{2\left(|p|^{2}-\lambda^{2}\right)}\left(\bar{p} \bar{p}_{z}-2 \bar{p} \lambda_{\bar{z}}+\lambda \bar{p}_{\bar{z}}\right) \\
\mathbb{C}_{\Gamma_{22}^{2}} & =\frac{1}{2\left(|p|^{2}-\lambda^{2}\right)}\left(p \bar{p}_{\bar{z}}-2 \lambda \lambda_{\bar{z}}+\lambda \bar{p}_{z}\right) .
\end{aligned}
$$

O índice $\mathbb{C}$ se refere ao parâmetro $(z, \bar{z})$ do qual prescindiremos sempre e quando não existir confusão.

Observação 1.6.1. Sabemos que para parâmetros locais quaisquer $(x, y)$ em que o par fundamental $(I, I I)$ vem dado por

$$
\begin{aligned}
I & =E d x^{2}+2 F d x d y+G d y^{2} \\
I I & =e d x^{2}+2 f d x d y+g d y^{2},
\end{aligned}
$$

se verificam as equações de Codazzi, isto é,

$$
\begin{aligned}
& \left\langle T_{S}\left(\partial_{x}, \partial_{y}\right), \partial_{y}\right\rangle=g_{x}-f_{y}+e \Gamma_{22}^{1}-g \Gamma_{12}^{2}+f\left(\Gamma_{22}^{2}-\Gamma_{12}^{1}\right) \\
& \left\langle T_{S}\left(\partial_{x}, \partial_{y}\right), \partial_{x}\right\rangle=f_{x}-e_{y}+e \Gamma_{12}^{1}-g \Gamma_{11}^{2}+f\left(\Gamma_{12}^{2}-\Gamma_{11}^{1}\right) .
\end{aligned}
$$


Podemos reescrever as equações anteriores usando um parâmetro local complexo $z=x+i y$, em que o par fundamental $(I, I I)$ vem dado por (1.13). Desta forma

$$
\begin{aligned}
\left\langle T_{S}\left(\partial_{z}, \partial_{\bar{z}}\right), \partial_{\bar{z}}\right\rangle & =\bar{Q}_{z}-\rho_{\bar{z}}+Q{ }^{\mathbb{C}} \Gamma_{22}^{1}+\rho\left({ }^{\mathbb{C}} \Gamma_{22}^{2}-{ }^{\mathbb{C}} \Gamma_{12}^{1}\right)-\bar{Q}{ }^{\mathbb{C}} \Gamma_{12}^{2} \\
\left\langle T_{S}\left(\partial_{z}, \partial_{\bar{z}}\right), \partial_{z}\right\rangle & =-Q_{\bar{z}}+\rho_{z}+Q{ }^{\mathbb{C}} \Gamma_{12}^{1}+\rho\left({ }^{\mathbb{C}} \Gamma_{12}^{2}-{ }^{\mathbb{C}} \Gamma_{11}^{1}\right)-\bar{Q}{ }^{\mathbb{C}} \Gamma_{11}^{2}
\end{aligned}
$$

Vamos escrever o Tensor de curvatura e a curvatura intrínseca em um sistema de coordenadas.

Assim, dada uma parametrização $(x, y)$, temos que

$$
\begin{aligned}
R\left(\partial_{x}, \partial_{y}\right) \partial_{x}= & \nabla_{\partial_{y}} \nabla_{\partial_{x}} \partial_{x}-\nabla_{\partial_{x}} \nabla_{\partial_{y}} \partial_{x} \\
= & \nabla_{\partial_{y}}\left(\Gamma_{11}^{1} \partial_{x}+\Gamma_{11}^{2} \partial_{y}\right)-\nabla_{\partial_{x}}\left(\Gamma_{12}^{1} \partial_{x}+\Gamma_{12}^{2} \partial_{y}\right) \\
= & \left(\left(\Gamma_{11}^{1}\right)_{y}-\left(\Gamma_{12}^{1}\right)_{x}\right) \partial_{x}+\left(\left(\Gamma_{11}^{2}\right)_{y}-\left(\Gamma_{12}^{2}\right)_{x}\right) \partial_{y} \\
& +\Gamma_{11}^{1} \nabla_{\partial_{x}} \partial_{y}+\Gamma_{11}^{2} \nabla_{\partial_{y}} \partial_{y}-\Gamma_{12}^{1} \nabla_{\partial_{x}} \partial_{x}-\Gamma_{12}^{2} \nabla_{\partial_{x}} \partial_{y} \\
= & \left(\left(\Gamma_{11}^{2}\right)_{y}-\left(\Gamma_{12}^{1}\right)_{x}+\Gamma_{11}^{2} \Gamma_{22}^{1}-\Gamma_{12}^{2} \Gamma_{12}^{1}\right) \partial_{x} \\
& \left(\left(\Gamma_{11}^{2}\right)_{y}-\left(\Gamma_{12}^{1}\right)_{x}+\Gamma_{11}^{1} \Gamma_{12}^{2}+\Gamma_{11}^{2} \Gamma_{22}^{2}-\Gamma_{12}^{1} \Gamma_{11}^{2}-\Gamma_{12}^{2} \Gamma_{12}^{2}\right) \partial_{x}
\end{aligned}
$$

Logo

$$
\begin{aligned}
K(I)= & \frac{F}{E G-F^{2}}\left(\left(\Gamma_{11}^{2}\right)_{y}-\left(\Gamma_{12}^{1}\right)_{x}+\Gamma_{11}^{2} \Gamma_{22}^{1}-\Gamma_{12}^{2} \Gamma_{12}^{1}\right) \\
& +\frac{G}{E G-F^{2}}\left(\left(\Gamma_{11}^{2}\right)_{y}-\left(\Gamma_{12}^{1}\right)_{x}+\Gamma_{11}^{1} \Gamma_{12}^{2}+\Gamma_{11}^{2} \Gamma_{22}^{2}-\Gamma_{12}^{1} \Gamma_{11}^{2}-\Gamma_{12}^{2} \Gamma_{12}^{2}\right) .
\end{aligned}
$$

Além disso, usando (1.14)-(1.19) e (1.25)-(1.33), de forma análoga, podemos observar que na parametrização $(z, \bar{z})$ temos:

$$
\begin{aligned}
& K(I)=\frac{\lambda}{|p|^{2}-\lambda^{2}}\left(\left({ }^{\mathbb{C}} \Gamma_{11}^{2}\right)_{y}-\left({ }^{\mathbb{C}} \Gamma_{12}^{1}\right)_{x}+{ }^{\mathbb{C}} \Gamma_{11}^{2}{ }^{\mathbb{C}} \Gamma_{22}^{1}-{ }^{\mathbb{C}} \Gamma_{12}^{2}{ }^{\mathbb{C}} \Gamma_{12}^{1}\right) \\
& +\frac{G}{E G-F^{2}}\left(\left({ }^{\mathbb{C}} \Gamma_{11}^{2}\right)_{y}-\left({ }^{\mathbb{C}} \Gamma_{12}^{1}\right)_{x}+{ }^{\mathbb{C}} \Gamma_{11}^{1}{ }^{\mathbb{C}} \Gamma_{12}^{2}+{ }^{\mathbb{C}} \Gamma_{11}^{2}{ }^{\mathbb{C}} \Gamma_{22}^{2}-{ }^{\mathbb{C}} \Gamma_{12}^{1}{ }^{\mathbb{C}} \Gamma_{11}^{2}-{ }^{\mathbb{C}} \Gamma_{12}^{2}{ }^{\mathbb{C}} \Gamma_{12}^{2}\right)
\end{aligned}
$$

Sejam $U \in \mathbb{C}$ um domínio simplesmente conexo e $X(u, v): U \longrightarrow \mathbb{R}^{3}$ uma superfície regular parametrizada $M$. Se $E=G=\lambda$ e $F=0$, então $X$ é uma aplicação conforme e os parâmetros $u$ e $v$ são chamados coordenadas isotérmicas para a superfície $M$.

Teorema 1.6.5 (Ver [30]). Seja $M$ uma superfície. Todo ponto regular de $M$ tem um vizinhança na qual existe uma reparametrização em termos de coordenadas isotérmicas.

Do teorema acima decorre que uma métrica Riemanniana em uma superfície orientável $M$ induz em $M$ uma estrutura de superfície de Riemann.

Descrevemos a seguir a representação de uma par fundamental $(I, I I)$ em termos de um parâmetro conforme local, assim como certas equações que serão úteis nas demonstrações de alguns 
resultados dos próximos capítulos.

Observação 1.6.2. Quando trabalharmos com parâmetros isotérmicos, sempre o faremos usando o parâmetro complexo associado $z=u+i v$.

Proposição 1.6.2. Sejam $(I, I I)$ um par fundamental em $M$ e z um parâmetro conforme local para a métrica Riemanniana I. Então

$$
\begin{aligned}
I & =2 \lambda|d z|^{2} \\
I I & =Q d z^{2}+2 \rho|d z|^{2}+\bar{Q} d \bar{z}^{2}
\end{aligned}
$$

Além disso, as seguintes equações se verificam:

$$
\begin{aligned}
K & =H^{2}-\frac{|Q|^{2}}{\lambda^{2}} \\
S \partial_{z} & =H \partial_{z}+\frac{Q \partial_{\bar{z}}}{\lambda} \\
\Gamma_{11}^{1} & =\frac{\lambda_{z}}{\lambda} \\
\Gamma_{11}^{2} & =\Gamma_{12}^{1}=0 \\
T_{S}\left(\partial_{z}, \partial_{\bar{z}}\right) & =T_{H}\left(\partial_{z}, \partial_{\bar{z}}\right)+\frac{1}{\lambda}\left(\bar{Q}_{z} \partial_{z}+Q_{\bar{z}} \partial_{\bar{z}}\right) \\
\left\langle T_{S}\left(\partial_{z}, \partial_{\bar{z}}\right), \partial_{z}\right\rangle & =\lambda H_{z}-Q_{\bar{z}} \\
K(I) & =-\frac{1}{\lambda}(\ln \lambda)_{z \bar{z}} .
\end{aligned}
$$

Demonstração. Se $z$ é parâmetro conforme para $I$, então $p=0$ e, portanto, de (1.20), temos que a curvatura média do par $(I, I I)$ é dada por $H=\frac{\rho}{\lambda}$. Então $\rho=\lambda H$ e, de (1.13) e (1.21) obtemos (1.48) e (1.49).

Seja $S$ o operador forma associado a $I I$, isto é, $H(X, Y)=\langle S X, Y\rangle$. Iremos ver como $S$ atua sobre o campo $\partial_{z}$. Tomemos $S \partial_{z}=a \partial_{z}+b \partial_{\bar{z}}$, onde $a, b$ são funções complexas diferenciáveis locais. Então, usando (1.47) e (1.48), temos

$$
Q=\left\langle S \partial_{z}, \partial_{z}\right\rangle=b \lambda \quad \text { e } \quad \lambda H=\left\langle S \partial_{z}, \partial_{\bar{z}}\right\rangle=a \lambda
$$

Então obtemos (1.50). Analogamente temos também $S \partial_{\bar{z}}=\frac{\bar{Q}}{\lambda} \partial_{z}+H \partial_{\bar{z}}$.

Dê (1.34)-(1.36) e usando que $p=0$, obtemos (1.51) e (1.52).

Agora da definição (1.3.1) e $\left[\partial_{z}, \partial_{\bar{z}}\right]=0$ temos

$$
T_{S}\left(\partial_{z}, \partial_{\bar{z}}\right)=\nabla_{\partial_{z}}\left(\frac{\bar{Q}}{\lambda} \partial_{z}+H \partial_{\bar{z}}\right)-\nabla_{\partial_{\bar{z}}}\left(H \partial_{z}+\frac{Q}{\lambda} \partial_{\bar{z}}\right) .
$$


De (1.51), (1.52) e (1.31)-(1.33) tem-se

$$
\begin{aligned}
T_{S}\left(\partial_{z}, \partial_{\bar{z}}\right) & =\left[\left(\frac{\bar{Q}}{\lambda}\right)_{z}+\frac{\bar{Q}}{\lambda^{2}} \lambda_{z}-H_{\bar{z}}\right] \partial_{z}-\left[\left(\frac{Q}{\lambda}\right)_{z}+\frac{Q}{\lambda^{2}} \lambda_{z}-H_{z}\right] \partial_{\bar{z}} \\
& =-H_{\bar{z}} \partial_{z}+H_{z} \partial_{\bar{z}}+\frac{\bar{Q}_{z}}{\lambda} \partial_{z}-\frac{Q_{\bar{z}}}{\lambda} \partial_{\bar{z}} \\
& =T_{H}\left(\partial_{z}, \partial_{\bar{z}}\right)+\frac{1}{\lambda}\left(\bar{Q}_{z} \partial_{z}-Q_{\bar{z}} \partial_{\bar{z}}\right) .
\end{aligned}
$$

Logo, obtemos (1.53). Na última igualdade usamos

$$
T_{H}\left(\partial_{z}, \partial_{\bar{z}}\right)=-H_{\bar{z}} \partial_{z}+H_{z} \partial_{\bar{z}}
$$

A equação (1.54) é consequência direta de (1.53) produto escalar com $\partial_{z}$. Finalmente, a curvatura Gaussiana é imediata de (1.46), a partir dos símbolos de Christoffel e $p=0$

Quando $I I$ é uma métrica Riemanniana, ou seja, quando a curvatura extrínseca do par $(I, I I)$ satisfaz $K>0$, o par $(I I, I)$ é um par fundamental. Assim, podemos também considerar um parâmetro conforme $z$ para $I I$. Neste caso temos $Q=0 \mathrm{em}$ (1.13). Para este parâmetro temos a seguinte proposição;

Proposição 1.6.3. Seja $(I, I I) \in \mathcal{P}(M)$, onde $I I$ é Riemanniana. Sejam $S^{I} \in \mathcal{S}(M, I I)$ o endomorfismo autoadjunto associado a $I$ com respeito a $I I$ e $S^{I I} \in \mathcal{S}(M, I)$ o endomorfismo autoadjunto associado a II com respeito a I. Seja z um parâmetro conforme para II, isto é,

$$
\begin{aligned}
I & =p d z^{2}+2 \lambda|d z|^{2}+\bar{p} d \bar{z}^{2} \\
I I & =2 \rho|d z|^{2}
\end{aligned}
$$

e denotemos $D=|p|^{2}-\lambda^{2}$. Então se verificam as seguintes equações:

$$
\begin{aligned}
\lambda K & =\rho H \\
S^{I} \partial_{z} & =\frac{H}{K} \partial_{z}+\frac{p}{\rho} \partial_{\bar{z}} \\
S^{I I} \partial_{z} & =K\left(\frac{H}{K} \partial_{z}-\frac{p}{\rho} \partial_{\bar{z}}\right) \\
\Gamma_{11}^{1}+\Gamma_{12}^{2} & =\frac{D_{z}}{2 D} \\
T_{S^{I I}}\left(\partial_{z}, \partial_{\bar{z}}\right) & =\frac{K_{z}}{2} S^{I} \partial_{\bar{z}}-\frac{K_{\bar{z}}}{2} S^{I} \partial_{z}-\frac{\left(K_{\bar{p}}\right)_{z}}{\rho} \partial_{z}+\frac{\left(K_{p}\right)_{\bar{z}}}{\rho} \partial_{\bar{z}} \\
\left\langle T_{S^{I I}}\left(\partial_{z}, \partial_{\bar{z}}\right), \partial_{z}\right\rangle & =2 \rho\left(\frac{K_{z}}{4 K}+\Gamma_{12}^{2}\right) .
\end{aligned}
$$

Demonstração. A equação (1.58) é uma consequência da definição de $H(I, I I)$ e $K(I, I I)$, isto é, (1.20) e (1.21) quando $Q \equiv 0$. Escrevendo $S^{I} \partial_{z}=a \partial_{z}+b \partial_{\bar{z}}$ para certas funções locais $a, b$ e 
usando (1.56) e (1.57), obtemos

$$
\begin{gathered}
p=I\left(\partial_{z}, \partial_{z}\right)=I I\left(S^{I} \partial_{z}, \partial_{z}\right)=b \rho \\
\lambda=I\left(\partial_{z}, \partial_{\bar{z}}\right)=I I\left(S^{I} \partial_{z}, \partial_{\bar{z}}\right)=a \rho
\end{gathered}
$$

Logo $b=\frac{p}{\rho}$ e $a=\frac{\lambda}{\rho}$. Então, de (1.58), obtemos a expressão (1.59).

Analogamente, se $S^{I I} \partial_{z}=a \partial_{z}+a \partial_{\bar{z}}$, então

$$
\begin{gathered}
0=I I\left(\partial_{z}, \partial_{z}\right)=I\left(S^{I I} \partial_{z}, \partial_{z}\right)=a p+b \lambda \\
\rho=I I\left(\partial_{z}, \partial_{\bar{z}}\right)=I\left(S^{I I} \partial_{z}, \partial_{\bar{z}}\right)=a \lambda+b \bar{\rho}
\end{gathered}
$$

Multiplicando (1.64) por $\bar{p},(1.65)$ por $\lambda$ e lembrando que $Q \equiv 0$, temos:

$$
a=\frac{\lambda \rho}{\lambda^{2}-|p|^{2}}=H \quad \text { e } \quad b=-\frac{p K}{\rho}
$$

Daí,

$$
S^{I I} \partial_{z}=H \partial_{z}-\frac{p K}{\rho} \partial_{\bar{z}}=K\left(\frac{H}{K} \partial_{z}-\frac{p}{\rho} \partial_{\bar{z}}\right)
$$

A equação (1.61) é nada mais que a soma de (1.34) e (1.39).

Seja $D=|p|^{2}-\lambda^{2}$, podemos reescrever a curvatura extrínseca como $-\frac{\rho^{2}}{D}$ donde derivando em relação a $z$ temos:

$$
\rho \frac{K_{z}}{2 K}=\rho_{z}-\rho \frac{D_{z}}{2 D} .
$$

A equação (1.63) segue de

$$
\begin{aligned}
\left\langle T_{S^{I I}}\left(\partial_{z}, \partial_{\bar{z}}\right), \partial_{z}\right\rangle & =\rho_{z}+\rho\left(\Gamma_{12}^{2}-\Gamma_{11}^{1}\right) \\
& =\rho_{z}+\rho\left(\Gamma_{12}^{2}-\frac{D_{z}}{2 D}+\Gamma_{12}^{2}\right) \\
& =\rho_{z}+2 \rho \Gamma_{12}^{2}-\rho \frac{D_{z}}{2 D} \\
& =\rho_{z}+2 \rho \Gamma_{12}^{2}+\rho \frac{K_{z}}{2 K}-\rho_{z} \\
& =2 \rho\left(\Gamma_{12}^{2}+\frac{K_{z}}{4 K}\right)
\end{aligned}
$$

onde usamos (1.61) e (1.66). 
Denotando $\alpha:=\left\langle T_{S^{I I}}\left(\partial_{z}, \partial_{\bar{z}}\right), \partial_{\bar{z}}\right\rangle$, podemos concluir de (1.44),(1.45) e (1.31)-(1.33) que $-\bar{\alpha}=$ $\left\langle T_{S^{I I}}\left(\partial_{z}, \partial_{\bar{z}}\right), \partial_{\bar{z}}\right\rangle$, e escrevendo $T_{S}\left(\partial_{z}, \partial_{\bar{z}}\right)=a \partial_{z}+b \partial_{\bar{z}}$, temos

$$
\alpha=a p+b \lambda \quad e \quad-\bar{\alpha}=a \lambda+b \bar{p} .
$$

Logo

$$
a=\frac{\bar{p} \alpha+\lambda \bar{\alpha}}{D} \quad e \quad b=-\frac{p \bar{\alpha}+\lambda \alpha}{D} .
$$

Então,

$$
\begin{aligned}
a & =\frac{1}{D}\left[\bar{p}\left(2 \rho\left(\frac{K_{z}}{4 K}+\Gamma_{12}^{2}\right)\right)+\lambda\left(2 \rho\left(\frac{K_{\bar{z}}}{4 K}+\Gamma_{12}^{1}\right)\right)\right] \\
& =\frac{2 \rho}{D}\left[\bar{p} \frac{K_{z}}{4 K}+\frac{\lambda K_{\bar{z}}}{4 K}+\bar{p} \Gamma_{12}^{2}+\lambda \Gamma_{12}^{1}\right] \\
& =-\frac{1}{2 \rho}\left[\bar{p} K_{z}+\lambda K_{\bar{z}}+2 \bar{p}_{z} K\right] \\
& =-\frac{(K \bar{p})_{z}}{\rho}-\frac{1}{2}\left(\frac{H K_{\bar{z}}}{K}-\frac{\bar{p} K_{z}}{\rho}\right)
\end{aligned}
$$

e

$$
b=-a=\frac{(K p)_{\bar{z}}}{\rho}+\frac{1}{2}\left(\frac{H K_{z}}{K}-\frac{p K_{\bar{z}}}{\rho}\right) .
$$

Finalmente, obtemos

$$
\begin{aligned}
T_{S^{I I}}\left(\partial_{z}, \partial_{\bar{z}}\right)= & -\frac{1}{\rho}\left((K \bar{p})_{z} \partial_{z}-(K p)_{\bar{z}} \partial_{z}\right) \\
& -\frac{K_{\bar{z}}}{2}\left(\frac{H}{K} \partial_{z}+\frac{p}{\rho} \partial_{\bar{z}}\right)+\frac{K_{\bar{z}}}{2}\left(\frac{H}{K} \partial_{\bar{z}}+\frac{\bar{p}}{\rho} \partial_{z}\right) \\
= & -\frac{1}{\rho}\left((K \bar{p})_{z} \partial_{z}-(K p)_{\bar{z}} \partial_{\bar{z}}\right)+\frac{1}{2}\left(K_{z} S^{I} \partial_{z}-K_{\bar{z}} S^{I} \partial_{z}\right)
\end{aligned}
$$

\subsubsection{A diferencial de Hopf}

Relembraremos o conceito de pontos umbílicos, no qual irá desempenhar um papel importante nos capítulos seguintes.

Definição 1.6.6. Dizemos que um par fundamental $(I, I I)$ é umbílico em $p \in M$ se II é proporcional a I ou, equivalentemente:

- se ambas as curvaturas principais coincidem em p, ou

- se o operador forma $S$ satisfaz $S=\alpha$ Id em $p$, tal que $\alpha \in \mathbb{R}$ ou

- se $H(I, I I)^{2}=K(I, I I)$ em $p$. 
Definição 1.6.7. Sejam z um parâmetro conforme local e $(I, I I)$ um par fundamental em $M$ dada por (1.47) e (1.48). Dizemos que a forma quadrática $Q d z^{2}$ de II é a diferencial de Hopf do par fundamental $(I, I I)$.

A diferencial de Hopf nos permite calcular os pontos umbílicos do par fundamental $(I, I I)$ :

Proposição 1.6.4. Sejam $(I, I I)$ um par fundamental em $M$ e z um parâmetro conforme local em $M$. Então, $p \in M$ é um ponto umbílico do par $(I, I I)$ se, e somente, se $Q(p)=0$.

Demonstração. Decorre de (1.47) e (1.48) que existe $\alpha \in \mathbb{R}$ tal que $I I=\alpha I$ em $p$ se, e somente se $Q(p)=0$.

Lema 1.6.1. O par $(I, I I)$ é de Codazzi se, e somente se, $H_{z}=\frac{Q_{\bar{z}}}{\lambda}$.

Demonstração. Seja $(I, I I)$ par de Codazzi. Então

$$
\nabla_{X} S Y-\nabla_{Y} S X-S[X, Y]=0 .
$$

A equação acima é equivalente a $\nabla_{\partial_{z}} S\left(\partial_{\bar{z}}\right)=\nabla_{\partial_{\bar{z}}} S\left(\partial_{z}\right)$, em que $\left\{\partial_{\bar{z}}, \partial_{z}\right\}$ é o referencial canônico de $T^{\mathbb{C}} M$ associado ao parâmetro conforme local $z$. Lembramos que $\left[\partial_{\bar{z}}, \partial_{z}\right]=0$. De (1.50) segue que

$$
\begin{aligned}
\nabla_{\partial_{z}} S \partial_{\bar{z}} & =\nabla_{\partial_{z}}\left(\frac{\bar{Q}}{\lambda} \partial_{z}\right)+\nabla_{\partial_{z}}\left(H \partial_{\bar{z}}\right) \\
& =\frac{\bar{Q}}{\lambda} \nabla_{\partial_{z}} \partial_{z}+\left(\frac{\bar{Q}}{\lambda}\right)_{z} \partial_{z}+H \nabla_{\partial_{z}} \partial_{\bar{z}}+H_{z} \partial_{\bar{z}}
\end{aligned}
$$

De (1.51) e (1.52) obtemos

$$
\begin{aligned}
\nabla_{\partial_{z}} S \partial_{\bar{z}} & =\frac{\bar{Q} \lambda_{z}}{\lambda^{2}} \partial_{z}+\frac{\bar{Q}_{z} \lambda-\bar{Q} \lambda_{z}}{\lambda^{2}} \partial_{z}+H_{z} \partial_{\bar{z}} \\
& =\frac{\bar{Q}_{z}}{\lambda} \partial_{z}+H_{z} \partial_{\bar{z}}
\end{aligned}
$$

De forma análoga, encontramos $\nabla_{\partial_{\bar{z}}} S \partial_{z}=H_{\bar{z}} \partial_{z}+\frac{Q_{\bar{z}}}{\lambda} \partial_{\bar{z}}$, portanto

$$
\frac{\bar{Q}_{z}}{\lambda} \partial_{z}+H_{z} \partial_{\bar{z}}=H_{\bar{z}} \partial_{z}+\frac{Q_{\bar{z}}}{\lambda} \partial_{\bar{z}}
$$




\section{Superfícies Completas com Curvatura Extrínseca Não-positiva em $\mathbb{H}^{3}$ e $\mathbb{S}^{3}$}

Neste capítulo, baseado nos estudos de José Galvez, Antônio Martínez e José Teruel, em [21] e [20], utilizaremos a técnica de pares de Codazzi para demonstrar um resultado abstrato para pares de Codazzi em superfícies de curvatura extrínseca não-positiva e o aplicaremos para estudar uma solução parcial para conjectura de Milnor e resultados do tipo Efimov nas formas espaciais não-Euclidianas $\mathbb{H}^{3}$ e $\mathbb{S}^{3}$.

\subsection{Introdução}

Muitos resultados clássicos da teoria de superfícies em $\mathbb{R}^{3}$ são consequências da equação de Codazzi, que continua sendo válidas em $\mathbb{H}^{3}$ e $\mathbb{S}^{3}$. Portanto, não é surpreendente que resultados da teoria de superfícies imersas em $\mathbb{R}^{3}$ podem ser aplicados no contexto abstrato dos pares de Codazzi. É por isso que pares de Codazzi aparecem naturalmente na teoria de subvariedades, e que a primeira e segunda formas fundamentais de qualquer superfície imersa em uma forma espacial tridimensional é um par de Codazzi. Está por sua vez, será a ferramenta principal para obter resultados tipo Milnor e do tipo Efimov em superfícies completas imersas em espaços de curvatura constante não-nula.

Neste contexto, apresentaremos um resultado abstrato para pares de Codazzi, dado por uma generalização do teorema de Smyth-Xavier [20], que também será apresentado neste capítulo. Considerando imersões para os espaços tridimensionais $\mathbb{H}^{3}$ e $\mathbb{S}^{3}$, veremos que não existem imersões completas em $\mathbb{H}^{3}$ (resp. $\mathbb{S}^{3}$ ) com curvatura $K \leq-1$ (resp. $K \leq c<0$ ), sempre e quando uma de suas curvaturas principais é separada de zero. Além disso, nas condições referidas anteriormente sobre as curvaturas, a área dos fins de uma superfície imersa em uma forma espacial tridimensional é finita para a métrica induzida. 


\subsection{Definições e Resultados Básicos}

Consideramos $\psi$ uma $C^{2}$-imersão de uma superfície $S$ no espaço Euclidiano tridimensional. Podemos supor $S$ orientável, caso contrário, recorremos ao recobrimento orientável das parametrizações.

Seja $N: S \longrightarrow \mathbb{S}^{2}$ a aplicação normal de Gauss, tal que $A=-d N$ é o endomorfismo de Weingarten da imersão e seja $N(S)$ a imagem esférica da superfície. Denotaremos por $k_{1}$ e $k_{2}$ as respectivas curvaturas principais de $\psi$, isto é, os autovalores de $A$.

Denotaremos por $K^{-}:=\min [K, 0]$ e $K^{+}:=\max [K, 0]$ as partes negativa e positiva da curvatura de $S$, respectivamente, e também, se existir, chamaremos curvatura total da superfície o valor

$$
\zeta:=\int_{S} K^{+} d A-\int_{S} K^{-} d A \equiv \int_{S}|K| d A
$$

Definição 2.2.1. Consideramos $S$ uma superfície topológica. Dizemos que $S$ tem topologia finita se é homeomorfa a uma superfície compacta menos um conjunto finito de pontos.

Definição 2.2.2. Uma superfície Riemanniana $S$ é dita do tipo parabólico se as únicas funções sub-harmônicas negativas sobre $S$ são as constantes.

Usando uma abordagem diferente, veremos nas próximas seções, que é possível obter uma versão do teorema e da conjectura a seguir, em formas espaciais não-Euclidianas, na qual dependem apenas da equação de Codazzi.

Teorema 2.2.1 (Efimov [25]). Nenhuma superfície $S$ pode ser imersa no espaço Euclidiano $\mathbb{R}^{3}$, tal que na métrica induzida $S$ seja completa e tenha curvatura Gaussiana $K \leq$ const $<0$.

Conjectura 2.2.1 (Jonh Milnor [24]). Suponha S superfície completa, sem pontos umbílicos, $C^{2}$-imersa em $\mathbb{R}^{3}$ tal que

$$
k_{1}^{2}+k_{2}^{2} \geq c>0
$$

onde $k_{i}, i=1,2$ são as curvaturas principais da imersão e c é uma constante qualquer. Então, ou a curvatura de Gauss $K$ muda de sinal ou $K \equiv 0$.

Definição 2.2.3. Dizemos que um mergulho é próprio se dada uma aplicação $f: X \longrightarrow Y$ tal que $f(\partial X)=f(x) \cap \partial Y$ e $f(X)$ é transversal a $\partial Y$ em qualquer ponto de $f(\partial X)$. A primeira condição é equivalente a ter $f(\partial X) \subseteq Y \backslash \partial Y$; a segunda condição, a grosso modo, diz que $f(X)$ não é tangente à fronteira, isto é, os espaços se cruzam.

Seguem abaixo alguns resultados dos trabalhos de Alfred Hüber [22] que nos serão de grande utilidade ao longo do capítulo. 
Teorema 2.2.2 (Hüber, 1957). Seja S uma superfície Riemanniana conexa, completa e orientada. Se

$$
\int_{S}\left|K^{-}\right|<+\infty
$$

então S tem topologia finita e

$$
\int_{S} K^{+} d A \leq 2 \pi \chi(S)
$$

onde $\chi$ é característica de Euler de $S$. Além disso $S$ é do tipo parabólico.

Como consequência do teorema acima, podemos obter o seguinte teorema de classificação:

Teorema 2.2.3 (Hüber). Seja S uma superfície Riemanniana conexa, completa, não-compacta e de curvatura de Gauss não-negativa. Então uma das seguintes possibilidades ocorre:

1) S é topologicamente um plano;

2) S é plana e, portanto, é um quociente do plano Euclidiano $\mathbb{R}^{2}$

Observação 2.2.1 (Ver [10]). A importância de S ser do tipo topológico finito é utilizada no resultado de Cohn-Vossen para superfícies completas não-compactas. Sabemos que para uma superfície $(S, g)$ compacta, orientada e conexa, o clássico teorema de Gauss-Bonnet* ${ }^{*}$, afirma que

$$
\int_{S} K d A=2 \pi \mathcal{X}(S)
$$

Porém, no caso de superficies não-compactas o tratamento é delicado. O primeiro a considerar este problema foi S. Cohn-Vossen em 1935. Cohn-Vossen supôs primeiramente S completa. Posteriormente, assumiu que o valor da característica de Euler da superfície completa $\mathcal{X}(S)$ e $\int_{S} K d A$ não eram necessariamente números finitos. Admitiu ainda que $S$ é do tipo topológico finito, isto é, $S$ é homeomorfa a uma superfície fechada $\bar{S}$ com um número finito $k$ de pontos removidos (chamados de fins), $k \geq 1$. Então, em particular $\mathcal{X}(S)=\mathcal{X}(\bar{S})-k=\mathcal{X}(\bar{S})-1 \leq 1$. Por último, considerou que a curvatura de Gauss K era absolutamente integrável em $S$, ou seja, $\int_{S}|K| d A<\infty$. Então propôs o seguinte resultado:

Teorema 2.2.4. Se $(S, g)$ é uma superfície completa do tipo topológico finito e $\int_{S}|K| d A<\infty$, então

$$
\int_{S}|K| d A \leq 2 \pi \mathcal{X}(S)
$$

Em particular, temos

$$
\int_{S}|K| d A \leq 2 \pi
$$

se $S$ é não-compacta.

Teorema 2.2.5 (Sacksteder [31]). Seja $\psi: M^{n} \longrightarrow \mathbb{R}^{n+1}, n>1$, uma imersão isométrica de uma variedade Riemanniana $M, n$-dimensional, de classe $C^{\infty}$, completa e orientável no espaço $\mathbb{R}^{n+1}$

${ }^{*}$ Ver [8], cap. 4, seção 4.5 
cujas curvaturas seccionais são não-negativas e, pelo menos em um ponto, são todas positivas. Então $\psi(M)$ é uma subvariedade convexa. Em particular, $\psi$ mergulha $M$ topologicamente como um subconjunto fechado de $\mathbb{R}^{n+1}$. Além disso, $M$ ou é homeomorfa à esfera $\mathbb{S}^{n}$ ou ao $\mathbb{R}^{n+1}$

Observação 2.2.2. Dizemos que um subconjunto $N$ de uma variedade $M$ é convexo se dados $p$ e q pertencentes a $N$, existe uma geodésica minimal de $M$ ligando $p$ a q, a qual esta inteiramente contida em $N$. Se, além disso, o interior de $N$ é não vazio, definimos $N$ como um corpo convexo. Uma subvariedade $N$ de uma variedade Riemanniana $M$ é uma subvariedade convexa se $N$ é o bordo de um corpo convexo em $M$.

Definição 2.2.4 (Variedade Hadamard). Uma variedade de Hadamard é uma variedade Riemanniana $M$ conexa, completa, simplesmente conexa e com curvatura seccional não-positiva.

Teorema 2.2.6 (Hadamard [9]). Seja $M$ uma variedade Riemanniana completa, simplesmente conexa, com curvatura seccional $K(p, M) \leq 0, \forall p \in M$ e todo $M \subset T_{p}(M)$. Então $M$ é difeomorfo a $\mathbb{R}^{n}, n=\operatorname{dim} M$; mais precisamente, $\exp _{p}: T_{p} M \longrightarrow M$ é um difeomorfismo, em que $\exp _{p}$ é a aplicação exponencial.

Corolário 2.2.1. Qualquer superfície completa, simplesmente conexa $C^{2}$ - imersa com curvatura $K \leq 0$ tem área infinita.

Demonstração. A prova consiste na construção de coordenadas polares $(\rho, \theta)$ em $S$, e mostrando que a primeira forma fundamental $I$ é dada por $d \rho^{2}+\bar{G} d \theta^{2}$ onde $\bar{G}(\rho, \theta)$ é uma função contínua satisfazendo $\sqrt{\bar{G}} \geq \rho$ para todo $\rho>0$ e $\theta$. Da existência de tais coordenadas polares, segue então

$$
A(S)=\int_{0}^{2 \pi} \int_{0}^{\infty} \sqrt{\bar{G}} d \rho d \theta \geq \int_{0}^{2 \pi} \int_{0}^{\infty} \rho d \rho d \theta=\infty
$$

Para isso, fixamos $p$ em $S$ e seja $v=\gamma^{\prime}(0) \in T_{p} S$. Sabemos que existe uma única geodésica tal que $\gamma(0)=p$ e $v=\gamma^{\prime}(0)$. Além disso, como exp $: T_{p} S \longrightarrow S$ é um difeomorfismo local, ela induz um produto interno em $T_{p} S$. Denotamos por $T$ a superfície geométrica em $T_{p} S$ com este produto.

Se introduzirmos coordenadas cartesianas $(x, y)$ no espaço tangente $T$, podemos escrever este produto interno como $\bar{I}=E d x^{2}+2 f d x d y+G d y^{2}$ sobre $T$, onde $E, F$ e $G$ são funções contínuas.

Agora introduzindo sistema de coordenadas polares em $T$, em termos do qual $x=r \cos (\theta)$, $y=r \sin (\theta)$, podemos reescrever $\bar{I}$ como $d \rho^{2}+\bar{G} d \theta^{2}$, tal que $\bar{E}(\rho, \theta)=1, \bar{F}(\rho, \theta)=0, \lim _{\rho \rightarrow 0} \bar{G}=0$ e $\lim _{\rho \rightarrow 0}(\sqrt{\bar{G}})_{\rho}=1$. Segue daí que a curvatura de Gauss pode ser escrita como:

$$
K=-\frac{1}{2 \sqrt{G}}\left(\frac{\bar{G}_{\rho}}{\sqrt{G}}\right)_{\rho}=-\frac{1}{\sqrt{G}}\left(\frac{\bar{G}_{\rho}}{2 \sqrt{G}}\right)_{\rho} .
$$

Observe que

$$
(\sqrt{\bar{G}})_{\rho}=\frac{\bar{G}_{\rho}}{2 \sqrt{\bar{G}}}
$$


Logo

$$
K=-\frac{(\sqrt{\bar{G}})_{\rho \rho}}{\sqrt{\bar{G}}}
$$

Se $K \leq 0$ sobre $S$, então a equação $(2.1)$ implica que $(\sqrt{\bar{G}})_{\rho \rho} \geq 0$. Daí $(\sqrt{\bar{G}})_{\rho}$ é uma função não decrescente. $\operatorname{Logo}(\sqrt{\bar{G}})_{\rho} \geq \lim _{\rho \rightarrow 0}(\sqrt{\bar{G}})_{\rho}=1$ e, então, $\sqrt{\bar{G}} \geq \rho, \forall \rho$ e $\theta$.

Como $\bar{G} \geq 0$, temos que a métrica $\bar{I}=d \rho^{2}+\bar{G} d \theta^{2}$ é maior ou igual a métrica Euclidiana $I=d \rho^{2}+\rho d \theta^{2}$ em $T$. Portanto exp é uma aplicação recobrimento. Assim, desde que $S$ seja simplesmente conexa, a função recobrimento exp é $C^{1}$-difeomorfismo. Seja a área de $T$ infinita na métrica $\bar{I}, S$ deve ter área infinita na métrica $I$.

Observação 2.2.3. Note que o corolário anterior poderia ser facilmente provado usando o fato de $S$ ser uma variedade de Hadamard.

\subsection{Uma Solução Parcial da Conjectura de Milnor}

O teorema que iremos enunciar a seguir é uma solução parcial da conjectura de Milnor (ver [20]), em $\mathbb{R}^{3}$ no caso de $K \leq 0$ obtida por Smyth-Xavier.

Teorema 2.3.1 (Smyth-Xavier). Seja $\psi: S \longrightarrow \mathbb{R}^{3}$ uma superfície completa imersa isometricamente, de curvatura não-positiva. Se uma das suas curvaturas principais $k_{i}, i=1,2$ satisfaz $k_{i}^{2} \geq$ const $>0$, então $\psi(S)$ é um cilindro generalizado em $\mathbb{R}^{3}$.

Demonstração. Sejam $k_{1}$ e $k_{2}$ as curvaturas principais de $\psi(S)$. A menos de uma mudança de orientação, podemos assumir que

$$
k_{2} \geq \epsilon>\frac{\epsilon}{2}>0>k_{1},
$$

para alguma constante positiva $\epsilon$. Considere $\psi_{\epsilon}$ uma aplicação paralela de $\psi$ para uma distância $2 / \epsilon$, tal que

$$
\psi_{\epsilon}=\psi+\frac{2}{\epsilon} N
$$

onde $N: S \longrightarrow \mathbb{S}^{2}$ é a aplicação de Gauss de $\psi$. Note que $\psi_{\epsilon}$ é uma imersão, pois $N_{\epsilon} \neq 0$, na qual induz uma métrica $\Lambda_{\epsilon}=<d \psi_{\epsilon}, d \psi_{\epsilon}>$, dada por $\Lambda_{\epsilon}=I-\frac{4}{\epsilon} I I+\frac{4}{\epsilon^{2}}$. Segue de

$$
\begin{aligned}
I & =E d u^{2}+G d v^{2} \\
I I & =k_{1} E d u^{2}+k_{2} G d v^{2} \\
I I I & =k_{1}^{2} E d u^{2}+K_{2}^{2} G d v^{2}
\end{aligned}
$$

que

$$
\Lambda_{\epsilon}=\left(1-\frac{2}{\epsilon} k_{1}\right)^{2} E d u^{2}+\left(1-\frac{2}{\epsilon} k_{2}\right)^{2} G d v^{2}
$$




$$
d A_{\Lambda_{\epsilon}}=\left|\left(1-\frac{2}{\epsilon} k_{1}\right)\left(1-\frac{2}{\epsilon} k_{2}\right)\right|=-\frac{1}{\epsilon^{2}}\left(\epsilon-2 k_{1}\right)\left(\epsilon-2 k_{2}\right) d A
$$

onde $I, I I, I I I$ denotam a primeira, segunda e terceira formas fundamentais de $\psi$, respectivamente. Ademais $d A$ e $d A_{\epsilon}$ são os elementos área de $I$ e $\Lambda_{\epsilon}$, respectivamente.

Notemos que as curvaturas principais de $\psi_{\epsilon}, k_{1}^{\epsilon}$ e $k_{2}^{\epsilon}$, podem serem escritas como

$$
k_{1}^{\epsilon}=\frac{\epsilon}{\epsilon-2 k_{1}} \quad \text { e } \quad k_{2}^{\epsilon}=\frac{\epsilon}{\epsilon-2 k_{2}} .
$$

Então, a curvatura de Gauss, $K\left(\Lambda_{\epsilon}\right)$, de $\psi_{\epsilon}$ é dada por

$$
K\left(\Lambda_{\epsilon}\right)=\frac{K(I)}{\left(1-\frac{2}{\epsilon} k_{1}\right)\left(1-\frac{2}{\epsilon} k_{2}\right)}
$$

onde $K(I)$ é a curvatura de Gauss de $I$.

Percebamos também que

$$
K\left(\Lambda_{\epsilon}\right)=\frac{\epsilon^{2} K(I)}{\left(\epsilon-2 k_{1}\right)\left(\epsilon-2 k_{2}\right)} \geq 0,
$$

pois $K(I)$ é não positiva por hipótese.

Observe que de (2.2) temos:

$$
\begin{gathered}
k_{2} \geq \epsilon>\frac{\epsilon}{2}>0>k_{1} \Longrightarrow \frac{2 k_{2}}{\epsilon} \geq 2>1>0>\frac{2 k_{1}}{\epsilon} \\
\Longrightarrow \frac{-2 k_{2}}{\epsilon}<-2<-1<0 \leq \frac{-2 k_{1}}{\epsilon} \Longrightarrow \frac{-2 k_{2}}{\epsilon}+1<-1<0<1 \leq \frac{-2 k_{1}}{\epsilon}+1
\end{gathered}
$$

e assim, de (2.3) temos $I \leq \Lambda_{\epsilon}$.

Note ainda que de (2.4), $\psi_{\epsilon}$ é uma imersão completa em $\mathbb{R}^{3}$ de curvatura não negativa. Agora, pelo Teorema de Sacksteder, ou $\psi_{\epsilon}(S)$ é um cilindro generalizado ou sua curvatura não é identificamente nula e, assim, $\psi_{\epsilon}$ é um mergulho convexo satisfazendo um dos seguintes itens:

(a) $S$ é homeomorfo à esfera ou;

(b) $S$ é homeomorfo ao plano.

Veremos que os casos $(a)$ e $(b)$ não podem ocorrer. De fato, o caso $(a)$ não é possível porque qualquer superfície compacta em $\mathbb{R}^{3}$ deve ter pelo menos um ponto elíptico, o que contradiz nossa hipótese sobre $\psi$. 
Para o caso (b) observe que $\psi_{\epsilon}$ é uma imersão própria. Segue da convexidade global, que existe um cone $V_{\epsilon} \operatorname{com} Z$ eixo de rotação tal que $\psi_{\epsilon}(S)$ está contido no cone $V_{\epsilon}$. Mas, $\psi$ é obtida de $\psi_{\epsilon}$ como uma superfície paralela com distância $2 / \epsilon$. Então $\psi$ também é uma imersão própria e $\psi_{S}$ está contida em um cone $V$ obtido como uma superfície paralela de $\psi_{\epsilon}(S)$ com uma distância $2 / \epsilon$. Sob essas condições, assumimos que $\psi$ deve ter pelo menos um ponto elíptico, o que também seria uma contradição.

Para ver isso, podemos assumir que, a menos de uma translação vertical, o vértice de $V$ é a origem e como $\psi$ é ima imersão isométrica e está contida em $V$, a distância $\psi(S)$ da origem é um número real positivo $d_{0}>0$. Considere uma capa esférica $S_{-}^{2}\left(R, 2 d_{0}\right)$ passando pela origem, de altura $2 d_{0}$ e fronteira do círculo de raio $R$ obtido pela secção $V \cap\left\{Z=2 d_{0}\right\}$. Pela construção $\psi(S) \cap S_{-}^{2}\left(R, 2 d_{0}\right)=\emptyset$. Assim, fixando este círculo e escolhendo a capa esférica $S_{-}^{2}\left(R, 2 d_{0}-t\right)$ de altura $2 d_{0}-t, 0 \leq t \leq 2 d_{0}$ passando por $\left(0,0, t_{0}\right)$ com fronteira do círculo mencionado, conseguimos um $t_{0}, d_{0} \leq t_{0} \leq 2 d_{0}$, onde $S_{-}^{2}\left(R, 2 d_{0}-t_{0}\right)$ intersecta a superfície $\psi(S)$ em um primeiro ponto. É claro que o ponto de interseção é elíptico.

Observe que o teorema acima constitui um caso particular das hipóteses da Conjectura de Milnor. De fato, se $k_{i}^{2} \geq$ cte $>0$, então $k_{1}^{2}+k_{2}^{2} \geq$ cte $>0$. Além disso, a hipótese $k_{i}^{2}>0$ e a curvatura não-positiva, implica que os pontos não são umbílicos. O resultado do teorema é um caso particular do resultado da Conjectura, isto é, se temos um cilindro generalizado, então a curvatura é zero.

Uma consequência interessante deste teorema é, por exemplo, que os cilindros generalizados são as únicas superfícies completas com curvatura de Gauss não-positiva em $\mathbb{R}^{3}$ com curvatura média limitada a partir de zero. Escrito de outra modo, temos o seguinte resultado:

Corolário 2.3.1. Seja $\psi: S \longrightarrow \mathbb{R}^{3}$ uma superficie completa, imersa, com curvatura de Gauss não-positiva e curvatura média $H$ tal que $|H| \geq$ const $>0$. Então $\psi(S)$ é um cilindro generalizado em $\mathbb{R}^{3}$.

Demonstração. Seja $H=\frac{k_{1}+k_{2}}{2}$ a curvatura média. Elevando ao quadrado os termos da desigualdade $|H| \geq$ const $>0$ então uma das curvaturas principais $k_{i}, i=1,2$ satisfaz $k_{i} \geq \epsilon>0$, para qualquer constante positiva $\epsilon$. Portanto pelo Teorema 2.3.1 segue o resultado.

\subsection{Pares de Codazzi em Superfícies Completas com Curvatura Não-positiva}

Muitos resultados clássicos da teoria de superfícies de $\mathbb{R}^{3}$ são consequência da equação de Codazzi, que continua valendo em outros espaços tridimensionais de curvatura contante, como $\mathbb{H}^{3}$ e $\mathbb{S}^{3}$. Nesta seção veremos resultados tipo Milnor e Efimov sobre superfícies completas, imersas em formas espaciais não Euclidianas os quais dependem apenas da equação de Codazzi. 
Assim, apresentaremos um resultado geral sobre pares de Codazzi e estenderemos este resultado à superfícies em $\mathbb{H}^{3}$ e $\mathbb{S}^{3}$.

Definição 2.4.1. Dizemos que as curvaturas principais $k_{1}$ e $k_{2}$ de um par fundamental de $\Sigma$ são estritamente separadas se existem números reais $c_{1}$ e $c_{2}$ tais que

$$
k_{1} \leqslant c_{1}<c_{2} \leqslant k_{2} .
$$

Teorema 2.4.1. Seja $(I, I I)$ um par de Codazzi em $\Sigma$ com curvaturas principais estritamente separadas. Se $(\Sigma, I)$ é uma superfície completa com curvatura de Gauss $K(I) \leqslant 0$, então apenas um dos seguintes itens é verificado:

1) I é uma métrica plana e $\Sigma$ é homeomorfa ou a um plano, ou a um cilindro ou a um toro plano.

2) I não é plana, $\Sigma$ é homeomorfo a um plano e

$$
\int_{\Sigma}|K(I)| d A_{I} \leqslant 2 \pi
$$

Demonstração. Consideremos a terceira forma fundamental III associada com o par $(I, I I)$ e $(u, v)$ parâmetros locais duplamente ortogonais. Então:

$$
I=E d u^{2}+G d v^{2} \quad \text { e } \quad I I=e d u^{2}+g d v^{2}
$$

O conjunto $\left\{\partial_{u}, \partial_{v}\right\}$ constitui uma base ortogonal das direções principais do plano tangente da superfície em cada ponto. Assim,

$$
k_{1}=\frac{e}{E} \quad \text { e } \quad k_{2}=\frac{g}{G} .
$$

Segue daí que podemos reescrever $I I$ como

$$
I I=k_{1} E d u^{2}+k_{2} G d v^{2} .
$$

Portanto, a terceira forma fundamental do par pode ser expressa como

$$
I I I=k_{1}^{2} E d u^{2}+k_{2}^{2} G d v^{2} .
$$

Estes tipos de coordenadas existem em torno de um ponto não umbílico de $\Sigma$ ou no interior do conjunto de seus pontos umbílicos. Se a $\in \mathbb{R} \backslash\{0\}$ satisfaz $a k_{1} \neq 1, a k_{2} \neq 1$ em $\Sigma$, e considerando a forma quadrática $\Lambda_{a}$ definida como (1.5), então $\Lambda_{a}$ é a métrica Riemanniana de $\Sigma$. Segue da $I, I I$ e $I I I$ formas fundamentais

$$
\Lambda_{a}=\left(1-a k_{1}\right)^{2} E d u^{2}+\left(1-a k_{2}\right)^{2} G d v^{2} .
$$


Além disso, pela Proposição 1.5.1 temos que a curvatura é calculada como

$$
K\left(\Lambda_{a}\right)=\frac{K(I)}{\left(1-a k_{1}\right)\left(1-a k_{2}\right)},
$$

e como as curvaturas principais são estritamente separadas, da condição (2.5), podemos escolher $a$ em $\mathbb{R} \backslash\{0\}$ tal que

$$
k_{1} \leq c_{1}<\frac{1}{a}<c_{2} \leq k_{2} .
$$

Segue daí que $1-a k_{1} \leq 1-a c_{1}<0<1-a c_{2} \leq 1-a k_{2}$, e, se tomarmos $c_{0}:=$ $\min \left\{\left|1-a c_{i}\right|, i=1,2\right\}$, então $\left(1-a k_{i}\right)^{2} \geq c_{o}^{2}, i=1,2$. Agora de (2.7) temos $\Lambda_{a} \geq c_{o}^{2}\left(E d u^{2}+\right.$ $\left.G d v^{2}\right)=c_{0}^{2}(I)$, o que implica que $\Lambda_{a}$ é completa, pois $I$ é completa. Agora segue de (2.8) que $K\left(\Lambda_{a}\right) \geq 0$.

Logo $\left(\Sigma, \Lambda_{a}\right)$ é uma superfície Riemanniana completa de curvatura não-negativa. Vamos analisar os dois casos:

1) $K\left(\Lambda_{a}\right)$ é identicamente nula. Neste caso, temos de (2.8) que $K(I) \equiv 0$, logo $I$ é métrica plana. Então pelo Proposição 1.2.2, podemos encontrar uma isometria $\phi: \mathbb{R}^{2} \longrightarrow \bar{\Sigma}$, onde $\bar{\Sigma}$ é o recobrimento universal de $\Sigma$. Portanto $\Sigma$ é homeomorfo ao quociente $\mathbb{R}^{2} / \Gamma$, onde $\Gamma$ é o grupo discreto de isometrias que atua propriamente sobre $\mathbb{R}^{2}$, e os únicos casos possíveis que são orientados são um plano, um cilindro ou um toro plano.

2) $\mathrm{K}\left(\Lambda_{a}\right)>0$. Neste caso, consideramos sobre $\Sigma$ a métrica Riemanniana conforme induzida por $\Lambda_{a}$. Usando o Teorema de Hüber sobre $\left(\Sigma, \Lambda_{a}\right)$, podemos afirmar que $\Sigma$ é conformemente um plano ou uma esfera. Mas, se $\Sigma$ é uma esfera, pelo teorema de Gauss-Bonnet ${ }^{\dagger}$, usando (2.7), (2.8) e o fato que $d A_{\Lambda_{a}}=\left|\left(1-a k_{1}\right)\left(1-a k_{2}\right)\right| d A_{I}$ temos

$$
4 \pi=\int_{\Sigma} K\left(\Lambda_{a}\right) d A_{\Lambda_{a}}=-\int_{\Sigma} K(I) d A_{I}=-4 \pi
$$

o que é uma contradição.

Seja $\Sigma$ não-compacta. Então deve ser homeomorfa a um plano e pelo Teorema de Hüber (2.2.2), usando (2.7) e (2.8) temos a seguinte inequação

$$
\int_{\Sigma}|K(I)| d A_{I}=\int_{\Sigma} K\left(\Lambda_{a}\right) d A_{\Lambda_{a}} \leq 2 \pi
$$

o que conclui a demostração.

Note que no contexto das $C^{2}$-imersões isométricas no espaço Euclidiano tridimensional, o par constituído pelas primeira e a segunda formas fundamentais da imersão é de Codazzi. Neste contexto, as condições do Teorema 2.4.1 são equivalentes àquelas do Teorema de Smyth-Xavier,

${ }^{\dagger}$ Ver [8], cap. 4, seção 4.5 
dado que o fato de ter curvaturas principais estritamente separadas e que uma delas limitada inferiormente por uma constante positiva é o mesmo quando $K \leq 0$.

Observe também que, se a superfície satisfaz às condições do teorema fora de um conjunto compacto, podemos aplicar o Teorema de Hüber 2.2.2 para obter que $(\Sigma, I)$ tem curvatura total finita. Vemos isso no seguinte resultado:

Teorema 2.4.2. Sejam $\Sigma$ uma superfície e $C \subset \Sigma$ um subconjunto compacto. Assuma que $(I, I I)$ é um par de Codazzi sobre $\Sigma \backslash C$, cujas curvaturas principais estão estritamente separadas. Se I é uma métrica completa com curvatura de Gauss não-positiva sobre $\Sigma \backslash C$, então $(\Sigma \backslash C, I)$ tem curvatura total finita. Em particular, $\Sigma$ é do tipo parabólico e tem topologia finita.

Demonstração. Similarmente ao teorema anterior, podemos chegar que $\left(\Sigma \backslash C, \Lambda_{a}\right)$ é completa e que $K\left(\Lambda_{a}\right) \geq 0$. Por $(2.7)$ e (2.8) temos o seguinte:

$$
\int_{\Sigma \backslash C}|K(I)| d A_{I}=\int_{\Sigma \backslash C} K\left(\Lambda_{a}\right) d A_{\Lambda_{a}}<\infty
$$

Portanto $\Sigma \backslash C$ tem curvatura total finita e, segue do Teorema de Hüber (2.2.2) que a superfície é parabólico e tem topologia finita.

\subsubsection{Superfícies Completas com $K \leq 0$ em $\mathbb{H}^{3}$ e $\mathbb{S}^{3}$}

Nesta seção aplicaremos os teoremas anteriores para obter resultados tipos Efimov e Milnor no espaço hiperbólico, $\mathbb{H}^{3}$ e na esfera, $\mathbb{S}^{3}$.

Como primeira consequência do Teorema 2.4.1 temos,

Corolário 2.4.1. Seja $\psi: \Sigma \longrightarrow \mathbb{H}^{3}$ uma imersão com curvatura de Gauss $K \leq-1$ e assumamos que uma das curvaturas principais $k_{i}$ satisfaz

$$
k_{i}^{2} \geq \epsilon^{2}>0, i=1,2
$$

para alguma constante $\epsilon>0$. Então $\psi$ não é uma imersão completa.

Demonstração. Pela fórmula de Gauss da imersão $\psi$ temos

$$
k_{1} k_{2}=K+1 \leq 0 .
$$

Logo, a menos de uma orientação, podemos assumir que $k_{2} \geq \epsilon>0$ e daí as curvaturas principais da imersão satisfazem $k_{2} \geq \epsilon>0 \geq k_{1}$. Sabemos que o par $(I, I I)$ formado pelas primeira e segunda formas fundamentais de $\psi$ é um par de Codazzi e as curvatura principais são estritamente separadas. 
Se assumirmos que $I$ é completa, então aplicando o Teorema 2.4.1, deduzimos que $\Sigma$ é homeomorfo a um plano e

$$
\int_{\Sigma} \mid K(I) d A_{I} \leqslant 2 \pi
$$

Por hipótese, temos $K \leq-1$. Logo, podemos estimar a da área de $\Sigma$ da seguinte forma

$$
\int_{\Sigma}|K| d A \geq A(\Sigma)
$$

Portanto de (2.10) e (2.11) temos que $2 \pi \geq A(\Sigma)$, o que é absurdo, pois sabemos, pelo Corolário 2.2.1, que qualquer superfície de Hadamard tem área infinita.

Impondo a separação estrita das curvaturas principais na imersão e argumentando de forma similar ao corolário anterior, podemos provar o seguinte resultado tipo Efimov sobre $\mathbb{H}^{3}$ como consequência direta do Teorema 2.4.1.

Corolário 2.4.2. Não existem superfícies completas imersas em $\mathbb{H}^{3}$ com curvatura de Gauss $K \leq-\epsilon<0$, para alguma constante $\epsilon>0$, e com curvaturas principais estritamente separadas.

Demonstração. Se assumirmos que a métrica induzida pela imersão $I$ é completa, pelo Teorema 2.4.1, temos que $\Sigma$ é homeomorfa ao plano e tem curvatura total finita. Segue da hipótese que $|K| \geq|\epsilon|>0$. Logo, pelos mesmos argumentos do corolário anterior, temos que $\frac{2 \pi}{|\epsilon|} \geq A(\Sigma)$, o que é absurdo, afinal, pelo Corolário 2.2.1, sabemos que qualquer superfície de Hadamard tem área infinita.

É possível obtermos um resultado análogo ao Corolário 2.4.1 para a esfera $\mathbb{S}^{3}$.

Corolário 2.4.3. Seja $\psi: \Sigma \longrightarrow \mathbb{S}^{3}$ uma imersão com curvatura de Gauss $K \leq$ const $<0$ e umas das curvaturas principais $k_{i}$ satisfaz

$$
k_{i}^{2} \geq \epsilon^{2}>0, \quad i=1,2,
$$

para alguma constante $\epsilon>0$. Então $\psi$ não é uma imersão completa.

Demonstração. Novamente, pela fórmula de Gauss da imersão $\psi$ e denotando por $c$ uma constante qualquer, temos

$$
k_{1} k_{2}=K-1 \leq-1
$$

Logo, a menos de uma orientação, podemos assumir que $k_{2} \geq \epsilon>0$ e daí as curvaturas principais da imersão satisfazem $k_{2} \geq \epsilon>0>k_{1}$. O resultado segue aplicando o Teorema 2.4.1, deduzimos que $\Sigma$ é homeomorfo a um plano e

$$
\int_{\Sigma} \mid K(I) d A_{I} \leqslant 2 \pi
$$


Por hipótese, temos $K \leq|c|<0$. Daí, podemos estimar a área de $\Sigma$ da seguinte forma:

$$
\int_{\Sigma}|K| d A \geq|c| A(\Sigma)
$$

Portanto, temos que $\frac{2 \pi}{|c|} \geq A(\Sigma)$, o que é absurdo, pois sabemos, pelo Corolário 2.2.1, que qualquer superfície de Hadamard tem área infinita.

Observação 2.4.1. Note que se mudarmos a hipótese $K \leq$ const $<0$ por $K \leq 0$ e $\int_{\Sigma}|K| d A_{I}>$ $2 \pi$ no corolário acima teríamos a mesma conclusão. De fato, basta assumir $k_{2} \leq \epsilon>0$. Então, usando a equação de Gauss da imersão,

$$
k_{1} k_{2}=K-1 \leq-1
$$

$e$ as curvaturas principais de $\psi$ satisfazem a seguinte relação $k_{1} \leq 0<\epsilon \leq k_{2}$. Agora, aplicando o Teorema 2.4.1 temos $\int_{\Sigma}|K| d A_{I} \leq 2 \pi$ o que é um absurdo, pois contraria nossa hipótese.

Agora restringiremos as condições do Corolário 2.4.1 ao exterior de um subconjunto compacto contido na superfície, e usando o Teorema 2.4.2 podemos provar que:

Corolário 2.4.4. Sejam $\psi: \Sigma \longrightarrow \mathbb{H}^{3}$ uma imersão completa e $C \subset \Sigma$ um subconjunto compacto. Suponha que em $\Sigma \backslash C$ a curvatura de $\psi$ é tal que $k \leq-1$ e umas das curvaturas principais satisfaz

$$
k_{i}^{2} \geq \epsilon^{2}>0, i=1,2,
$$

para alguma constante positiva $\epsilon$. Então $\psi$ tem área finita, $\Sigma$ é do tipo parabólico e tem topologia finita.

Demonstração. Mudando a orientação se necessário, e usando a equação de Gauss, podemos assumir que

$$
k_{1} k_{2}=K+1 \leq 0 \quad \text { e } \quad k_{2} \geq \epsilon>0 .
$$

Então $k_{2} \geq \epsilon>0 \geq k_{1}$, isto é, as curvaturas principais são estritamente separadas. Segue do Teorema 2.4.2 que $(\Sigma \backslash C, I)$ tem curvatura total finita. Note que, do fato de $C$ ser compacto, então, sua área e curvatura total são finitas. Agora usando a hipótese da curvatura sobre $\Sigma \backslash C$ temos

$$
A(\Sigma \backslash C) \leq \int_{\Sigma \backslash C}|K| d A_{I}
$$

Logo, podemos dar uma estimativa da área total da superfície, ou seja,

$$
A(\Sigma)=A(\Sigma \backslash C)+A(C) \leq \int_{\Sigma \backslash C}|K| d A_{I}+\int_{C}|K| d A_{I}<\infty .
$$

Portanto a área é finita. Segue do Teorema de Hüber 2.2.2 que a superfície é parabólico e tem topologia finita 
Naturalmente, fazendo um ajuste sobre a curvatura Gauss da imersão no Corolário 2.4.3, podemos obter um resultado em $\mathbb{S}^{3}$ que é análogo ao Corolário 2.4.4.

Corolário 2.4.5. Seja $\psi: \Sigma \longrightarrow \mathbb{S}^{3}$ uma imersão completa e $C \subset \Sigma$ um subconjunto compacto. Suponha que em $\Sigma \backslash C$ a curvatura de Gauss de $\psi$ satisfaz $K \leq$ const $<0$ e umas das curvaturas principais $k_{i}$ satisfaz

$$
k_{i}^{2} \geq \epsilon^{2}>0, \quad i=1,2,
$$

para alguma constante $\epsilon>0$. Então $\psi$ tem área finita, $\Sigma$ é do tipo parabólico e tem topologia finita.

Demonstração. De maneira análoga ao corolário anterior, pela equação de Gauss, assumiremos

$$
k_{1} k_{2}=K-1 \leq-1 \quad \text { e } \quad k_{2} \geq \epsilon>0
$$

Então $k_{2} \geq \epsilon>0 \geq k_{1}$, isto é, as curvaturas principais são estritamente separadas. Pela compacidade de $C$, Teorema 2.4 .2 e usando a hipótese sobre a curvatura em $\Sigma \backslash C$ podemos dar uma estimativa da área total a superfície $\Sigma$. Portanto $A(\Sigma)<\infty$ e pelo Teorema de Hüber 2.2.2 a superfície é parabólico e tem topologia finita.

Como a teoria de pares de Codazzi aparece também no estudo de superfícies imersas em espaços ambientes distintos da formas espaciais, usando os Teoremas 2.4.1 e 2.4 .2 pode-se demonstrar resultados tipo Efimov e tipo Milnor análogos aos vistos nesta seção, mas em contextos diferentes. Por exemplo, podemos ter resultados deste tipo para formas espaciais Lorentziana tridimensional $\mathbb{L}^{3}$, também para superfícies imersas nos espaços tridimensionais De sitter e Anti-De sitter, e para superfícies imersas em um espaço de dimensão $n$ (semi-Riemanniano) com campo de vetores normal $\xi$ unitário e paralelo. 
Neste capítulo, serão estudadas superfícies com curvatura de Gauss constante em $\mathbb{H}^{2} \times \mathbb{R}$ e $\mathbb{S}^{2} \times \mathbb{R}$. Começaremos vendo a definição de espaços produto, como se comporta o tensor curvatura em tais espaços e particularizaremos as equações de Gauss e Codazzi, concluindo que o par fundamental $(I, I I)$ não é par de Codazzi. Baseados nos estudos de Juan Aledo, José Espinar e José Gálvez, em [5], definimos um novo par fundamental na superfície em termos da primeira e segunda formas fundamentais e da diferencial da função altura. Vamos provar que tal par fundamental é um par de Codazzi de curvatura extrínseca constante quando a superfície imersa tem curvatura Gaussiana constante. A partir deste novo par, apresentaremos um Teorema do tipo Liebmann para superfícies completas de curvatura Gaussiana constante em $\mathbb{H}^{2} \times \mathbb{R}$ e $\mathbb{S}^{2} \times \mathbb{R}$ cuja demonstração é baseada na versão abstrata do Teorema de Liebmann e nos resultados dados por Aledo, Espinar e Gálvez em [6] e Espinar [14].

\subsection{Introdução}

Alencar, Do Carmo e Tribuzy em [7] propuseram a seguinte questão: quais são as superfícies fechadas de curvatura Gaussiana constante em $\mathbb{H}^{2} \times \mathbb{R}$ e $\mathbb{S}^{2} \times \mathbb{R}$ ? Esta questão tornou-se natural após a classificação das superfícies com curvatura média constante e gênero zero devido a Abresch e Rosenberg em [1].

Gálvez, Espinar e Aledo, em [5], classificaram as superfícies completas de curvatura Gaussiana contante em $\mathbb{S}^{2} \times \mathbb{R}$ e $\mathbb{H}^{2} \times \mathbb{R}$. Destacaremos que, para $K(I)>0$, existe uma única superfície de revolução completa, a menos de isometria, em $\mathbb{H}^{2} \times \mathbb{R}$ e que para $K(I)>1$, existe uma única superftecie de revolução completa, a menos de isometria em $\mathbb{S}^{2} \times \mathbb{R}$. Como objetivo principal, veremos que é possível utilizar a teoria de pares de Codazzi para mostrar um teorema tipo Liebmann em $\mathbb{H}^{2} \times \mathbb{R}$ e $\mathbb{S}^{2} \times \mathbb{R}$. A parte essencial da prova é mostrar a existência de um forma quadrática holomorfa associada à diferencial de Hopf para cada superfície de curvatura Gaussiana constante. 


\subsection{Resultados Básicos}

De agora em diante trataremos com uma variedade 3-dimensional $\mathbb{M}^{2} \times \mathbb{R}$ dada pelo produto de uma superfície Riemanniana $\mathbb{M}^{2}$, a que chamaremos de base, e a reta real $\mathbb{R}$, chamada fibra.

Consideraremos um caso particular deste tipo de variedade cuja base é superfície simplesmente conexa com curvatura constante diferente de zero que, podemos supor ser \pm 1 . Assim, teremos que quando a curvatura da base é 1 , nossa base é $\mathbb{S}^{2}$, e quando é -1 a base é $\mathbb{H}^{2}$, ou seja,

$$
\mathbb{M}^{2}(\varepsilon)=\left\{\begin{array}{llc}
\mathbb{S}^{2}, & \text { se } & \varepsilon=1 \\
\mathbb{H}^{2}, & \text { se } & \varepsilon=-1
\end{array}\right.
$$

Seja $\mathbb{M}^{2}$ uma superfície Riemanniana, cuja métrica denotaremos por $g_{\kappa}$, onde $\kappa$ é a curvatura de Gauss, e consideraremos a variedade produto $\mathbb{M}^{2} \times \mathbb{R}$. Sejam $\pi$ e $\sigma$ as projeções sobre a base $\mathbb{M}^{2}$, e a fibra $\mathbb{R}$, respectivamente. Então a métrica em $\mathbb{M}^{2} \times \mathbb{R}$ é dada por

$$
\langle,\rangle=d \pi\left(g_{\kappa}\right)+d \sigma\left(d t^{2}\right)
$$

onde $d t^{2}$ é a métrica padrão em $\mathbb{R}$.

Por comodidade omitiremos as projeções e escreveremos $\langle\rangle=,g_{\kappa}+d t^{2}$.

Denotaremos por $\bar{\nabla}, \nabla^{\mathbb{M}^{2}}$ e $\nabla^{\mathbb{R}}$ as conexões de Levi-Civita associadas a $\langle\rangle,, g_{\kappa}$ e $d t^{2}$, respectivamente. Para estudar como atua a conexão do ambiente basta saber como esta atua sobre campos horizontais e verticais. Isto é o que nos diz o seguinte resultado (ver [27], Proposição 56, pág. 89).

Proposição 3.2.1. Se $X, Y \in \mathcal{X}\left(\mathbb{M}^{2}\right)$ e $V, W \in \mathcal{X}(\mathbb{R})$, então

1. $\bar{\nabla}_{X} Y$ é um campo horizontal e $\left(\bar{\nabla}_{X} Y\right)^{h}=\nabla_{X}^{\mathbb{M}^{2}} Y \in \mathcal{X}\left(\mathbb{M}^{2}\right)$;

2. $\bar{\nabla}_{V} W$ é um campo vertical e $\left(\bar{\nabla}_{V} W\right)^{v}=\nabla_{V}^{\mathbb{R}} W \in \mathcal{X}(\mathbb{R})$;

3. $\bar{\nabla}_{V} X=\bar{\nabla}_{X} V=0$.

Agora veremos como se relacionam os tensores de curvatura $\bar{R}, \mathbb{M}^{2} R$ e ${ }^{\mathbb{R}} R$ de $\mathbb{M}^{2} \times \mathbb{R}, \mathbb{M}^{2}$ e $\mathbb{R}$ respectivamente ([27], Corolário 58, pág. 89).

Corolário 3.2.1. Se $X, Y \in \mathcal{X}\left(\mathbb{M}^{2}\right)$ e $V, W \in \mathcal{X}(\mathbb{R})$, então

(i) $\bar{R}(X, Y) Z$ é um campo horizontal e $(\bar{R}(X, Y) Z)^{h}={ }^{\mathbb{M}^{2}} R(X, Y) Z \in \mathcal{X}\left(\mathbb{M}^{2}\right)$;

(ii) $\bar{R}(U, V) W$ é um campo vertical e $(\bar{R}(U, V) W)^{v}={ }^{\mathbb{R}} R(U, V) W \in \mathcal{X}(\mathbb{R})$;

(iii) $\bar{R}$ é zero para qualquer outra escolha de $X, \ldots, W$. 
Decorre do corolário acima, juntamente com o fato de que ${ }^{\mathbb{R}} R=0$ o seguinte resultado:

Corolário 3.2.2. Sejam $X, Y \in \mathcal{X}\left(\mathbb{M}^{2} \times \mathbb{R}\right)$ e denotemos por $X^{h}, Y^{h}$ e $Z^{h} \in \mathcal{X}\left(\mathbb{M}^{2}\right)$ as suas projeçôes horizontais. Então

$$
\bar{R}(X, Y) Z=\mathbb{M}^{2} R\left(X^{h}, Y^{h}\right) Z^{h}
$$

Observação 3.2.1. Chamaremos de slices aos conjuntos da forma $\mathbb{M}^{2}(\varepsilon) \times\left\{t_{0}\right\}$ para algum $t_{0} \in \mathbb{R}$ fixo.

\subsubsection{Equações de compatibilidade}

Nesta seção, obteremos as equaçoes de compatibilidade para superfícies $\Sigma \in \mathbb{M}^{2} \times \mathbb{R}$. Para isso introduziremos algumas notações e observações. Além disso, apresentaremos a equação de Gauss que será fundamental em nossos estudos.

Denotemos por $\mathbb{R}_{k}^{4}, k=0,1$, o espaço vetorial real $\mathbb{R}^{4}$ dotado com coordenadas lineares $\left(x_{1}, x_{2}, x_{3}, x_{4}\right)$ e a métrica $\langle$,$\rangle induzida pela forma quadrática \varepsilon x_{1}^{2}+x_{2}^{2}+x_{3}^{2}+x_{4}^{2}$, onde $\varepsilon=1$ (resp. $\varepsilon=-1)$ se $k=0$ (resp. $k=1$ ).

Sejam $\Sigma$ uma superfície orientável e $\psi: \Sigma \longrightarrow \mathbb{M}^{2}(\varepsilon) \times \mathbb{R}$ imersão com campo de vetores normais unitários $N$. Então, consideraremos sobre $\psi$ a métrica induzida, $\langle$,$\rangle restrita à superfície,$ de $\mathbb{M}^{2} \times \mathbb{R}$, a qual denotaremos por $I$. Sejam $\nabla$ e $R$ a conexão e o tensor de curvatura de $\psi$, respectivamente, e $S$ o endomorfismo de Weingarten de $\Sigma$ associado ao normal unitário, isto é, $S X=-\bar{\nabla}_{X} N, X \in \mathcal{X}(\psi)$. Então $I I(X, Y)=-\langle S X, Y\rangle$ é a segunda forma fundamental da superfície e $K(I)$ a curvatura Gaussiana.

Seja $h(u, v)$ a quarta coordenada da imersão $\psi$, a qual chamaremos de função altura. Assim, temos

$$
\psi(u, v)=\left(x_{1}(u, v), x_{2}(u, v), x_{3}(u, v), h(u, v)\right) \in \mathbb{R}^{4}
$$

tal que

$$
\varepsilon x_{1}^{2}(u, v)+x_{2}^{2}(u, v)+x_{3}^{2}(u, v)=\varepsilon .
$$

E se $\varepsilon=-1$ tem-se $x_{1}>0$ onde $u$ e $v$ são parâmetros locais.

Observação 3.2.2. Se $\eta\left(x_{1}, x_{2}, x_{3}, x_{4}\right)=\left(x_{1}, x_{2}, x_{3}, 0\right) \in \mathbb{R}_{k}^{4}$, então $\eta$ é o vetor normal unitário de $\mathbb{M}^{2} \times \mathbb{R}$ em $\mathbb{R}_{k}^{4}$. De fato, seja $\left\{\psi_{u}(u, v), \psi_{v}(u, v)\right\}$ uma base do plano tangente $T_{q} \psi$ em $q=$ $\psi(u, v)$. Derivando (3.1) em relação a u temos que

$$
2 \varepsilon x_{1} \frac{\partial x_{1}}{\partial u}+2 x_{2} \frac{\partial x_{2}}{\partial u}+2 x_{3} \frac{\partial x_{3}}{\partial u}=0,
$$

ou seja, $2\left\langle\psi_{u}, \eta\right\rangle=0$. De modo análogo, temos que $2\left\langle\psi_{v}, \eta\right\rangle=0$. Logo $\eta \in\left(T_{q} \psi\right)^{\perp}$ e $|\eta|=1$. Como $\eta$ é normal a $N$, então segue nossa afirmação. 
Observação 3.2.3. Observe que $\langle-d \eta, d \psi\rangle=\langle-d \psi, d \psi\rangle+d h^{2}$. De fato, como $\langle-d \eta, d \psi\rangle=$ $-d x_{1}^{2}-d x_{2}^{2}-d x_{3}^{2}$ e $\langle-d \psi, d \psi\rangle=-d x_{1}^{2}-d x_{2}^{2}-d x_{3}^{2}-d h^{2}$, segue que $\langle-d \psi, d \psi\rangle=\langle-d \eta, d \psi\rangle-d h^{2}$.

Denotemos por:

(1) $T$ a projeção do vetor vertical $\partial_{t}$ sobre o espaço tangente de $\Sigma$;

(2) $\nu=\left\langle N, \partial_{t}\right\rangle$, a componente normal de $N$. A $\nu$ chamaremos função ângulo da superfície.

Notemos $T=\nabla h$, isto é, $T$ é igual ao gradiente da função altura $h$, onde $\nabla h=\left.\sigma\right|_{\Sigma}: \Sigma \longrightarrow$ $T_{p} \Sigma$. Com efeito, como $T \in \mathfrak{X}(\Sigma)$, temos $T=a \partial_{u}+b \partial_{v}$, para algumas funções $a$ e $b$ reais. Agora escrevemos o campo $\partial_{t}$ em sua parte tangente e normal, isto é, $\partial_{t}=T+\lambda N \Rightarrow \lambda=\left\langle\partial_{t}, N\right\rangle=\nu$. Logo,

$$
\partial_{t}=T+\nu N
$$

Fazendo o produto interno de (3.2), com $\partial_{u}$ e $\partial_{v}$, obtemos

$$
\left\langle\partial_{t}, \partial_{u}\right\rangle=\left\langle a \partial_{u}+b \partial_{v}+\nu N, \partial_{u}\right\rangle=a\left\langle\partial_{u}, \partial_{u}\right\rangle+b\left\langle\partial_{v}, \partial_{u}\right\rangle=a E+b F=h_{u}
$$

Analogamente $\left\langle\partial_{t}, \partial_{v}\right\rangle=a F+b G=h_{v}$, donde segue que

$$
a=\frac{G h_{u}-F h_{v}}{E G-F^{2}} \quad \text { e } \quad b=\frac{-F h_{u}-E h_{v}}{E G-F^{2}} .
$$

Portanto,

$$
T=\frac{G h_{u}-F h_{v}}{E G-F^{2}} \partial_{u}+\frac{-F h_{u}-E h_{v}}{E G-F^{2}} \partial_{v}=\nabla h .
$$

Proposição 3.2.2. Sejam $\Sigma$ uma superfície orientável e $\psi: \Sigma \longrightarrow \mathbb{M}^{2}(\varepsilon) \times \mathbb{R}$ uma imersão com campo normal unitário $N$ e operador forma associado $S$. Denotaremos por $h$ a função altura em $\Sigma$, por $\nu$ a quarta coordenada do normal unitário $N$ e por $T$ a projeção do vetor vertical $\partial_{t}$ sobre o espaço tangente de $\Sigma$. Então as seguintes equaçôes são satisfeitas

$$
\begin{array}{rlrl}
K(I) & =K+\varepsilon \nu^{2}, & & \text { Gauss } \\
\nabla_{X} S Y-\nabla_{Y} S X-S[X, Y] & =\varepsilon \nu(\langle Y, T\rangle X-\langle X, T\rangle Y), & & \text { Codazzi } \\
\nabla_{X} T & =\nu S X, & \\
d \nu(X) & =-\langle S X, T\rangle, & \\
\|T\|^{2}+\nu^{2} & =1 . &
\end{array}
$$

Demonstração. Segue de (3.2)

$$
1=\left\langle\partial_{t}, \partial_{t}\right\rangle=\langle T+\nu N, T+\nu N\rangle=\|T\|^{2}+\nu^{2}
$$


onde fica demonstrado (3.7).

Agora notemos que $\partial_{t}$ é um campo paralelo, logo $\bar{\nabla}_{X} \partial_{t}=0$. Assim, de (3.2), obtemos

$$
0=\bar{\nabla}_{X} T+(d \nu(X)) N+\nu \bar{\nabla}_{X} N
$$

Podemos escrever

$$
\bar{\nabla}_{X} T=\left(\bar{\nabla}_{X} T\right)^{t}+c N
$$

donde $\left\langle\bar{\nabla}_{X} T, N\right\rangle=c$. Como $\langle T, N\rangle=0$, então

$$
\left\langle\bar{\nabla}_{X} T, N\right\rangle+\left\langle\bar{\nabla}_{X} N, T\right\rangle=0
$$

$\log 0$

$$
\left\langle\bar{\nabla}_{X} T, N\right\rangle=-\left\langle\bar{\nabla}_{X} N, T\right\rangle=\langle T, A X\rangle .
$$

Segue de (3.8)

$$
0=\nabla_{X} T+\langle A X, T\rangle N+(d \nu(X)) N-\nu A X
$$

Tomando a parte tangente e a parte normal nesta igualdade, temos

$$
\nabla_{X} T=\nu S X \quad \text { e } \quad d \nu(X)=-\langle S X, T\rangle
$$

na qual ficam demonstradas as equações (3.5) e (3.6).

Dados $X, Y, Z \in \mathcal{X}(\psi)$, as equações de Gauss e Codazzi de uma superfície em um espaço tridimensional são dadas, respectivamente, por

$$
\begin{array}{r}
R(X, Y) Z-\bar{R}(X, Y) Z=\langle S X, Z\rangle S Y-\langle S Y, Z\rangle S X, \\
\bar{R}(X, Y) N=\nabla_{X} S Y-\nabla_{Y} S X-S[X, Y] .
\end{array}
$$

Segue do Corolário 3.2.2 que $\bar{R}(X, Y) Z={ }^{\mathbb{M}^{2}} R\left(X^{h}, Y^{h}\right) Z^{h}$. Além disso, expressando

$$
\mathbb{M}^{2} R\left(X^{h}, Y^{h}\right) Z^{h}=\varepsilon\left(\left\langle X^{h}, Z^{h}\right\rangle Y^{h}-\left\langle Y^{h}, Z^{h}\right\rangle X^{h}\right),
$$

onde $\varepsilon$ é a curvatura de Gauss de $\mathbb{M}^{2}$. Podemos escrever, $X=X^{h}+X^{v}$, tal que $X \in \mathbb{M}^{\in} \times \mathbb{R}$. Prosseguindo, rescreveremos a igualdade acima da seguinte forma: $X=X^{h}+c \partial_{t}$, tal que $c$ é alguma função real. Fazendo o produto interno na equação anterior por $\partial_{t}$, e observando que $\left\langle X^{h}, \partial_{t}\right\rangle=0$, obtemos que $c=\left\langle X, \partial_{t}\right\rangle$, assim $X^{h}=X-\left\langle X, \partial_{t}\right\rangle \partial_{t}$. Portanto para quaisquer $X$, $Y, Z \in \mathcal{X}\left(\mathbb{M}^{2} \times \mathbb{R}\right)$ 


$$
\begin{aligned}
\bar{R}(X, Y) Z= & \mathbb{M}^{2} R\left(X^{h}, Y^{h}\right) Z^{h} \\
= & \varepsilon\left(\left\langle X^{h}, Z^{h}\right\rangle Y^{h}-\left\langle Y^{h}, Z^{h}\right\rangle X^{h}\right) \\
= & \varepsilon\left(\left(\langle X, Z\rangle-\left\langle X, \partial_{t}\right\rangle\left\langle Z, \partial_{t}\right\rangle\right)\left(Y-\left\langle Y, \partial_{t}\right\rangle \partial_{t}\right)\right. \\
& \left.-\left(\langle Y, Z\rangle-\left\langle Z, \partial_{t}\right\rangle\left\langle Y, \partial_{t}\right\rangle\right)\left(X-\left\langle X, \partial_{t}\right\rangle \partial_{t}\right)\right) \\
= & \varepsilon\left(\langle X, Z\rangle Y-\langle Y, Z\rangle X-\left\langle X, \partial_{t}\right\rangle\left\langle Z, \partial_{t}\right\rangle Y\right. \\
& \left.+\left\langle Z, \partial_{t}\right\rangle\left\langle Y, \partial_{t}\right\rangle X+\langle Y, Z\rangle\left\langle X, \partial_{t}\right\rangle-\left\langle Y, \partial_{t}\right\rangle\langle X, Z\rangle \partial_{t}\right) .
\end{aligned}
$$

Isto é,

$$
\begin{aligned}
& \bar{R}(X, Y) Z=\varepsilon\left(\langle X, Z\rangle Y-\langle Y, Z\rangle X-\left\langle X, \partial_{t}\right\rangle\left\langle Z, \partial_{t}\right\rangle Y\right. \\
& \left.\quad+\left\langle Z, \partial_{t}\right\rangle\left\langle Y, \partial_{t}\right\rangle X+\langle Y, Z\rangle\left\langle X, \partial_{t}\right\rangle-\left\langle Y, \partial_{t}\right\rangle\langle X, Z\rangle \partial_{t}\right) .
\end{aligned}
$$

Tomando $Z=N, X$ e $Y \in \mathcal{X}(\psi)$ temos

$$
\begin{gathered}
\bar{R}(X, Y) N=\varepsilon\left(\left\langle N, \partial_{t}\right\rangle\left(\left\langle\partial_{t}, Y\right\rangle X-\left\langle\partial_{t}, X\right\rangle Y\right)\right. \\
\bar{R}(X, Y) N=\varepsilon \nu(\langle T, Y\rangle X-\langle T, X\rangle Y)
\end{gathered}
$$

e por (3.10), temos que a equação de Codazzi se verifica.

Para obter a equação de Gauss em $\mathbb{M}^{2} \times \mathbb{R}$ multiplicaremos por um campo $W$ a equação (3.9). Assim, de (3.11)

$$
\begin{aligned}
\langle\bar{R}(X, Y) Z, W\rangle= & \varepsilon(\langle X, Z\rangle\langle Y, W\rangle-\langle Y, Z\rangle\langle X, W\rangle \\
& -\left\langle X, \partial_{t}\right\rangle\left\langle Z, \partial_{t}\right\rangle\langle Y, W\rangle+\left\langle Z, \partial_{t}\right\rangle\left\langle Y, \partial_{t}\right\rangle\langle X, W\rangle \\
& +\left(\langle Y, Z\rangle\left\langle X, \partial_{t}\right\rangle-\left\langle Y, \partial_{t}\right\rangle\langle X, Z\rangle\left\langle\partial_{t}, W\right\rangle\right) .
\end{aligned}
$$

Tomando $X, Y \in \mathcal{X}(\psi)$ uma base ortonormal e fazendo $Z=X, W=Y$, (3.12) fica

$$
\begin{aligned}
\langle\bar{R}(X, Y) Z, W\rangle & =\varepsilon\left(1-\left\langle X, \partial_{t}\right\rangle^{2}-\left\langle Y, \partial_{t}\right\rangle^{2}\right) \\
& =\varepsilon\left(1-\langle X, T\rangle^{2}-\langle Y, T\rangle^{2}\right) \\
& =\varepsilon\left(1-\|T\|^{2}\right) \\
& =\varepsilon \nu^{2},
\end{aligned}
$$

$\log 0$

$$
\langle\bar{R}(X, Y) X, Y\rangle=\varepsilon \nu^{2} .
$$


Observemos que, dados $X, Y \in \mathcal{X}(\psi)$ base ortonormal, temos

$$
\langle R(X, Y) X, Y\rangle=K(I)
$$

e

$$
K=\operatorname{det} S=\langle S X, X\rangle\langle S Y, Y\rangle-\langle S Y, W\rangle\langle S Y, X\rangle .
$$

Portanto

$$
K(I)-\varepsilon \nu^{2}=K(I, I I)
$$

isto é

$$
K(I)=K(I, I I)+\varepsilon \nu^{2},
$$

como queríamos.

Observação 3.2.4. Notemos que da Definição 1.3 .1 e a equação (3.4) temos que o Tensor de Codazzi pode ser escrito como: $T_{S}(X, Y)=\varepsilon \nu(\langle Y, T\rangle X-\langle X, T\rangle Y)$. Ou seja, como $T_{S}$ não se anula, então $(I, I I)$ não é par de Codazzi em $\mathbb{M}^{2}(\varepsilon) \times \mathbb{R}$.

\subsection{Superfícies de Revolução Completas de Curvatura Constante}

Nesta seção descreveremos brevemente as superfícies de revolução completas com curvaturas Gaussiana constante $K(I)$ em $\mathbb{M}^{2}(\varepsilon) \times \mathbb{R}$.

Como as rotações preservam orientação e o eixo de rotação, podemos escolher a menos de isometria, o eixo de rotação sendo $(1,0,0) \times \mathbb{R}$, e o grupo de rotação dada por

$$
M=\left(\begin{array}{cccc}
1 & 0 & 0 & 0 \\
0 & \cos v & -\sin v & 0 \\
0 & \sin v & \cos v & 0 \\
0 & 0 & 0 & 1
\end{array}\right)
$$

onde $v$ é o ângulo de rotação.

Notemos que o conjunto

$$
\mathcal{P}=\left\{\left(x_{1}, \ldots, x_{4}\right) \in \mathbb{M}^{2}(\varepsilon) \times \mathbb{R} ; x_{2} \geq 0 \quad \text { e } x_{3}=0\right\}
$$

intercepta toda órbita da rotação uma única vez. Assim, iremos tomar uma curva $\alpha$ contida em $\mathcal{P}$ tal que $\alpha$ não toque o eixo de rotação exceto no ponto inicial e final. Como queremos superfícies completas, se a curva intercepta o eixo de rotação, em pontos iniciais e finais, deverá ser ortogonalmente.

Apresentaremos agora superfície rotacionalmente invariante em $\mathbb{M}^{2}(\varepsilon) \times \mathbb{R}$ com curvatura constante $K$ positiva. Para mais detalhes, veja [5] e [6]. 
Proposição 3.3.1. Seja $\Sigma$ uma superfície invariante (em torno do eixo $\{1,0,0\} \times \mathbb{R}$ ) em $\mathbb{H}^{2} \times \mathbb{R}$ com curvatura Gaussiana $K$ constante positiva, dada por

$$
\psi(u, v)=(\cosh l(u), \sinh l(u) \cos v, \sinh l(u) \sin v, h(u))
$$

gerada pela curva $\alpha(u)=(\cosh l(u), \sin l(u), 0, h(u))$, onde u é o parâmetro comprimento de arco ao longo de $\alpha$, isto é, $l^{\prime}(u)^{2}+h^{\prime}(u)^{2}=1$. Então $\Sigma$ deve ser imersa e $\alpha$ é dada por

$$
\begin{aligned}
& l(u)=\arcsin \left(\frac{1}{\sqrt{K}} \sin (\sqrt{K} u)\right) \\
& h(u)=-\sqrt{\frac{1+K}{K}} \arctan \left(\frac{\cos \sqrt{K} u}{\sqrt{K+\sin ^{2}(\sqrt{K} u)}}\right),
\end{aligned}
$$

onde $0 \leq u \leq \frac{\pi}{\sqrt{K}}$. Ademais, $\psi$ é, a menos de isometrias, a parametrização da única superfície completa com curvatura Gaussiana constate $K$ em $\mathbb{H}^{2} \times \mathbb{R}$. Particularmente, $\Sigma$ deve ser uma esfera topológica.

Proposição 3.3.2. Seja $\Sigma$ uma superfície invariante (em torno do eixo $\{1,0,0\} \times \mathbb{R}$ ) em $\mathbb{S}^{2} \times \mathbb{R}$ com curvatura Gaussiana $K>1$ constante, dada por

$$
\psi(u, v)=(\sin l(u), \cos l(u) \cos v, \cos l(u) \sin v, h(u))
$$

gerada pela curva $\alpha(u)=(\cos l(u), \sin l(u), 0, h(u))$. Então $\Sigma$ deve ser imersa e $\alpha$ é dada por

$$
\begin{aligned}
l(u) & =\arccos \left(\frac{1}{\sqrt{K}} \sin (\sqrt{K} u)\right) \\
h(u) & =-\sqrt{\frac{K-1}{K}} \log \left(\frac{\cos (\sqrt{K} u)+\sqrt{K-\sin ^{2}(\sqrt{K} u)}}{1+\sqrt{K}}\right)
\end{aligned}
$$

onde $0 \leq u \leq \frac{\pi}{\sqrt{K}}$. Ademais, $\psi$ é, a menos de isometrias, a parametrização da única superfície completa com curvatura Gaussiana constante $K$ em $\mathbb{S}^{2} \times \mathbb{R}$. Particularmente, $\Sigma$ deve ser uma esfera topológica.

\subsection{Par de Codazzi para Superfícies de Curvatura Constante em $\mathbb{H}^{2} \times \mathbb{R}$ e $\mathbb{S}^{2} \times \mathbb{R}$}

Já vimos que o par $(I, I I)$ não define um par de Codazzi em superfícies de $\mathbb{M}^{2} \times \mathbb{R}$. Ao longo deste capítulo, consideraremos $K \neq \varepsilon$ e então podemos definir uma nova quadrática $A$, dada por

$$
A=I+\frac{1}{\varepsilon K(I)-1} d h^{2}
$$


em que $I$ é a primeira forma fundamental e $h$ é a função altura. Nosso objetivo nesta seção é estabelecer condições para que o par $(A, I I)$ seja um par de Codazzi de curvatura extrínseca constante.

Se $A$ é uma métrica Riemanniana podemos relacionar a curvatura extrínseca $K(A, I I)$ com a curvatura Gaussiana $K(I)$

Lema 3.4.1. Se A é uma métrica Riemanniana em $\Sigma$, então a curvatura extrínseca do par $(A, I I)$ é dada por

$$
K(A, I I)=K(I)-\varepsilon
$$

Demonstração. Sejam $(u, v)$ parâmetros isotérmicos sobre $\Sigma$ para $I$, isto é

$$
I=E\left(d u^{2}+d v^{2}\right)
$$

Tome $c=\frac{1}{\varepsilon K(I)-1}$, então temos que

$$
\begin{aligned}
& A=I+c d h^{2} \\
& A=E\left(d u^{2}+d v^{2}\right)+c\left(h_{u} d u+h_{v} d v\right)^{2} \\
& A=E\left(d u^{2}+d v^{2}\right)+c\left(h_{u}^{2} d u^{2}+2 h_{u} h_{v} d u d v+h_{v}^{2} d v^{2}\right) \\
& A=\left(E+c h_{u}^{2}\right) d u^{2}+2 c h_{u} h_{v} d u d v+\left(E+c h_{v}^{2}\right) d v^{2} .
\end{aligned}
$$

Assim, os coeficientes de $A$ são dado por:

$$
\begin{aligned}
& \bar{E}=E+c h_{u}^{2} \\
& \bar{F}=c h_{u} h_{v} \\
& \bar{G}=E+c h_{v}^{2} .
\end{aligned}
$$

Logo, podemos reescrever $A$ como: $A=\bar{E} d u^{2}+2 \bar{F} d u d v+\bar{G} d v^{2}$. Daí,

$$
\begin{aligned}
K(A, I I) & =\frac{e g-f^{2}}{\left(E+c h_{u}^{2}\right)\left(E+c h_{v}^{2}\right)-\left(c h_{u} h_{v}\right)^{2}} \\
& =\frac{e g-f^{2}}{E^{2}+E c h_{v}^{2}+E c h_{u}^{2}} \\
& =\frac{e g-f^{2}}{E^{2}\left(1+c\left(\frac{h_{u}^{2}+h_{v}^{2}}{E}\right)\right)} \\
& =\frac{e g-f^{2}}{E^{2}}\left(\frac{1}{1+c\|\nabla h\|^{2}}\right) \\
& =\frac{K(I, I I)}{1+c\|\nabla h\|^{2}}
\end{aligned}
$$

Substituindo $c$ pelo seu valor temos 


$$
K(A, I I)=\frac{(\varepsilon K(I)-1) K(I, I I)}{\varepsilon K(I)-1+\|\nabla h\|^{2}}
$$

Sabemos que a equação de Gauss é dada por $K(I)=K(I, I I)+\varepsilon \nu^{2}$ tal que $\nu^{2}=1-\|\nabla h\|^{2}$. Segue daí que $K(I, I I)=K(I)-\varepsilon \nu^{2}$, e então

$$
\begin{aligned}
K(A, I I) & =\frac{\left(K(I)-\varepsilon \nu^{2}\right)(\varepsilon K(I)-1)}{\varepsilon K(I)-\nu^{2}} \\
& =\frac{\varepsilon K(I)^{2}-K(I)-\nu^{2} K(I)+\varepsilon \nu^{2}}{\varepsilon K(I)-\nu^{2}} \\
& =\frac{\left(\varepsilon K(I)-\nu^{2}\right)(K(I)-\varepsilon)}{\varepsilon K(I)-\nu^{2}} \\
& =K(I)-\varepsilon .
\end{aligned}
$$

Observe que, no lema anterior, $A$ é Riemanniana se, e somente se, $1+c\|\nabla h\|^{2}>0$. De fato, se $A$ uma métrica Riemanniana, então $0<\overline{E G}-\bar{F}^{2}$. Portanto

$$
0<\left(E+c h_{u}^{2}\right)\left(E+c h_{v}^{2}\right)-\left(c h_{u} h_{v}\right)^{2}=E^{2}\left(1+c\|\nabla h\|^{2}\right)
$$

ou seja $1+c\|\nabla h\|^{2}>0$. Reciprocamente, se $1+c\|\nabla h\|^{2}>0$, então

$$
\begin{gathered}
\left(E+c h_{u}^{2}\right)+\left(E+c h_{v}^{2}\right)=E\left(2+c\|\nabla h\|^{2}\right)>0 \\
\left(E+c h_{u}^{2}\right)\left(E+c h_{v}^{2}\right)-\left(c h_{u} h_{v}\right)^{2}=E^{2}\left(1+c\|\nabla h\|^{2}\right)>0 .
\end{gathered}
$$

Segue das igualdades acima que $A$ é positiva definida, portanto, $A$ é uma métrica Riemanniana. Como consequência obtemos

Lema 3.4.2. Seja $\Sigma$ uma superfície orientável e seja $\psi: \Sigma \longrightarrow \mathbb{M}^{2}(\varepsilon) \times \mathbb{R}$ uma imersão com curvatura Gaussiana $K(I)$. Então a forma quadrática A não é uma métrica Riemanniana em $\Sigma$ se, e somente se,

(a) existe $p \in \Sigma$ satisfazendo $0 \leq K(I) \leq 1$ e $\|\nabla h\|^{2} \geq 1-K(I)$, quando $\varepsilon=1$;

(b) existe $p \in \Sigma$ satisfazendo $-1 \leq K(I) \leq 0$ e $\|\nabla h\|^{2} \geq 1+K(I)$, quando $\varepsilon=-1$, tal que h é a função altura da imersão $\psi$.

Demonstração. Note que $1+c\|\nabla h\|^{2}>0$ não é satisfeita se, e somente se, $c \leq-1$ e $\|\nabla h\|^{2} \geq-\frac{1}{c}$. De fato, se $c \leq-1$ e $\|\nabla h\|^{2} \geq-\frac{1}{c}$, então $c \leq 0$ e $c\|\nabla h\|^{2} \leq-1$. Portanto $c\|\nabla h\|^{2}+1 \leq 0$. Reciprocamente, se $1+c\|\nabla h\|^{2} \leq 0$, temos que $c<0$ e então $\|\nabla h\|^{2} \geq \frac{-1}{c}$. Como $\|\nabla h\|^{2} \leq 1$ temos $1 \geq \frac{-1}{c}$, o que implica $c \leq-1$. 
a) Se $\varepsilon=1$ temos que $c=\frac{1}{K(I)-1}$. Logo $1+c\|\nabla h\|^{2} \leq 0$ se, e somente se,

$$
\frac{1}{K(I)-1} \leq-1 \quad \text { e } \quad\|\nabla h\|^{2} \geq 1-K(I)
$$

Isto é,

$$
0 \leq K(I)<1 \quad \text { e } \quad\|\nabla h\|^{2} \geq 1-K(I)
$$

b) Se $\varepsilon=-1$ temos que $c=\frac{1}{-K(I)-1}$. Logo $1+c\|\nabla h\|^{2} \leq 0$ se, e somente se,

$$
\frac{1}{-K(I)-1} \leq-1 \quad \text { e } \quad\|\nabla h\|^{2} \geq 1+K(I)
$$

Isto é,

$$
-1<K(I) \leq 0 \quad \text { e } \quad\|\nabla h\|^{2} \geq 1+K(I) .
$$

Agora focaremos nossas atenções nas imersões $\psi: \Sigma \longrightarrow \mathbb{M}^{2}(\varepsilon) \times \mathbb{R}$ com curvatura Gaussiana $K(I)$ constante.

Lema 3.4.3. Para todo ponto $p \in \Sigma$, existe uma parametrização $X(u, v)$ de uma vizinhança de p e um domínio $U \subseteq \mathbb{R}^{2}$ tal que a métrica induzida é dada por

$$
I=\frac{1}{\tau}\left(d u^{2}+d v^{2}\right), \quad \tau=\frac{1}{2}\left(1+K(I)\left(u^{2}+v^{2}\right)\right) .
$$

Demonstração. Como $X$ é uma parametrização ortogonal, isto é, $F=0$, temos

$$
K(I)=-\frac{1}{2 \sqrt{E G}}\left\{\left(\frac{E_{v}}{\sqrt{E G}}\right)_{v}+\left(\frac{G_{u}}{\sqrt{E G}}\right)_{u}\right\} .
$$

Fazendo $E=G=\frac{1}{\tau^{2}}=\lambda$ temos que

$$
\begin{aligned}
& K(I)=-\frac{1}{2 \lambda}\left\{\left(\frac{\lambda_{v}}{\lambda}\right)_{v}+\left(\frac{\lambda_{u}}{\lambda}\right)_{u}\right\} \\
& K(I)=-\frac{1}{2 \lambda}\left\{(\log \lambda)_{v v}+(\log \lambda)_{u u}\right\} .
\end{aligned}
$$


Notemos

$$
\begin{aligned}
& (\log \lambda)_{v v}=\left(\log \frac{1}{\tau^{2}}\right)_{v}=\left(-\frac{2 \tau_{v}}{\tau}\right)_{v}=-\frac{2 \tau_{v v}}{\tau}+\frac{2 \tau_{v}^{2}}{\tau^{2}} \\
& (\log \lambda)_{u u}=\left(\log \frac{1}{\tau^{2}}\right)_{u}=\left(-\frac{2 \tau_{u}}{\tau}\right)_{u}=-\frac{2 \tau_{u u}}{\tau}+\frac{2 \tau_{u}^{2}}{\tau^{2}}
\end{aligned}
$$

Logo,

$$
\begin{aligned}
& K(I)=-\frac{1}{\frac{2}{\tau^{2}}}\left\{-\frac{2 \tau_{v v}}{\tau}+\frac{2 \tau_{v}^{2}}{\tau^{2}}-\frac{2 \tau_{u u}}{\tau}+\frac{2 \tau_{u}^{2}}{\tau^{2}}\right\} \\
& K(I)=-\tau^{2}\left\{\frac{1}{\tau^{2}}\left(\tau_{v}^{2}+\tau_{u}^{2}\right)+\frac{1}{\tau}\left(-\tau_{v v}-\tau_{u u}\right)\right\} \\
& K(I)=-\left(\tau_{v}^{2}+\tau_{u}^{2}\right)+\tau\left(\tau_{v v}+\tau_{u u}\right) .
\end{aligned}
$$

Segue que, se $\tau=\frac{1}{2}\left(1+K(I)\left(u^{2}+v^{2}\right)\right)$, então $I$ é uma métrica de curvatura constante $K(I)$. Como consideramos uma imersão $\psi$ com curvatura Gaussiana $K(I)$ constante, segue do Teorema de Cartan* nosso resultado.

O lema a seguir fornece as equações de Codazzi de $\Sigma$ em $\mathbb{M}^{2} \times \mathbb{R}$.

Lema 3.4.4. Considere uma imersão $\psi: \Sigma \longrightarrow \mathbb{M}^{2}(\varepsilon) \times \mathbb{R}$ com curvatura Gaussiana $K(I)$ constante e $(X, U)$ uma parametrização isotérmica para a métrica induzida $I$, em $\Sigma$, tal que

$$
\begin{aligned}
I & =\frac{1}{\tau}\left(d u^{2}+d v^{2}\right) \\
I I & =e d u^{2}+2 f d u d v+g d v^{2},
\end{aligned}
$$

onde $\tau(u, v)=\frac{1}{2}\left(1+K(I)\left(u^{2}+v^{2}\right)\right)$. Ent $\tilde{a} o$

$$
\begin{aligned}
& \psi_{u u}=\left(-\frac{K(I) u}{\tau}\right) \psi_{u}+\left(\frac{K(I) v}{\tau}\right) \psi_{v}+e N+\varepsilon\left(h_{u}^{2}-\frac{1}{\tau^{2}}\right) \eta \\
& \psi_{u v}=\left(-\frac{K(I) v}{\tau}\right) \psi_{u}+\left(-\frac{K(I) u}{\tau}\right) \psi_{v}+f N+\varepsilon\left(h_{u} h_{v}\right) \eta \\
& \psi_{v v}=\left(\frac{K(I) u}{\tau}\right) \psi_{u}+\left(-\frac{K(I) v}{\tau}\right) \psi_{v}+g N+\varepsilon\left(h_{v}^{2}-\frac{1}{\tau^{2}}\right) \eta .
\end{aligned}
$$

*Ver [9], capítulo $V I I$, seção 2 
Consequentemente,

$$
\begin{array}{rlc}
K(I) & =\left(e g-f^{2}\right) \tau^{4}+\varepsilon\left(1-\|\nabla h\|^{2}\right) & \text { Gauss } \\
e_{v}-f_{u} & =-\frac{K(I) u}{\tau}(e+g)-\varepsilon \frac{\nu h_{v}}{\tau^{2}} & \text { Codazzi }(I) \\
f_{v}-g_{u} & =-\frac{K(I) u}{\tau}(e+g)+\varepsilon \frac{\nu h_{u}}{\tau^{2}} & \text { Codazzi }(I I),
\end{array}
$$

Demonstração. Temos que $\left\{\psi_{u}, \psi_{v}, N, \eta\right\}$ formam uma base ortonormal para $\mathbb{R}_{k}^{4}$. Assim podemos escrever

$$
\begin{aligned}
& \psi_{u u}=\Gamma_{11}^{1} \psi_{u}+\Gamma_{11}^{2} \psi_{v}+a_{1} N+\bar{a}_{1} \eta \\
& \psi_{u v}=\Gamma_{12}^{1} \psi_{u}+\Gamma_{12}^{2} \psi_{v}+a_{2} N+\bar{a}_{2} \eta \\
& \psi_{v v}=\Gamma_{22}^{1} \psi_{u}+\Gamma_{22}^{2} \psi_{v}+a_{3} N+\bar{a}_{3} \eta
\end{aligned}
$$

Notemos que

$$
\begin{aligned}
& \left\langle\psi_{u u}, N\right\rangle=a_{1}=e, \\
& \left\langle\psi_{u v}, N\right\rangle=a_{2}=f, \\
& \left\langle\psi_{v v}, N\right\rangle=a_{3}=g,
\end{aligned}
$$

e

$$
\begin{aligned}
& \bar{e}=\left\langle\psi_{u u}, \eta\right\rangle=\varepsilon \bar{a}_{1}, \\
& \bar{f}=\left\langle\psi_{u v}, \eta\right\rangle=\varepsilon \bar{a}_{2}, \\
& \bar{g}=\left\langle\psi_{v v}, \eta\right\rangle=\varepsilon \bar{a}_{3} .
\end{aligned}
$$

Logo

$$
\begin{aligned}
& \psi_{u u}=\Gamma_{11}^{1} \psi_{u}+\Gamma_{11}^{2} \psi_{v}+e N+\varepsilon \bar{e} \eta \\
& \psi_{u v}=\Gamma_{12}^{1} \psi_{u}+\Gamma_{12}^{2} \psi_{v}+f N+\varepsilon \bar{f} \eta \\
& \psi_{v v}=\Gamma_{22}^{1} \psi_{u}+\Gamma_{22}^{2} \psi_{v}+g N+\varepsilon \bar{g} \eta
\end{aligned}
$$

Se $\langle-d \eta, d \psi\rangle=\langle-d \psi, d \psi\rangle+d h^{2}$, ver Observação 3.2.3, então

$$
\begin{aligned}
& \langle-d \eta, d \psi\rangle=\left\langle-\eta_{u} d u-\eta_{v} d v, \psi_{u} d u+\psi_{v} d v\right\rangle= \\
& =\left\langle-\eta_{u}, \psi_{u}\right\rangle d u^{2}+\left\langle-\eta_{u}, \psi_{v}\right\rangle d u d v+\left\langle-\eta_{v}, \psi_{u}\right\rangle d u d v+\left\langle-\eta_{v}, \psi_{v}\right\rangle d v^{2}
\end{aligned}
$$


Como $\left\langle\eta, \psi_{u}\right\rangle=\left\langle\eta, \psi_{v}\right\rangle=0$, segue que

$$
\begin{aligned}
\left\langle-\eta_{u}, \psi_{u}\right\rangle & =\bar{e} \\
\left\langle-\eta_{v}, \psi_{u}\right\rangle & =\left\langle-\eta_{u}, \psi_{v}\right\rangle=\bar{f}, \\
\left\langle-\eta_{v}, \psi_{v}\right\rangle & =\bar{g} .
\end{aligned}
$$

Daí $\langle-d \eta, d \psi\rangle=\left\langle\eta, \psi_{u u}\right\rangle d u^{2}+\left\langle\eta, \psi_{u v}\right\rangle d u d v+\left\langle\eta, \psi_{v v}\right\rangle d v^{2}$. Por outro lado,

$$
\begin{aligned}
& \langle-d \psi, d \psi\rangle+d h^{2}= \\
& =\left\langle-\psi_{u} d u-\psi_{v} d v, \psi_{u} d u+\psi_{v} d v\right\rangle+\left(h_{u} d u+h_{v} d v\right)^{2} \\
& =-\left\langle\psi_{u}, \psi_{u}\right\rangle d u^{2}-\left\langle\psi_{v}, \psi_{v}\right\rangle d v^{2}+h_{u}^{2} d u^{2}+2 h_{u} h_{v} d u d v+h_{v}^{2} d v^{2} \\
& =\left(-\left\langle\psi_{u}, \psi_{u}\right\rangle+h_{u}^{2}\right) d u^{2}+2 h_{u} h_{v} d u d v+\left(-\left\langle\psi_{v}, \psi_{v}+h_{v}^{2}\right) d v^{2}\right.
\end{aligned}
$$

Igualando cada membro, temos

$$
\begin{aligned}
& \left\langle\eta, \psi_{u u}\right\rangle=-\left\langle\psi_{u}, \psi_{u}\right\rangle+h_{u}^{2}=-E+h_{u}^{2}=h_{u}^{2}-\frac{1}{\tau^{2}} \\
& \left\langle\eta, \psi_{u v}\right\rangle=h_{u} h_{v} \\
& \left\langle\eta, \psi_{v v}\right\rangle=-\left\langle\psi_{v}, \psi_{v}\right\rangle+h_{v}^{2}=-G+h_{v}^{2}=h_{v}^{2}-\frac{1}{\tau^{2}} .
\end{aligned}
$$

Logo,

$$
\begin{aligned}
\bar{e} & =h_{u}^{2}-\frac{1}{\tau^{2}}, \\
\bar{f} & =h_{u} h_{v} \\
\bar{g} & =h_{v}^{2}-\frac{1}{\tau^{2}}
\end{aligned}
$$

Agora analisando os símbolos de Christoffel em relação à métrica $I$, temos

$$
\begin{gathered}
\Gamma_{11}^{1}=\frac{E_{u}}{2 E}=\frac{\left(\frac{1}{\tau^{2}}\right)_{u}}{\frac{2}{\tau^{2}}}=-\frac{\tau_{u}}{\tau}=-\frac{u K(I)}{\tau}=\Gamma_{12}^{2}=-\Gamma_{22}^{1} \\
\Gamma_{22}^{2}=\frac{G_{v}}{2 G}=\frac{\left(\frac{1}{\tau^{2}}\right)_{v}}{\frac{2}{\tau^{2}}}=-\frac{\tau_{v}}{\tau}=-\frac{v K(I)}{\tau}=\Gamma_{12}^{1}=-\Gamma_{11}^{2} .
\end{gathered}
$$

Agora segue da Proposição 3.2.2 que a equação de Gauss pode ser escrita como $K(I)=$ $K(I, I I)+\varepsilon\left(1-\|T\|^{2}\right)$. Assim, de acordo com a parametrização escolhida temos

$$
K(I)=\frac{e g-f^{2}}{\frac{1}{\tau^{4}}}+\varepsilon\left(1-\|T\|^{2}\right) .
$$


Portanto, $K(I)=\left(e g-f^{2}\right) \tau^{4}+\varepsilon\left(1-\|\nabla h\|^{2}\right)$. Segue de (3.18)

$$
\begin{aligned}
e_{v} & =\left\langle\left(\psi_{u u}\right)_{v}, N\right\rangle+\left\langle\psi_{u u}, N_{v}\right\rangle \\
f_{v} & =\left\langle\left(\psi_{u v}\right)_{v}, N\right\rangle+\left\langle\psi_{u v}, N_{v}\right\rangle \\
f_{u} & =\left\langle\left(\psi_{u v}\right)_{u}, N\right\rangle+\left\langle\psi_{u v}, N_{u}\right\rangle \\
g_{u} & =\left\langle\left(\psi_{v v}\right)_{u}, N\right\rangle+\left\langle\psi_{v v}, N_{u}\right\rangle .
\end{aligned}
$$

Como $N$ é unitário, temos que

$$
\begin{aligned}
e_{v}-f_{u}= & \left\langle\psi_{u u}, N_{v}\right\rangle-\left\langle\psi_{u v}, N_{u}\right\rangle \\
= & -\frac{u K(I)}{\tau}\left\langle\psi_{u}, N_{v}\right\rangle+\frac{v K(I)}{\tau}\left\langle\psi_{v}, N_{v}\right\rangle+c\left\langle N, N_{v}\right\rangle+\varepsilon\left(h_{u}^{2}-\frac{1}{\tau}\right)\left\langle\eta, N_{v}\right\rangle \\
& +\frac{v K(I)}{\tau}\left\langle\psi_{u}, N_{u}\right\rangle+\frac{u K(I)}{\tau}\left\langle\psi_{v}, N_{u}\right\rangle-f\left\langle N, N_{u}\right\rangle-\varepsilon h_{u} h_{v}\left\langle\eta, N_{u}\right\rangle \\
= & \frac{u K(I)}{\tau} f-\frac{v K(I)}{\tau} g+\varepsilon\left(h_{u}^{2}-\frac{1}{\tau^{2}}\right) \nu h_{v}-\frac{v K(I)}{\tau} e-\frac{u K(I)}{\tau} f-\varepsilon h_{u}^{2} h_{v} \nu \\
= & -\frac{v K(I)}{\tau}(g+e)-\frac{\varepsilon \nu h_{v}}{\tau^{2}} .
\end{aligned}
$$

De maneira análoga, temos

$$
f_{v}-g_{u}=\frac{u K(I)}{\tau}(e+g)-\frac{\varepsilon \nu h_{u}}{\tau^{2}} .
$$

Agora apresentaremos o teorema principal desta seção.

Teorema 3.4.1. Sejam $\Sigma$ uma superfície orientável e $\psi: \Sigma \longrightarrow \mathbb{M}^{2}(\varepsilon) \times \mathbb{R}$ uma imersão de curvatura Gaussiana constante. Se a forma quadrática A é uma métrica Riemanniana, então $(A, I I)$ é um par de Codazzi de curvatura extrinseca constante.

Demonstração. Sejam $(u, v)$ parâmetros isotérmicos sobre $\Sigma$ para $I$ e tomando $c=\frac{1}{\varepsilon K(I)-1}$ sabemos de (3.14) que

$$
A=\left(\frac{1}{\tau^{2}}+c h_{u}^{2}\right) d u^{2}+2\left(c h_{u} h_{v}\right) d u d v+\left(\frac{1}{\tau^{2}}+c h_{v}^{2}\right) d v^{2} .
$$

Fazendo

$$
\bar{E}=\frac{1}{\tau^{2}}+c h_{u}^{2}, \quad \bar{F}=c h_{u} h_{v} \quad e \quad \bar{G}=\frac{1}{\tau^{2}}+c h_{v}^{2}
$$

temos

$$
A=\bar{E} d u^{2}+2 \bar{F} d u d v+\bar{G} d v^{2}
$$


Por outro lado, a quarta coordenada das equações do Lema 3.4.4 é dada por

$$
\begin{aligned}
h_{u u} & =\left(-\frac{K(I) u}{\tau}\right) h_{u}+\left(-\frac{K(I) v}{\tau}\right) h_{v}+e \nu \\
h_{u v} & =\left(-\frac{K(I) v}{\tau}\right) h_{u}+\left(-\frac{K(I) u}{\tau}\right) h_{v}+f \nu \\
h_{v v} & =\left(\frac{K(I) u}{\tau}\right) h_{u}+\left(-\frac{K(I) v}{\tau}\right) h_{v}+g \nu .
\end{aligned}
$$

Então, usando (3.21), vamos calcular os símbolos de Christoffel associado à métrica Riemanniana $A$. Mas antes, vejamos que

$$
\begin{aligned}
& \bar{E}_{u}=\left(\frac{1}{\tau^{2}}+c h_{u}^{2}\right)_{u}=-\frac{2 d u}{\tau^{2}}+2 h_{u} h_{u u}=-\frac{2 u K(I)}{\tau^{3}}+2 c h_{u} h_{u u} \\
& \bar{E}_{v}=-\frac{2 v K(I)}{\tau^{3}}+2 c h_{u} h_{u v} \\
& \bar{F}_{u}=\left(c h_{u} h_{v}\right)_{u}=c\left(h_{u u} h_{v}+h_{u} h_{u v}\right) \\
& \bar{F}_{v}=c\left(h_{u v} h_{v}+h_{u} h_{v v}\right) \\
& \bar{G}_{u}=\left(\frac{1}{\tau^{2}}+c h_{v}^{2}\right)_{u}=-\frac{2 u K(I)}{\tau^{3}}+2 c h_{v} h_{v u} \\
& \bar{G}_{v}=-\frac{2 v K(I)}{\tau^{3}}+2 c h_{v} h_{v v}
\end{aligned}
$$

$\mathrm{e}$

$$
\overline{E G}-\bar{F}^{2}=\left(\frac{1}{\tau^{2}}+c h_{u}^{2}\right)\left(\frac{1}{\tau^{2}}+c h_{v}^{2}\right)-\left(c h_{u} h_{v}\right)^{2}=\frac{1}{\tau^{4}}\left(1+c\|\nabla h\|^{2}\right)
$$

Segue dos símbolos de Christoffel (1.2), aplicando (3.21) em (3.22) e usando (3.23) os símbolos de Christoffel associado à métrica $A$ são

$$
\begin{aligned}
\bar{\Gamma}_{11}^{1} & =-\frac{K(I) u}{\tau}+\frac{c \tau^{2} e \nu h_{u}}{1+c\|\nabla h\|^{2}} \\
\bar{\Gamma}_{11}^{2} & =\frac{K(I) v}{\tau}+\frac{c \tau^{2} e \nu h_{v}}{1+c\|\nabla h\|^{2}} \\
\bar{\Gamma}_{12}^{1} & =-\frac{K(I) v}{\tau}+\frac{c \tau^{2} f \nu h_{u}}{1+c\|\nabla h\|^{2}} \\
\bar{\Gamma}_{12}^{2} & =-\frac{K(I) u}{\tau}+\frac{c \tau^{2} f \nu h_{v}}{1+c\|\nabla h\|^{2}} \\
\bar{\Gamma}_{22}^{1} & =\frac{K(I) u}{\tau}+\frac{c \tau^{2} g \nu h_{u}}{1+c\|\nabla h\|^{2}} \\
\bar{\Gamma}_{22}^{2} & =-\frac{K(I) v}{\tau}+\frac{c \tau^{2} g \nu h_{v}}{1+c\|\nabla h\|^{2}} .
\end{aligned}
$$

Assim 


$$
\begin{aligned}
& e \Gamma_{12}^{2}+f\left(\Gamma_{12}^{2}-\Gamma_{11}^{1}\right)-g \Gamma_{12}^{2} \\
& =e\left(-\frac{v K(I)}{\tau}+\frac{f c \tau^{2} \nu h_{u}}{1-c\|\nabla\|^{2}}\right) \\
& +f\left(-\frac{K(I) u}{\tau}+\frac{c \tau^{2} f \nu h_{v}}{1+c\|\nabla h\|^{2}}+\frac{K(I) u}{\tau}-\frac{c \tau^{2} e \nu h_{u}}{1+c\|\nabla h\|^{2}}\right) \\
& -g\left(-\frac{K(I) u}{\tau}+\frac{c \tau^{2} f \nu h_{v}}{1+c \mid\|\nabla h\|^{2}}\right) \\
& =-\frac{v K(I)}{\tau}(e+g)-\left(\frac{c \tau^{2} \nu h_{v}}{1-c\|\nabla h\|^{2}}\right)\left(e g-f^{2}\right) \\
& e \Gamma_{22}^{1}+f\left(\Gamma_{22}^{2}-\Gamma_{12}^{1}\right)-g \Gamma_{12}^{2} \\
& =e\left(\frac{u K(I)}{\tau}+\frac{g c \tau^{2} \nu h_{u}}{1-c\|\nabla\|^{2}}\right) \\
& +f\left(-\frac{K(I) v}{\tau}+\frac{c \tau^{2} g \nu h_{v}}{1+c\|\nabla h\|^{2}}-\frac{K(I) v}{\tau}+\frac{c \tau^{2} f \nu h_{u}}{1+c\|\nabla h\|^{2}}\right) \\
& -g\left(-\frac{K(I) u}{\tau}+\frac{c \tau^{2} f \nu h_{v}}{1+c\|\nabla h\|^{2}}\right) \\
& =\frac{u K(I)}{\tau}(e+g)+\left(\frac{c \tau^{2} \nu h_{u}}{1-c|| \nabla h \|^{2}}\right)\left(e g-f^{2}\right) .
\end{aligned}
$$

Agora, da equação de Gauss (3.15) e $c=\frac{1}{\varepsilon K(I)-1}$ e $\nu^{2}=1-\|\nabla h\|^{2}$ temos

$$
\begin{aligned}
& e \Gamma_{12}^{2}+f\left(\Gamma_{12}^{2}-\Gamma_{11}^{1}\right)-g \Gamma_{12}^{2}= \\
& =-\frac{v K(I)}{\tau}(e+g)-\left(\frac{c \tau^{2} \nu h_{v}}{1-c\|\nabla h\|^{2}}\right)\left(\frac{K(I)-\varepsilon \nu^{2}}{\tau^{4}}\right) \\
& =-\frac{v K(I)}{\tau}(e+g)-\left(\frac{\nu h_{v}}{\tau^{4}}\right)\left(\frac{c K(I)-c \varepsilon \nu^{2}}{1-c\|\nabla h\|^{2}}\right) \\
& =-\frac{v K(I)}{\tau}(e+g)-\left(\frac{\nu h_{v} \varepsilon}{\tau^{2}}\right)\left(\frac{\varepsilon c K(I)-c \nu^{2}}{1-c\|\nabla h\|^{2}}\right) \\
& =-\frac{v K(I)}{\tau}(e+g)-\left(\frac{\nu h_{v} \varepsilon}{\tau^{2}}\right)\left(\frac{\varepsilon c K(I)-c-c\|\nabla h\|^{2}}{1-c\|\nabla h\|^{2}}\right) \\
& =-\frac{v K(I)}{\tau}(e+g)-\frac{\nu \varepsilon h_{v}}{\tau^{2}} .
\end{aligned}
$$

Prosseguindo analogamente

$$
e \Gamma_{22}^{1}+f\left(\Gamma_{22}^{2}-\Gamma_{12}^{1}\right)-g \Gamma_{12}^{2}=\frac{u K(I)}{\tau}(e+g)+\frac{\nu \varepsilon h_{u}}{\tau^{2}} .
$$


e das equações de Codazzi do Lema 3.4.4

$$
\begin{gathered}
e \Gamma_{12}^{2}+f\left(\Gamma_{12}^{2}-\Gamma_{11}^{1}\right)-g \Gamma_{12}^{2}=e_{v}-f_{u} \\
e \Gamma_{22}^{1}+f\left(\Gamma_{22}^{2}-\Gamma_{12}^{1}\right)-g \Gamma_{12}^{2}=f_{v}-g_{u} .
\end{gathered}
$$

Portanto o par $(A, I I)$ satisfaz às clássicas equações de Codazzi, isto é, $(A, I I)$ é um par de Codazzi. Agora para concluir que $K(A, I I)$ é constante basta aplicar o Lema 3.4.1, donde $K(I)$ é constante.

\subsection{Teorema Tipo Liebmann}

Para nosso próximo objetivo, precisamos do seguinte resultado o qual nos garante a existência de uma forma quadrática holomorfa associada ao par de Codazzi com curvatura extrínseca positiva constante.

Teorema 3.5.1 (Versão Abstrata de Liebmann). Dado $(I, I I) \in \mathcal{P}(\Sigma)$ tal que II $\in \mathcal{R}(\Sigma)$, então quaisquer duas das seguintes condiçôes implicam na terceira

(1) $(I, I I)$ é Codazzi;

(2) $K=K(I, I I)>0$ é constante;

(3) A (2,0)-parte de I é holomorfa para uma estrutura conforme dada por II, isto é, $p=$ $I\left(\partial_{z}, \partial_{z}\right)$ é uma função holomorfa para a estrutura conforme induzida por II.

Demonstração. Seja II uma métrica Riemanniana e consideramos $z$ um parâmetro local conforme para $I I$. Então podemos escrever

$$
\begin{gathered}
I=p d z^{2}+2 \lambda|d z|^{2}+\bar{p} d \bar{z}^{2} \\
I I=2 \rho|d z|^{2}
\end{gathered}
$$

e assim, o Tensor de Codazzi, $T_{S^{I I}}$, dada pela Proposição 1.6.3, satisfaz

$$
\left.\left\langle T_{S^{I I}}\left(\partial_{z}, \partial_{\bar{z}}\right), \partial_{z}\right)\right\rangle=2 \rho\left(\frac{K_{z}}{4 K}+\Gamma_{12}^{2}\right) .
$$

Portanto o resultado decorre das seguintes equivalências:

- $(I, I I)$ é Codazzi se, e somente se, $T_{S^{I I}}=0$;

- $K$ é constante se, e somente se, $K_{z}=K_{\bar{z}}=0$;

- $p=I\left(\partial_{z}, \partial_{z}\right)$ é holomorfa se, e somente se, $\Gamma_{12}^{1}=\Gamma_{12}^{2}=0$. 
A única afirmação não obvia é a última. Iremos checá-la. Desde que $z$ é um parâmetro conforme para $I I$ e $\mathrm{p}=I\left(\partial_{z}, \partial_{z}\right)$, então

$$
\begin{gathered}
p_{\bar{z}}=\partial_{\bar{z}} I\left(\partial_{z}, \partial_{z}\right)=2 I\left(\nabla_{\partial_{\bar{z}}} \partial_{z}, \partial_{z}\right)=2\left(p \Gamma_{12}^{1}+\lambda \Gamma_{12}^{2}\right) \\
\bar{p}_{z}=\partial_{z} I\left(\partial_{\bar{z}}, \partial_{\bar{z}}\right)=2 I\left(\nabla_{\partial_{z}} \partial_{\bar{z}}, \partial_{\bar{z}}\right)=2\left(\lambda \Gamma_{12}^{1}+p \Gamma_{12}^{2}\right)
\end{gathered}
$$

Como $p$ é holomorfa se, e somente se, $p_{\bar{z}}=\bar{p}_{z}=0$, então segue a afirmação.

Observe que para uma imersão $\psi: \Sigma \rightarrow \mathbb{M}^{2}(\varepsilon) \times \mathbb{R}$ com curvatura Gaussiana $K(I)>0$, se $\varepsilon=-1$ ou $K(I)>1$ se $\varepsilon=1$, sempre podemos considerar uma estrutura conforme induzida por II. De fato, pela equação de Gauss, temos que, se $\varepsilon=-1,0<K(I)=K-\nu^{2}$, e assim $K>0$. Analogamente, se $\varepsilon=1$, temos que $1<K(I)=K+\nu^{2}$, o que implica novamente que $K>0$, uma vez que $\nu^{2}<1$. Como a curvatura extrínseca é sempre positiva, $I I$ é definida e a menos de orientação, podemos considerar II positiva definida para obter uma estrutura conforme induzida por $I I$. Nesta estrutura, considere a $(2,0)$-parte de $A$, isto é

$$
Q d z^{2}=\left(\left\langle\psi_{z}, \psi_{z}\right\rangle+\frac{1}{\varepsilon K(I)-1} h_{z}^{2}\right) d z^{2}
$$

O próximo teorema caracteriza as imersões em que $Q d z^{2}$ é identicamente nula.

Teorema 3.5.2. [6] Seja $\Sigma \subset \mathbb{M}^{2} \times \mathbb{R}$ uma imersão de curvatura constante $K>0$ se $\varepsilon=-1$ (resp. $K>1$ se $\varepsilon=1$ ). Suponha que $Q d z^{2}$ é identicamente nula em $\Sigma$. Então $\Sigma$ é um pedaço de uma esfera rotacional invariante com curvatura Gaussiana constante K.

Demonstração. Dividiremos a demonstração em duas partes. Primeiro, mostraremos que a imersão deve ser helicoidal, isto é, $\psi$ é invariante sob um grupo contínuo de isometrias do espaço ambiente. Na segunda parte, demonstraremos que as órbitas desse movimento helicoidal são círculos sobre os slices $\mathbb{M}^{2} \times h_{0}$. Assim, concluiremos que $\psi$ é uma superfície de revolução.

Se $Q d z^{2} \equiv 0$, então existe uma função $\lambda$ tal que $I I=\lambda A$. Segue que a curvatura extrínseca do par $(A, I I)$ é dada por $K(A, I I)=\lambda^{2}$ e assim, pelo Lema 3.4.1, temos que $\lambda^{2}=K-\varepsilon$.

Seja $(u, v)$ coordenadas duplamente ortogonais para $(I, I I)$, lembrando que essas coordenadas estão disponíveis na vizinha de todo ponto não umbílico bem como sobre o interior do conjunto dos pontos umbílicos. Então, considerando esses pontos e, usando que esse conjunto é denso em $\Sigma$, temos que as propriedades obtidas podem ser estendidas para toda superfície por continuidade.

Desde que $I I=\sqrt{K(I)-\varepsilon} A$ temos

$$
\begin{aligned}
k_{1} E & =\sqrt{K(I)-\varepsilon}\left(E+\frac{\varepsilon}{K(I)-\varepsilon} h_{u}^{2}\right) \\
0 & =h_{u} h_{v} \\
k_{2} G & =\sqrt{K(I)-\varepsilon}\left(G+\frac{\varepsilon}{K(I)-\varepsilon} h_{v}^{2}\right) .
\end{aligned}
$$

De (3.26) é fácil ver que o conjunto dado pela união do interior do conjunto, onde $h_{u}=0$ 
e a do interior do conjunto onde $h_{v}=0$ é denso. De fato, se $h_{v}(p) \neq 0$ para algum ponto $p \in \Sigma$, existe uma vizinha $V$ de $p$ onde $h_{v} \neq 0$. Mas como $h_{v} h_{u}=0$, segue que $h_{u}=0$ em $V$. Consequentemente podemos assumir que $h_{u}=0$ na vizinhança onde $(u, v)$ são tomados.

Então de (3.25), a curvatura principal $k_{1}=\sqrt{K(I)-\varepsilon}$ é uma constante positiva. Além disso, de (3.6), tem que $\nu_{u}=d \nu\left(\partial_{u}\right)=-\left\langle k_{1} \partial_{u}, T\right\rangle=-k_{1} h_{u}=0$. Logo, da equação de Gauss, $\left(k_{2}\right)_{u}=0$.

Agora, (3.27) pode ser reescrita como

$$
k_{1}\left(k_{2}-k_{1}\right) \bar{G}=\varepsilon h_{v}^{2}
$$

Uma vez que estamos considerando uma vizinhança sem pontos umbílicos, observamos que se $k_{1} \equiv k_{2}$ então de (3.28), $h_{v}$ também é identicamente nula. Assim, teríamos $h$ constante, isto é, a superfície estaria sobre um Slice e então $K(I)=\varepsilon$, o que é uma contradição. Logo, assumimos $k_{1} \neq k_{2}$ em nossa vizinhança e $\bar{G}_{u}=0$ de (3.28).

Se consideramos a equação de Codazzi (3.4) para $X=\partial_{u}$ e $Y=\partial_{v}$, obtemos

$$
\left(k_{2}-k_{1}\right) \nabla_{\partial_{u}} \partial_{v}=\nabla_{\partial_{u}} k_{2} \partial_{v}-\nabla_{\partial_{v}} k_{1} \partial_{u}=\varepsilon \nu\left(\left\langle\partial_{v}, T\right\rangle \partial_{u}-\left\langle\partial_{u}, T\right\rangle \partial_{v}\right)=\varepsilon \nu h_{v} \partial_{u}
$$

Logo, $\nabla_{\partial_{u}} \partial_{v}=\frac{E_{v}}{2 E} \partial_{u}$, então $(\log E)_{u v}=\left(\frac{E_{v}}{E}\right)_{u}=0$. Isto é, a função $E(u, v)$ pode ser escrita como $E(u, v)=E_{1}(u) E_{2}(v)$ para funções positivas $E_{1}$ e $E_{2}$.

Agora tomando novos parâmetros $(x, y)$ tais que $d x=\sqrt{E_{1}(u)} d u$ e $y=v$. Então, a primeira e segunda forma fundamental, $h$ e $\nu$ apenas dependem de $y$, isto é, as funções $E, G, k_{1}, k_{2}, h$ e $\nu$ não depende de $(x, y)$, mas apenas de $y$.

Assim, as imersões $\psi(x, y)$ e $\bar{\psi}(x, y)=\psi\left(x+x_{0}, y\right)$ para um adequado $x_{0}$, têm as mesmas funções $E, G, k_{1}, k_{2}, h$ e $\nu$. Então $\psi$ e $\bar{\psi}$ somente diferem por uma isometria do espaço ambiente para cada $x_{0}$ (ver [12]), isto é, $\psi$ é helicoidal e as órbitas são dadas por $\beta(t)=\psi(x+t, y)$.

Na segunda parte da prova, mostraremos que $\psi$ é uma superfície de rotação. Observe que $\beta(t)$ está contida em um plano horizontal devido a função altura depender apenas de $y$.

Em particular, $\beta(t) \subset \mathbb{M}^{2}(\varepsilon) \times\{y\} \equiv \mathbb{M}^{2}(\varepsilon)$ é invariante sob um grupo de isometrias de $\mathbb{M}^{2}(\varepsilon)$. Então, a curvatura de $\beta$ em $\mathbb{M}^{2}(\varepsilon)$ é constante.

Portanto se $\varepsilon=1$ então $\beta$ esta contida sobre um círculo de $\mathbb{S}^{2}$. Por outro lado, se $\varepsilon=-1$, $\beta$ esta contida em um círculo de $\mathbb{H}^{2}$ se, e somente se, sua curvatura é maior que 1 . Os detalhes dessa afirmação podem ser visto em [28].

Para o caso em que a curva está contida em $\mathbb{H}^{2}$, um cálculo fácil nos dá $\nabla_{\partial_{x}} \partial_{x}=-\frac{1}{2} \frac{E_{y}}{G} \partial_{y}+$ $k_{1} E N$, onde $\nabla$ denota a conexão de Levi-Civita em $\mathbb{H}^{2} \times \mathbb{R}$. Além disso, $\beta$ pode ser parametrizada pelo comprimento de arco como $\beta(s)=\psi\left(x+\frac{s}{\sqrt{E(y)}}, y\right)$. Então, o quadrado de sua curvatura é dada por

$$
\left|\nabla_{\beta^{\prime}(s)} \beta^{\prime}(s)\right|^{2}=\frac{1}{4 E(y)^{2}}\left(\frac{E_{y}(y)^{2}}{E(y)}+k_{1}^{2} E(y)^{2}\right) \geq k_{1}^{2}=K(I)+1>1 .
$$


Então, $\beta$ está contida em um círculo em qualquer caso e $\psi$ deve ser uma superfície de rotação. Finalmente, a prova finaliza como consequência do próximo lema.

Lema 3.5.1. Seja $\psi: \Sigma \longrightarrow \mathbb{M}^{2}(\varepsilon) \times \mathbb{R}$ uma superfície de rotação que a curvatura principal $k_{1}$ associada com seus paralelos coincidem. Se $k_{1}>1$ para $\varepsilon=-1$ ou $k_{1}>0$ para $\varepsilon=1$ então, a menos de isometria, $\Sigma$ é um pedaço de uma esfera rotacionalmente invariante com curvatura Gaussiana $K$ constante.

Demonstração. Ver [6].

Teorema 3.5.3 (do tipo Liebmann). Dada uma constante real $K(I)$, existe, a menos de isometria, uma única superfície completa de curvatura Gaussiana constante $K(I)>1$ em $\mathbb{S}^{2} \times \mathbb{R} e$ uma única de curvatura Gaussiana constante $K(I)>0$ em $\mathbb{H}^{2} \times \mathbb{R}$. Além disso, estas superfícies são rotacionalmente simétricas.

Demonstração. A parte da existência do resultado foi apresentado na Seção 3.3. Então, precisamos apenas mostrar a unicidade.

Sejam $\Sigma$ uma superfície e $\psi: \Sigma \longrightarrow \mathbb{M}^{2}(\varepsilon) \times \mathbb{R}$ uma imersão completa com curvatura Gaussiana constante $K(I)$ positiva. Desde que $K(I)$ é positiva, então pelo Teorema de Bonnet ${ }^{\dagger}$, $\Sigma$ é compacta. Além disso, considerando o recobrimento universal se necessário podemos assumir $\Sigma$ simplesmente conexa. Note que toda superfície compacta imersa em $\mathbb{M}^{2}(\varepsilon) \times \mathbb{R}$ é orientável, então $\Sigma$ é uma esfera topológica pelo teorema de Gauss-Bonnet ${ }^{\ddagger}$.

Por outro lado, pelo Teorema 3.4.1, $(A, I I)$ é um par de Codazzi com curvatura extrínseca constante e positiva, de onde obtemos, pelo Teorema 3.5.1 que a parte $(2,0)$ de $A$ é holomorfa para a estrutura induzida por $I I$, isto é, se $z$ é um parâmetro conforme para $I I$, então

$$
Q d z^{2}=A\left(\partial_{z}, \partial_{z}\right)
$$

é uma diferencial quadrática holomorfa em $\Sigma$. Portanto, como $\Sigma$ é um esfera topológica, pelo Teorema 1.6.4, $Q d z^{2}$ deve ser identicamente zero sobre $\Sigma$. Então, segue do Teorema 3.5.2, o nosso resultado.

\footnotetext{
${ }^{\dagger}$ Ver [8], cap. 5, seção 5.4

${ }^{\ddagger}$ Ver [8], cap. 4, seção 4.5
} 


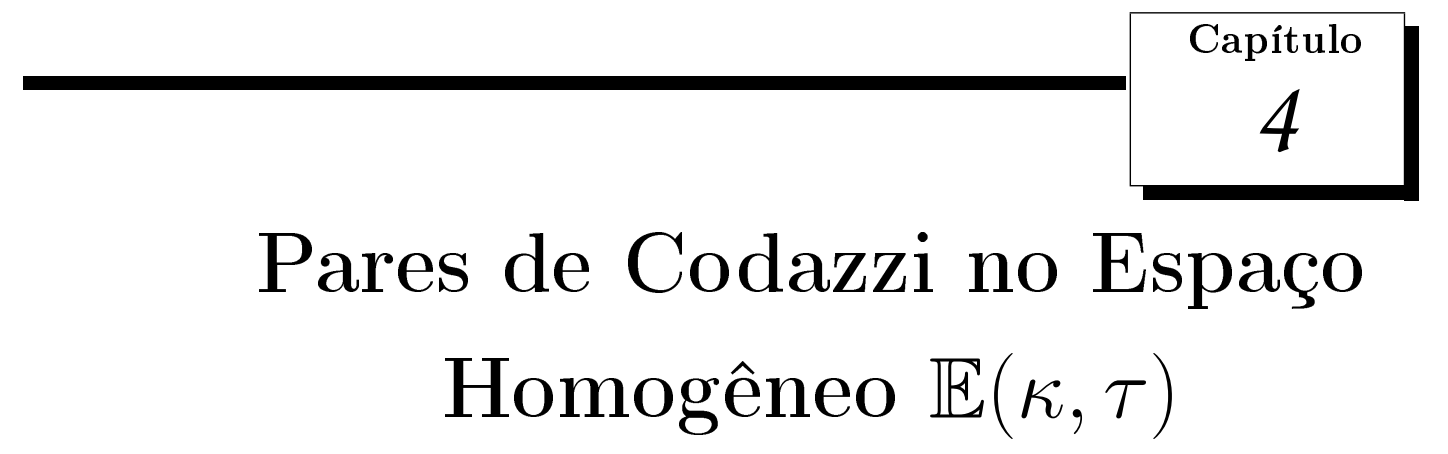

Sabe-se da teoria de superfícies que existe uma diferencial quadrática holomorfa definida em qualquer superfície de curvatura média constante, em resumo, $H$-superfície, imersa no espaço homogêneo $\mathbb{E}(\kappa, \tau)$, dada por U.Abresch e H.Rosenberg, (ver [7]), chamada à diferencial de Abresch-Rosenberg. Porém, não existia par de Codazzi sobre tais $H$-superfícies associadas à diferencial de Abresch-Rosenberg quando $\tau \neq 0$. Inspirado nos estudos de José Espinar e Haimer Trejos em [15], o objetivo deste capítulo é encontrar par geométrico Codazzi definido sobre qualquer $H$-superfície em $\mathbb{E}(\kappa, \tau)$, quando $\tau \neq 0$, cujo $(2,0)$-parte é a diferencial AbreschRosenberg.

\subsection{Introdução}

Neste capítulo trataremos de superfícies de curvatura média constante em espaços homogêneos. Nos últimos anos, o tema das $H$-superfícies em espaços homogêneos cresceu rapidamente motivado, principalmente, pelo resultado de U. Abresch e H. Rosenberg em [1] e [2], sobre a classificação de esferas topológicas com curvatura média constante.

Tais autores provaram que para superfícies com curvatura média constante imersas nos espaços homogêneos $\mathbb{E}(\kappa, \tau)$, existe uma diferencial quadrática holomorfa e denominada diferencial Abresch-Rosenberg. Seguem daí as classificações de superfícies cuja diferencial quadrática é nula e assim temos a generalização do Teorema de Hopf. Motivado pela existência dessa diferencial quadrática, veremos os pares de Codazzi sobre qualquer $H$-superfícies em $\mathbb{E}(\kappa, \tau)$. Além disso, obteremos resultados de classificação, no qual classificaremos superfícies completas de curvatura média constante com funções Abresch-Rosenberg constante não nula. 


\subsection{Resultados Básicos}

\subsubsection{Variedades Riemannianas Homogêneas $\mathbb{E}(\kappa, \tau)$}

Uma variedade homogênea simplesmente conexa $\mathbb{E}(\kappa, \tau)$ é uma submersão Riemanniana $\pi: \mathbb{E}(\kappa, \tau) \longrightarrow \mathbb{M}^{2}(\kappa)$ sobre uma superfície simplesmente conexa de curvatura $\kappa$ constante. As fibras, isto é, as imagens inversas de um ponto em $\mathbb{M}^{2}(\kappa)$ por $\pi$, são trajetórias de um campo de vetores de Killing unitário $\xi$, chamada campo de vetores verticais.

Denote por $\bar{\nabla}, \wedge, \bar{R}$ a conexão, produto vetorial na métrica de $\mathbb{E}(\kappa, \tau)$ e o Tensor curvatura de $\mathbb{E}(\kappa, \tau)$, respectivamente. Então seguem os seguintes resultados, cujas demonstrações se encontram em [13].

Proposição 4.2.1. Seja $\mathbb{E}(\kappa, \tau)$ como dito acima. Então existe uma função $\tau: M \longrightarrow \mathbb{R}$ tal que

$$
\bar{\nabla}_{X} \xi=\tau X \wedge \xi
$$

onde $\tau$ é a curvatura do fibrado. Note que $\tau=0$ implica que $\mathbb{E}(\kappa, \tau)$ é o espaço produto.

Proposição 4.2.2. Seja $\pi: \mathbb{E}(\kappa, \tau) \longrightarrow \mathbb{M}^{2}(\kappa)$ uma submersão Riemanniana com o campo de killing unitário $\xi$. Seja $\left\{E_{1}, E_{2}\right\} \in T \mathbb{E}$ uma base ortonormal de vetores horizontais tal que $\left\{E_{1}, E_{2}, \xi\right\}$ é orientada positivamente. Então

$$
\left\langle\bar{R}\left(E_{1}, E_{2}\right) E_{1}, E_{2}\right\rangle=\kappa-3 \tau^{2} \quad e \quad\left\langle\bar{R}\left(E_{i}, \xi\right) E_{i}, \xi\right\rangle=\tau^{2}
$$

onde $\bar{K}$ é a curvatura seccional de qualquer plano gerado por $E_{1}, E_{2} \in \mathfrak{X}(\mathbb{E}(\kappa, \tau))$

Utilizaremos o resultado acima, para expressar o Tensor curvatura do espaço $\mathbb{E}(\kappa, \tau)$.

Teorema 4.2.1. Seja $\mathbb{E}(\kappa, \tau)$ um espaço homogêneo com campo de Killing unitário $\xi$. Para todos campos vetoriais $X, Y, Z$ e $W \in \mathfrak{X}(\mathbb{E}(\kappa, \tau))$ temos:

$$
\langle\bar{R}(X, Y) Z, W\rangle=\left(\kappa-3 \tau^{2}\right)\left\langle R_{0}(X, Y) Z, W\right\rangle+\left(\kappa-4 \tau^{2}\right)\left\langle R_{1}(\xi, X, Y) Z, W\right\rangle,
$$

com $R_{0}(X, Y) Z=\langle X, Z\rangle Y-\langle Y, Z\rangle Y$ e

$R_{1}(V, X, Y) Z=\langle Y, V\rangle\langle Z, V\rangle X+\langle Y, Z\rangle\langle X, V\rangle V-\langle X, Z\rangle\langle Y, V\rangle V-\langle X, V\rangle\langle Z, V\rangle Y$.

Demonstração. Inicialmente, vamos decompor os campos $X, Y, Z$ e $W$ em sua parte horizontal e vertical, com respeito a $\xi$, isto é, $X=\bar{X}+\xi x, Y=\bar{Y}+\xi y, Z=\bar{Z}+\xi z$ e $W=\bar{W}+\xi w$, onde $x=\langle X, \xi\rangle, y=\langle Y, \xi\rangle, z=\langle Z, \xi\rangle$ e $w=\langle W, \xi\rangle$. Portanto, pela multilinearidade do tensor 
curvatura, temos

$$
\begin{aligned}
& \langle\bar{R}(X, Y) Z, W\rangle=\langle\bar{R}(\bar{X}+\xi x, \bar{Y}+\xi y)(\bar{Z}+\xi z), \bar{W}+\xi w\rangle= \\
= & \langle\bar{R}(\bar{X}, \bar{Y}) \bar{Z}, \bar{W}\rangle+\langle\bar{R}(\xi x, \bar{Y}) \bar{Z}, \bar{W}\rangle+\langle\bar{R}(\bar{X}, \xi y) \bar{Z}, \bar{W}\rangle \\
+ & \langle\bar{R}(\xi x, \xi y) \bar{Z}, \bar{W}\rangle+\langle\bar{R}(\bar{X}, \bar{Y}) \xi z, \bar{W}\rangle+\langle\bar{R}(\xi x, \bar{Y}) \xi z, \bar{W}\rangle \\
+ & \langle\bar{R}(\bar{X}, \xi y) \xi z, \bar{W}\rangle+\langle\bar{R}(\xi x, \xi y) \xi z, \bar{W}\rangle+\langle\bar{R}(\bar{X}, \bar{Y}) \bar{Z}, \xi w\rangle \\
+ & \langle\bar{R}(\xi x, \bar{Y}) \bar{Z}, \xi w\rangle+\langle\bar{R}(\bar{X}, \xi y) \bar{Z}, \xi w\rangle+\langle\bar{R}(\xi x, \xi y) \bar{Z}, \xi w\rangle \\
+ & \langle\bar{R}(\bar{X}, \bar{Y}) \xi z, \xi w\rangle+\langle\bar{R}(\xi x, \bar{Y}) \xi z, \xi w\rangle+\langle\bar{R}(\bar{X}, \xi y) \xi z, \xi w\rangle \\
+ & \langle\bar{R}(\xi x, \xi y) \xi z, \xi w\rangle .
\end{aligned}
$$

Devido às propriedades anti-simétrica do tensor curvatura, temos

$$
x y\langle\bar{R}(\xi, \xi) A, B\rangle=-x y\langle\bar{R}(\xi, \xi) A, B\rangle,
$$

que implica $\langle\bar{R}(\xi, \xi) A, B\rangle=0$, e modo análogo, $\langle\bar{R}(A, B) \xi, \xi\rangle=0$. O termo onde $\xi$ aparece apenas uma vez são nulos, pois existe uma base ortonormal em que a matriz de $\bar{R}$ é diagonal. Então, temos

$$
\begin{aligned}
\langle\bar{R}(X, Y) Z, W\rangle=\langle\bar{R}(\bar{X}, \bar{Y}) \bar{Z}, \bar{W}\rangle & +y w\langle\bar{R}(\bar{X}, \xi) \bar{Z}, \xi\rangle+y z\langle\bar{R}(\bar{X}, \xi) \xi, \bar{W}\rangle \\
& +x w\langle\bar{R}(\xi, \bar{Y}) \bar{Z}, \xi\rangle+x z\langle\bar{R}(\xi, \bar{Y}) \xi, \bar{W}\rangle .
\end{aligned}
$$

Seja $E_{1}, E_{2}$ base ortonormal dos vetores horizontais como na Proposição 4.2.2. Então

$$
\left\langle\bar{R}\left(E_{1}, E_{2}\right) E_{1}, E_{2}\right\rangle=\kappa-3 \tau^{2} \quad \text { e }\left\langle\bar{R}\left(E_{i}, \xi\right) E_{i}, \xi\right\rangle=\tau^{2}
$$

A partir disso, se escrevermos

$$
\bar{X}=x_{1} E_{1}+x_{2} E_{2}, \quad \bar{Y}=y_{1} E_{1}+y_{2} E_{2} \text { e } \bar{Z}=z_{1} E_{1}+z_{2} E_{2}
$$

temos que

$$
\left.\langle\bar{R}(\bar{X}, \bar{Y}) \bar{Z}, \bar{W}\rangle=\left(\kappa-3 \tau^{2}\right)[\langle\bar{X}, \bar{Z}\rangle\langle\bar{Y}, \bar{W}\rangle)-\langle\bar{Y}, \bar{Z}\rangle\langle\bar{X}, \bar{W}\rangle\right]
$$

Usando agora

$$
\begin{aligned}
\left\langle\bar{R}\left(E_{1}, E_{3}\right) E_{1}, E_{3}\right\rangle & =\left\langle\bar{R}\left(E_{2}, E_{3}\right) E_{2}, E_{3}\right\rangle=\tau^{2} \quad e \\
\left.\bar{R}\left(E_{1}, E_{3}\right) E_{2}, E_{3}\right\rangle & =0
\end{aligned}
$$

temos

$$
\langle\bar{R}(\bar{X}, \xi) \bar{Z}, \xi\rangle=\langle\bar{X}, \bar{Z}\rangle \tau^{2}
$$


Analogamente,

$$
\begin{aligned}
\langle\bar{R}(\bar{X}, \xi) \xi, \bar{W}\rangle & =-\langle\bar{X}, \bar{W}\rangle \tau^{2} \\
\langle\bar{R}(\xi, \bar{Y}) \bar{Y}, \xi\rangle & =-\langle\bar{Y}, \bar{Z}\rangle \tau^{2} \\
\langle\bar{R}(\xi, \bar{Y}) \xi, \bar{W}\rangle & =\langle\bar{Y}, \bar{W}\rangle \tau^{2}
\end{aligned}
$$

Portanto

$$
\begin{aligned}
& \langle\bar{R}(X, Y) Z, W\rangle= \\
& \left.=\left(\kappa-3 \tau^{2}\right)[\langle\bar{X}, \bar{Z}\rangle\langle\bar{Y}, \bar{W}\rangle)-\langle\bar{Y}, \bar{Z}\rangle\langle\bar{X}, \bar{W}\rangle\right] \\
& +\tau^{2}[y w\langle\bar{X}, \bar{Z}\rangle-y z\langle\bar{X}, \bar{W}\rangle-x w\langle\bar{Y}, \bar{Z}\rangle+x z\langle\bar{Y}, \bar{W}\rangle] \\
& =\left(\kappa-3 \tau^{2}\right)[(\langle X, Z\rangle\langle Y, W\rangle)-\langle Y, Z\rangle\langle X, W\rangle] \\
& -\left(\kappa-4 \tau^{2}\right)[\langle X, Z\rangle\langle Y, \xi\rangle\langle W, \xi\rangle+\langle Y, W\rangle\langle X, \xi\rangle\langle Z, \xi\rangle] \\
& -\left(\kappa-4 \tau^{2}\right)[-\langle X, W\rangle\langle Y, \xi\rangle\langle Z, \xi\rangle-\langle Y, Z\rangle\langle X, \xi\rangle\langle W, \xi\rangle] \\
& =\left(\kappa-3 \tau^{2}\right)\left\langle R_{0}(X, Y) Z, W\right\rangle+\left(\kappa-4 \tau^{2}\right)\left\langle R_{1}(\xi, X, Y) Z, W\right\rangle .
\end{aligned}
$$

\subsubsection{Superfícies Imersas em $\mathbb{E}(\kappa, \tau)$}

Seja $\Sigma \subset \mathbb{R}(\kappa, \tau)$ uma superfície orientada conexa imersa. Dotaremos $\Sigma$ com a métrica induzida de $\mathbb{E}(\kappa, \tau)$, chamada a primeira forma fundamental, na qual denotaremos por $\langle$,$\rangle . Sejam$ $\nabla$ e $R$ a conexão de Levi-Civita e o tensor curvatura de $\Sigma$, respectivamente. Denotaremos por $A$ o operador forma, isto é, $A X=-\nabla_{X} N, \forall X \in \mathfrak{X}(\Sigma)$, onde $N$ é o campo normal unitário ao longo de $\Sigma$. Então $I I(X, Y)=\langle A X, Y\rangle$ é a segunda forma fundamental de $\Sigma$. Se $\Sigma$ é orientável, podemos definir uma estrutura complexa $J$ sobre $\Sigma$, dada por $J\left(e_{1}\right)=e_{2}$ e $J\left(e_{2}\right)=-e_{1}$, onde $\left\{e_{1}, e_{2}\right\} \subset T_{p} \Sigma$ é uma base ortonormal positiva. Geometricamente, $J$ representa uma rotação de $\frac{\pi}{2}$ em $T_{p} \Sigma$, no sentido anti-horário, tal que $J X=N \wedge X, \forall X \in \mathfrak{X}(\Sigma)$.

Se $\nu=\langle N, \xi\rangle$ e $T=\xi-\nu N$, então $\nu$ é o componente normal do campo vertical $\xi$, chamado função ângulo, e $T$ é um campo em $\mathfrak{X}(\Sigma)$ chamado componente tangente do campo vertical $\xi$.

Lembrando que no espaço produto $\mathbb{M}^{2}(\kappa) \times \mathbb{R}$, temos uma projeção natural sobre a fibra $\sigma: \Sigma \longrightarrow \mathbb{R}$, onde podemos definir a restrição de $\sigma$ para a superfície, isto é, $h=\left.\sigma\right|_{\Sigma}: \Sigma \longrightarrow \mathbb{R}$. $A$ função $h$ é chamada função altura de $\Sigma$. Então, em $\mathbb{M}^{2}(\kappa) \times \mathbb{R}$, podemos observar que $\bar{\nabla} \sigma=\xi$ e assim, $T$ é a projeção de $\bar{\nabla} \sigma$ sobre o plano tangente, $\nabla h=T$.

Apresentaremos agora, a prova de que as equações de compatibilidade são condições necessárias para existência da Imersão. 
Lema 4.2.1. Sejam $\Sigma \subset \mathbb{E}(\kappa, \tau)$ uma superfície imersa com campo unitário normal $N$ e operador forma A. Sejam $T$ e $\nu$ o componente tangente do campo vertical e a função ângulo respectivamente. Então, dados $X, Y \in \mathfrak{X}(\Sigma)$, valem as seguintes equações:

$$
\begin{array}{rlr}
K & =K_{e}+\tau^{2}+\left(\kappa-4 \tau^{2}\right) \nu^{2} & \text { Gauss } \\
T_{S}(X, Y) & =\left(\kappa-4 \tau^{2}\right) \nu(\langle Y, T\rangle X-\langle X, T\rangle Y) & \text { Codazzi } \\
\nabla_{X} T & =\nu(A X-\tau J X) & \\
d \nu(X) & =\nu\langle\tau J X-A X, T\rangle & \\
1 & =\|T\|^{2}+\nu^{2},
\end{array}
$$

onde $K$ denota a curvatura Gaussiana de $\Sigma, K_{e}=\operatorname{det} A$ a curvatura extrínseca e $T_{S}$ é o tensor dado por

$$
T_{S}(X, Y)=\nabla_{X} A Y-\nabla_{Y} A X-A([X, Y]) \quad X, Y \in \mathfrak{X}(\Sigma) .
$$

Demonstração. Primeiro, decompondo o campo vertical $\xi$ na sua parte tangente e normal, isto é, $\xi=T+\nu N$, onde $\mathrm{T} \in \mathfrak{X}(\Sigma)$. Desde que $\xi$ é um vetor unitário temos

$$
1=\langle\xi, \xi\rangle+\nu^{2}=\|T\|^{2}+\nu^{2} .
$$

Pela fórmula de Gauss

$$
\nabla_{X} Y=\bar{\nabla}_{X} Y-\langle A X, Y\rangle N
$$

$\forall X, Y \in \mathfrak{X}(\Sigma)$ e usando a equação (4.1), temos

$$
\begin{aligned}
\tau X \wedge \xi=\bar{\nabla}_{X} \xi & =\bar{\nabla}_{X}(T+\nu N)=\bar{\nabla}_{X} T+d \nu(X) N+\nu \bar{\nabla}_{X} N \\
& =\nabla_{X} T+\langle A X, T\rangle N+d \nu(X) N+\nu \bar{\nabla}_{X} N \\
& =\nabla_{X} T+\langle A X, T\rangle N+d \nu(X) N-\nu A X \\
& =\left(\nabla_{X} T-\nu A X\right)+(\langle A X, T\rangle+d \nu(X)) N
\end{aligned}
$$

Por outro lado,

$$
\begin{aligned}
\tau X \wedge \xi=\tau X \wedge(T+\nu N) & =\tau X \wedge T+\tau \nu X \wedge N \\
= & \tau(\langle J X, T\rangle N-\nu J X)
\end{aligned}
$$

Assim,

$$
\left(\nabla_{X} T-\nu A X\right)+(\langle A X, T\rangle+d \nu(X)) N=\tau(\langle J X, T\rangle N-\nu J X),
$$

então, obtemos (4.5) e (4.6) igualando as partes tangente e normal da expressão acima. 
Agora dados $X, Y, Z \in \mathfrak{X}(\Sigma)$, as equações de Gauss e Codazzi de uma superfície são dada respectivamente, por:

$$
\begin{aligned}
\langle R(X, Y) Z, W\rangle-\bar{R}(X, Y) Z, W\rangle & =\langle A X, Z\rangle\langle A Y, W\rangle-\langle A X, W\rangle\langle A Y, Z\rangle \\
\bar{R}(X, Y) N & =\nabla_{X} A Y-\nabla_{Y} A X-A[X, Y]
\end{aligned}
$$

Segue de (4.2) que

$$
\begin{aligned}
& \bar{R}(X, Y) N= \\
& =\left(\kappa-3 \tau^{2}\right) R_{0}(X, Y) N+\left(\kappa-4 \tau^{2}\right) R_{1}(\xi, X, Y) N \\
& =\left(\kappa-3 \tau^{2}\right)[\langle X, N\rangle Y-\langle Y, N\rangle X] \\
& +\left(\kappa-4 \tau^{2}\right)[\langle Y, \xi\rangle\langle N, \xi\rangle X+\langle Y, N\rangle\langle X, \xi\rangle \xi] \\
& +\left(\kappa-4 \tau^{2}\right)[-\langle X, \xi\rangle\langle Y, \xi\rangle \xi-\langle X, \xi\rangle\langle N, \xi\rangle Y] \\
& =\left(\kappa-4 \tau^{2}\right)[\langle Y, T+\nu N\rangle\langle N, T+\nu N\rangle X-\langle X, T+\nu N\rangle\langle N, T+\nu N\rangle Y] \\
& =\left(\kappa-4 \tau^{2}\right) \nu[\langle Y, T+\nu N\rangle X-\langle X, T+\nu N\rangle Y] \\
& =\left(\kappa-4 \tau^{2}\right) \nu[\langle Y, T\rangle X-\langle X, T\rangle Y]
\end{aligned}
$$

Assim, a equação de Codazzi pode ser reescrita como

$$
\begin{aligned}
T_{S}(X, Y) & =\bar{R}(X, Y) N=\nabla_{X} A Y-\nabla_{Y} A X-A[X, Y] \\
& =\left(\kappa-4 \tau^{2}\right) \nu[\langle Y, T\rangle X-\langle X, T\rangle Y]
\end{aligned}
$$

Concluímos (4.4).

Utilizando a Proposição 4.2.1, podemos substituir $\langle\bar{R}(X, Y) Z, W\rangle$ e reescrever a equação de Gauss como

$$
\begin{aligned}
& \langle R(X, Y) Z, W\rangle-\left(\kappa-3 \tau^{2}\right)\left\langle R_{0}(X, Y) Z, W\right\rangle-\left(\kappa-4 \tau^{2}\right)\left\langle R_{1}(T, X, Y) Z, W\right\rangle \\
& =\langle A X, Z\rangle\langle A X, W\rangle-\langle A X, W\rangle\langle A Y, Z\rangle .
\end{aligned}
$$

Ainda podemos considerar $X$ e $Y$ ortonormais, e escolher $X=Z$ e $W=Y$. Daí,

$$
\begin{aligned}
R_{0}(X, Y) X & =\langle X, X\rangle Y-\langle Y, X\rangle X=\|X\|^{2} Y=Y \\
R_{1}(T, X, Y) X & =\langle Y, T\rangle\langle X, T\rangle X-\langle Y, T\rangle T-\langle X, T\rangle\langle X, T\rangle Y
\end{aligned}
$$

Assim, fazendo o produto interno dos primeiros e segundos membros das igualdades acima com $Y$, obtemos

$$
\begin{aligned}
\left\langle R_{0}(X, Y) X, Y\right\rangle & =1 \\
\left\langle R_{1}(T, X, Y) X, Y\right\rangle & =-\langle Y, T\rangle^{2}-\langle X, T\rangle^{2}=-\|T\|^{2}=-\left(1-\nu^{2}\right) .
\end{aligned}
$$


Além disso,

$$
\langle A X, X\rangle\langle A Y, Y\rangle-\langle A X, Y\rangle\langle A Y, X\rangle=K_{e} .
$$

Logo, (4.9) pode ser reescrita como

$$
\langle R(X, Y) Z, W\rangle-\left[\left(\kappa-3 \tau^{2}\right)+\left(\kappa-4 \tau^{2}\right)\left(\nu^{2}-1\right)\right]=K_{e}
$$

Portanto,

$$
K=K_{e}+\kappa-3 \tau^{2}-\left(\kappa-4 \tau^{2}\right)\left(1-\nu^{2}\right)=K_{e}+\tau^{2}+\left(\kappa-4 \tau^{2}\right) \nu^{2}
$$

O próximo resultado foi obtido por Benoit Daniel (Ver [13]), e afirma que, para existir a imersão de uma superfície $\Sigma$ num espaço homogêneo tridimensional $\mathbb{E}(\kappa, \tau)$, é necessário e suficiente que $\Sigma$ satisfaça às equações de compatibilidade dadas no Lema 4.2.1

Teorema 4.2.2 (Benoit Daniel). Seja $\Sigma$ uma superfície Riemanniana simplesmente conexa orientada.Sejam $\langle$,$\rangle sua métrica e \nabla$ sua conexão de Levi-Civita. Sejam A um campo de operadores simétricos $A_{p}: T_{p} \Sigma \longrightarrow T_{p} \Sigma, T$ um campo de vetores em $\Sigma$ e $\nu$ uma função suave tal que $1=\|T\|^{2}+\nu^{2}$.

Seja $\xi$ um campo vertical em $\mathbb{E}(\kappa, \tau)$, onde $\kappa$ é a curvatura da base da fibração e $\tau$ a curvatura do fibrado. Então, existe uma imersão isométrica $f: \Sigma \longrightarrow \mathbb{E}(\kappa, \tau)$ tal que o operador com respeito ao normal $N$ associado a $f$ é $d f \circ S \circ d f^{-1}$ e tal que $\xi=d f(T)+\nu N$ se, e somente se, $\left(d s^{2}, A, T, \nu\right)$ satisfaz às equaçôes de compatibilidade. Neste caso, a imersão é única a menos de isometrias globais de $\mathbb{E}(\kappa, \tau)$ preservando a orientação da fibra e da base da fibração.

\subsubsection{H-Superfícies Imersas em $\mathbb{E}(\kappa, \tau)$ e a diferencial de Abresch-Rosenberg}

Agora veremos as equações fundamentais para uma imersão $\psi: \Sigma \longrightarrow \mathbb{E}(\kappa, \tau)$ em termos de um parâmetro conforme $z=u+i v$. Identificaremos $\psi(\Sigma)$ com $\Sigma$ e consideraremos $\Sigma$ uma superfície de Riemann com estrutura complexa dada pela métrica induzida, e também $\partial_{z}=$ $\frac{1}{2}\left(\partial_{u}-i \partial_{v}\right)$ e $\partial_{\bar{z}}=\frac{1}{2}\left(\partial_{u}+i \partial_{v}\right)$. O conjunto $(\lambda, \nu, H, Q, t) \in \mathbb{R}^{+} \times[-1,1] \times \mathbb{R} \times \mathbb{C} \times \mathbb{C}$, será chamado de dados fundamentais de $\Sigma$, onde $\lambda$ é o fator de conformidade da métrica induzida, ou seja, $\lambda=2\left\langle\partial_{z}, \partial_{\bar{z}}\right\rangle, \nu=\langle N, \xi\rangle$ é a componente normal do campo vertical $\xi$ e $H$ é a curvatura média de $\Sigma$. Além disso, $Q$ é a diferencial de Hopf de $\Sigma$, isto é,

$$
Q d z^{2}=\left\langle-\bar{\nabla}_{\partial_{z}} N, \partial_{z}\right\rangle d z^{2}
$$

e $t=\left\langle\xi, \partial_{z}\right\rangle=\left\langle T, \partial_{z}\right\rangle$, com $T \in \mathfrak{X}(\Sigma)$ sendo a componente tangente do campo vertical $\xi$, ou seja, $T=\xi-\nu N$. A primeira e a segunda formas fundamentais são escritas em termos do parâmetro complexo, respectivamente, como 


$$
\begin{aligned}
I & =2 \lambda|d z|^{2} \\
I I & =Q d z^{2}+2 \lambda H|d z|^{2}+\bar{Q} d \bar{z}^{2}
\end{aligned}
$$

No próximo lema, serão apresentadas as equações fundamentais para a imersão e as condições de compatibilidade reescritas para o caso em que temos um parâmetro conforme para a primeira forma fundamental. Estas equações, que são equivalentes às de compatibilidade, são chamadas de condições de Integrabilidade.

Lema 4.2.2 (Ver [16]). Dada uma imersão $H$-superfície $\Sigma \subset \mathbb{E}(\kappa, \tau)$, as seguintes equações são satisfeitas

$$
\begin{aligned}
K & =K_{e}+\tau^{2}+\left(\kappa-4 \tau^{2}\right) \nu^{2} \\
Q_{\bar{z}} & =\lambda\left(\kappa-4 \tau^{2}\right) \nu t \\
t_{z} & =\frac{\lambda z}{\lambda} t+Q \nu \\
t_{\bar{z}} & =\lambda(H+i \tau) \nu \\
\nu_{z} & =-(H-i \tau) t-\frac{Q_{\bar{t}}}{\lambda} \\
|t|^{2} & =\frac{1}{2} \lambda\left(1-\nu^{2}\right)
\end{aligned}
$$

onde $t=\left\langle T, \partial_{z}\right\rangle, \bar{t}=\left\langle T, \partial_{\bar{z}}\right\rangle, K_{e}$ é a curvatura extrínseca e $K$ é a curvatura Gaussiana.

Em 2004 e 2005, Uwe Abresch e Harold Rosenberg ([1], [2]) generalizaram a diferencial de Hopf para superfícies imersas em $\mathbb{S}^{2} \times \mathbb{R}$ e $\mathbb{S}^{2} \times \mathbb{R}$. Mais geralmente, o fizeram para espaços homogêneos tridimensionais com grupo de isometria de dimensão quatro, mantendo a propriedade de ser holomorfa quando a curvatura média é constante, classificaram as imersões com essa diferencial nula e obtiveram um resultado correspondente ao de Hopf para superfícies imersas em $\mathbb{E}(\kappa, \tau)$.

Agora, definiremos a diferencial quadrática de Abresch-Rosenberg. Note que diferentemente do que acontece para imersões em $\mathbb{R}^{3}$, onde a diferencial de Hopf ser holomorfa implica que a superfície tem curvatura média constante, a diferencial de Abresch-Rosenberg pode ser holomorfa em alguma superfície cuja curvatura média não é constante. Todavia, a recíproca desta afirmação é valida em $\mathbb{E}(\kappa, \tau)$.

Considere uma $H$-superfície imersa $\Sigma \subset \mathbb{E}(\kappa, \tau)$. Então existe uma diferencial quadrática definida globalmente, chamada a diferencia de Abresch-Rosenberg.

Definição 4.2.1. Dado um parâmetro local conforme z, a diferencial de Abresch-Rosenberg é definida por

$$
\mathcal{Q}^{A R}=Q^{A R} d z^{2}=\left(2(H+i \tau) Q-\left(\kappa-4 \tau^{2}\right) t^{2}\right) d z^{2} .
$$


Além disso, associada a diferencial de Abresch-Rosenberg definimos a função AbreschRosenberg $q^{A R}: \Sigma \longrightarrow[0,+\infty]$ por

$$
q^{A R}=\frac{\left|Q^{A R}\right|}{\lambda^{2}}
$$

Note que $\mathcal{Q}^{A R}$ e $q^{A R}$ são globalmente definidas em $\Sigma$. Observe também que a diferencial (4.16) coincide com a diferencial usual de Hopf em $\mathbb{R}^{3}$ (isso ocorre quando $\kappa=0=\tau$ ) a menos de um fator constante. Agora veremos a prova que a diferencial da Definição 4.2.1 é holomorfa em qualquer $H$-superficies em $\mathbb{E}(\kappa, \tau)$

Teorema 4.2.3. Se $\Sigma$ é uma $H$-superfície em $\mathbb{E}(\kappa, \tau)$, então a diferencial Abresch-Rosenberg $\mathcal{Q}^{A R}$ é holomorfa para uma estrutura induzida pela primeira forma fundamental I.

Demonstração. A prova segue de (4.11) e (4.13) e o fato de $\Sigma$ ter curvatura média constante. Derivando (4.16) em relação a $\bar{z}$ temos:

$$
\begin{aligned}
Q_{\bar{z}}^{A R} & =2(H+i \tau) Q_{\bar{z}}-2\left(\kappa-4 \tau^{2}\right) t t_{\bar{z}} \\
& =2(H+i \tau) \lambda\left(\kappa-4 \tau^{2}\right) \nu t-2\left(\kappa-4 \tau^{2}\right) t \lambda(H+i \tau) \nu t \\
& =0
\end{aligned}
$$

\subsection{Diferencial Abresch-Rosenberg e Pares de Codazzi}

Definição 4.3.1. Sejam z um parâmetro conforme local e $(I, I I)$ um par fundamental em $\Sigma$ dados por

$$
\begin{aligned}
I & =2 \lambda|d z|^{2} \\
I I & =Q d z^{2}+2 \rho|d z|^{2}+\bar{Q} d \bar{z}^{2} .
\end{aligned}
$$

Dizemos que a forma quadrática $Q d z^{2}$ de II é a diferencial de Hopf do par (I, II). Em outras palavras, a (2,0)-parte de II é a diferencial de Hopf de $(I, I I)$.

Notemos que $(I, I I)$ é umbílico em $p \in \Sigma$ se, e somente se, $Q(p)=0$, isto é, a diferencial de Hopf determina a umbilicidade do par fundamental $(I, I I)$ nos pontos da superfície $\Sigma$.

Observação 4.3.1. Se considerarmos $\Sigma$ como uma superfície de Riemann com respeito à métrica I e z um parâmetro conforme, então podemos escrever

$$
\begin{aligned}
I & =2 \lambda|d z|^{2} \\
I I & =Q d z^{2}+2 H \lambda\left|d z^{2}\right|+\bar{Q} d \bar{z}^{2},
\end{aligned}
$$


na qual se verificam as seguintes equações:

$$
\begin{aligned}
K_{e} & =H^{2}-\frac{|Q|^{2}}{\lambda^{2}} \\
S \partial_{z} & =H \partial_{z}+\frac{Q \partial_{\bar{z}}}{\lambda} \\
\Gamma_{11}^{1} & =\frac{\lambda_{z}}{\lambda} \\
\Gamma_{11}^{2} & =\Gamma_{12}^{1}=0 \\
T_{S}\left(\partial_{z}, \partial_{\bar{z}}\right) & =T_{H}\left(\partial_{z}, \partial_{\bar{z}}\right)+\frac{1}{\lambda}\left(\bar{Q}_{\bar{z}} \partial_{z}+Q_{\bar{z}} \partial_{\bar{z}}\right) \\
\left\langle T_{S}\left(\partial_{z}, \partial_{\bar{z}}\right), \partial_{z}\right\rangle & =\lambda H_{z}-Q_{\bar{z}} \\
K(I) & =-\frac{1}{\lambda}(\ln \lambda)_{z \bar{z}}
\end{aligned}
$$

Um caso especialmente interessante ocorre quando o par fundamental satisfaz à equação de Codazzi, ou seja:

Lembremos que um par fundamental $(I, I I)$ em uma superfície $\Sigma$, com operador forma associado $S$, é um par de Codazzi se

$$
\nabla_{X} S Y-\nabla_{Y} S X-S[X, Y]=0 \quad X, Y \in \mathfrak{X}(\Sigma),
$$

onde $\nabla$ é a conexão de Levi Civita associado a métrica Riemanniana $I$.

Observemos também que o par fundamental $(I, I I)$ é um par de Codazzi se, e somente se, $Q_{\bar{z}}=\lambda H_{z}$. Assim, temos:

Lema 4.3.1 (Versão Abstrata de Hopf). Seja (I,II) par fundamental. Então quaisquer duas das seguintes condições implicam na terceira:

(i) $(I, I I)$ é um par de Codazzi;

(ii) $H$ é constante;

(iii) A diferencial de Hopf é holomorfa.

Em particular, se verificam duas das condiçôes anteriores e $\Sigma$ é uma esfera topológica, o par tem que ser totalmente umbílico.

Demonstração. Seja z um parâmetro conforme local para $I$, o Tensor de Codazzi associado a $(I, I I)$, satisfaz

$$
T_{S}\left(\partial_{z}, \partial_{\bar{z}}\right)=T_{H}\left(\partial_{z}, \partial_{\bar{z}}\right)+\frac{1}{\lambda}\left(\bar{Q}_{z} \partial_{z}+Q_{\bar{z}} \partial_{\bar{z}}\right) .
$$

Agora, o resultado é consequência direta das seguintes equivalências:

- $(I, I I)$ é Codazzi se, e somente se, $Q_{\bar{z}}=\lambda H_{z}$;

- $H$ é constante se, e somente se, $T_{H}=0$ 
- $Q$ é holomorfa se, e somente se, $Q_{\bar{z}}=0$.

Até agora vimos que a diferencial quadrática $\mathcal{Q}^{A R}$ dada na Definição 4.2.1 é holomorfa em qualquer $H$-superfície $\Sigma$ imersa em $\mathbb{E}(\kappa, \tau)$. Consequentemente, é fácil ver que tal diferencial quadrática deve ser nula em uma esfera topológica pelo Lema 4.3.1. Em seguida Abresch e Rosenberg mostraram que se a diferencial Abresch Rosenberg se anula em uma H-superfície então essa deve ser invariante sob um subgrupo a um parâmetro de isometria da variedade ambiente $\mathbb{E}(\kappa, \tau)$. Em particular, quando a superfície é uma esfera topológica, essa deve ser rotacionalmente simétrica.

Especificamente eles provaram:

Teorema 4.3.1. Qualquer esfera topológica em $\mathbb{E}(\kappa, \tau)$ com curvatura média constante $H$ é uma esfera rotacionalmente simétrica.

O Lema 4.3.1 nos diz que a existência de uma diferencial quadrática implica na existência de um par Codazzi sobre qualquer $H$-superfície em $\mathbb{E}(\kappa, \tau)$. Quando $\mathbb{E}(\kappa, \tau)$ é uma variedade produto, isto é, $\tau=0$, tal par Codazzi foi encontrado um longo tempo depois (Ver [4]).

Agora estudaremos um par de Codazzi em qualquer $H$-superfície tal que a diferencial de Abresch-Rosenberg aparece como sua diferencial de Hopf.

Primeiramente, recorremos ao caso $\tau=0$, uma vez que auxiliará o caso $\tau \neq 0$.

\subsubsection{H-Superfícies Imersas em $\mathbb{E}(\kappa, \tau) \operatorname{com} \tau=0$}

Nesta seção, vamos estudar brevemente como alguns pares Codazzi aparecem no contexto de $H$-superfícies. Considere uma superfície $\Sigma$ e uma imersão $\psi: \Sigma \longrightarrow \mathbb{M}^{2} \times \mathbb{R}$, com vetor normal e considere a função altura $h:=\pi \circ \psi$ e a função ângulo $\nu:=d \pi(N)$ na superfície $\Sigma$.

Se $\psi$ é uma imersão com curvatura média constante $H$, então foi provado por Abresch e Rosenberg [1], que a diferencial quadrática dada na Definição 4.2.1 quando $\tau=0$ é holomorfa, ou seja, $\left(2 H Q-\varepsilon h_{z}^{2}\right) d z^{2}$ é holomorfa. Aqui $z$ é um parâmetro conforme para a métrica induzida $I$ e $Q d z^{2}$ é a diferencial de Hopf para o par $(I, I I)$.

Notemos que $\mathcal{Q} d z^{2}=\left(2 H Q-\varepsilon h_{z}^{2}\right) d z^{2}$ é a $(2,0)$ - parte da forma quadrática $B=2 H I-\varepsilon d h^{2}+$ $\frac{\varepsilon}{2}\|\nabla h\|^{2} I$ para a estrutura conforme dada por $I$ (ver [4]).

Teorema 4.3.2. Seja $\Sigma \subset \mathbb{M}^{2}(\varepsilon) \times \mathbb{R}$ uma superfície imersa com curvatura constante $H$. Então $(I, B)$ é um par de Codazzi com curvatura média constante $2 H^{2}$.

Demonstração. Mostraremos que $H(I, B)=2 H^{2}$. 
Sabemos que

$$
H(I, I I)=\frac{e G-2 f F+E g}{2\left(E G-F^{2}\right)} .
$$

Denotemos

$$
H(I, B)=\frac{e_{B} G-2 f_{B} F+E g_{B}}{2\left(E G-F^{2}\right)} .
$$

Como $B=2 H I I+\varepsilon d h^{2}+\frac{\varepsilon}{2}\|\nabla h\|^{2} I$, então

$$
\begin{aligned}
H(I, B) & =\frac{\left(2 H e+\varepsilon h_{u}^{2}+\frac{\varepsilon}{2}\|\nabla h\|^{2} E\right) G}{2\left(E G-F^{2}\right)} \\
& -\frac{2 F\left(2 H f+\varepsilon h_{u} h_{v}+\frac{\varepsilon}{2}\|\nabla h\|^{2} E\right)}{2\left(E G-F^{2}\right)} \\
& +\frac{E\left(2 H g+\varepsilon h_{v}^{2}+\frac{\varepsilon}{2}\|\nabla\| \|^{2} G\right)}{2\left(E G-F^{2}\right)}
\end{aligned}
$$

Reorganizando

$$
\begin{aligned}
H(I, B) & =\frac{2 H(e G-2 F f+E g)+\frac{\varepsilon}{2}\|\nabla h\|^{2}\left(E G-2 F^{2}+E G\right)}{2\left(E G-F^{2}\right)} \\
& -\frac{\varepsilon\left(G h_{u}^{2}-2 F h_{u} h_{v}+E h_{v}^{2}\right)}{2\left(E G-F^{2}\right)} .
\end{aligned}
$$

Logo

$$
H(I, B)=\frac{4 H^{2}\left(E G-F^{2}\right)+\varepsilon\|\nabla h\|^{2}\left(E G-F^{2}\right)-\varepsilon\left(G h_{u}^{2}-2 h_{u} h_{v} F+E h_{v}^{2}\right)}{2\left(E G-F^{2}\right)} .
$$

Então, segue que

$$
H(I, B)=2 H^{2}+\frac{\varepsilon}{2}\left[\|\nabla h\|^{2}-\left(\frac{G h_{u}^{2}+2 E h_{u} h_{v} F+E h_{v}^{2}}{E G-F^{2}}\right)\right] .
$$

Iremos considerar a partir de agora parâmetros ortogonais, isto é, $F=f=0$ para facilitar nossos cálculos. Assim, temos

$$
H(I, B)=2 H^{2}+\frac{\varepsilon}{2}\left[\|\nabla h\|^{2}-\left(\frac{G h_{u}^{2}+E h_{v}^{2}}{E G}\right)\right] .
$$

Sabemos

$$
\nabla f=\frac{\left(G f_{u}-F f_{v}\right) \partial_{u}+\left(E f_{v}-F f_{u}\right) \partial_{v}}{E G-F^{2}} .
$$

Então, segue daí e da parametrização ortogonal, que

$$
\|\nabla h\|^{2}=\frac{G h_{u}^{2}+E h_{v}^{2}}{E G} .
$$


Logo,

$$
H(I, B)=2 H^{2}
$$

Portanto, se a curvatura média do par $(I, B)$ é dada por $H(I, B)=2 H^{2}$, então $(I, B)$ é um par de Codazzi pelo Lema 4.3.1, uma vez que $H(I, B)$ é constante e a diferencial de Hopf é holomorfa.

\subsubsection{H-Superfícies Imersas em $\mathbb{E}(\kappa, \tau)$ com $\tau \neq 0$}

A diferencial de Abresch-Rosenberg tem outra interpretação em termos de pares de Codazzi definida em qualquer $H$-superfície em $\mathbb{E}(\kappa, \tau)$ quando $\tau \neq 0$. Assuma que $H^{2}+\tau^{2}>0$. Defina $\theta \in[0,2 \pi]$ por

$$
e^{2 \theta i}=\frac{H-i \tau}{\sqrt{h^{2}+\tau^{2}}}
$$

Seja $\Sigma \subset \mathbb{E}(\kappa, \tau)$ uma $H$-superfície e considere $z$ um parâmetro conforme local. Então, a menos de uma constante complexa $H+i \tau$, podemos redefinir a diferencial de Abresch-Rosenberg como

$$
Q^{A R} d z^{2}=\left(Q-\frac{\kappa-4 \tau^{2}}{2(H+i \tau)} t^{2}\right) d z^{2}
$$

Reescrevendo a diferencial acima como:

$$
Q^{A R} d z^{2}=\left(Q-\frac{\kappa-4 \tau^{2}}{2(H+i \tau)}\left(e^{\theta i} t\right)^{2}\right) d z^{2}
$$

Dado um campo de vetores tangentes $T$, definimos

$$
T_{\theta}=\cos \theta T+\sin \theta J T
$$

onde, recordando, $J X=N \wedge X, \forall X \in \mathfrak{X}(\Sigma)$, em que $N$ denota o vetor normal unitário ao longo de $\Sigma$.

Então, podemos facilmente checar que $\left\langle T_{\theta}, \partial_{z}\right\rangle=e^{i \theta} t$. Logo,

$$
Q^{A R} d z^{2}=\left(\left\langle A \partial_{z}, \partial_{z}\right\rangle-\alpha\left\langle T_{\theta}, \partial_{z}\right\rangle^{2}\right) d z^{2}
$$

onde $\alpha=\frac{\kappa-4 \tau^{2}}{2 \sqrt{H^{2}+\tau^{2}}} \in \mathbb{R}$ e $A$ é o operador forma associado a $N$, tal que, a segunda forma fundamental é dada por $I I(X, Y)=\langle A X, Y\rangle, X, Y \in \mathfrak{X}(\Sigma)$. Isto nos leva a seguinte definição: 
Definição 4.3.2. Dada uma $H$-superfície $\Sigma \subset \mathbb{E}(\kappa, \tau)$ a segunda forma fundamental Abresch-Rosenberg é definida por

$$
I I_{A R}(X, Y)=I I(X, Y)-\alpha\left\langle T_{\theta}, X\right\rangle\left\langle T_{\theta}, Y\right\rangle+\frac{\alpha|T|^{2}}{2}\langle X, Y\rangle,
$$

ou equivalentemente, o operador forma Abresch-Rosenberg $S_{A R}$ é definido por

$$
S_{A R} X=A(X)-\alpha\left\langle T_{\theta}, X\right\rangle T_{\theta}+\frac{\alpha|T|^{2}}{2} X
$$

ou, o traço do operador forma Abresch-Rosenberg $S$ é definido por

$$
S X=S_{A R} X-H X=A(X)-\alpha\left\langle T_{\theta}, X\right\rangle T_{\theta}+\frac{\alpha|T|^{2}}{2} X-H X
$$

onde $X, Y \in \mathfrak{X}(\Sigma)$.

Primeiro, examinaremos as propriedades geométricas da forma quadrática acima e sua relação com a diferencial Abresch-Rosenberg.

Antes de provamos nosso próximo resultado faremos uma breve observação na qual mostraremos que $q^{A R}$ é a curvatura assimétrica do par $\left(I, I I_{A R}\right)$.

Observação 4.3.2. Por definição, sabemos que

$$
q^{A R}=\frac{\left|Q^{A R}\right|}{\lambda^{2}}
$$

Mas note que da equação (4.17) da Observação 4.3.1 temos que

$$
\frac{\left|Q^{A R}\right|^{2}}{\lambda^{2}}=H_{A R}^{2}-K_{e}\left(I, I I_{A R}\right)
$$

Logo

$$
q^{A R}=H_{A R}^{2}-K_{e}\left(I, I I_{A R}\right) .
$$

Portanto, $q^{A R}$ é a curvatura assimétrica do par $\left(I, I I_{A R}\right)$.

Proposição 4.3.1. Valem as seguintes equações para o par fundamental $\left(I, I I_{A R}\right)$ :

1) $I I_{A R}\left(\partial_{z}, \partial_{z}\right) d z^{2}=Q^{A R} d z^{2}$, onde z é um parâmetro conforme local para $I$.

2) $H\left(I, I I_{A R}\right)=H(I, I I)$;

3) $K_{e}\left(I, I I_{A R}\right)=K_{e}(I, I I)+\alpha\left\langle S T_{\theta}, T_{\theta}\right\rangle+\frac{\alpha^{2}|T|^{4}}{4}$.

Além disso, a norma quadrada do operador forma $|A|^{2}$ e a norma quadrada do traço do operador forma Abresch-Rosenberg $|S|^{2}$ satisfazem

$$
|A|^{2}=|S|^{2}+2 \alpha\left\langle S T_{\theta}, T_{\theta}\right\rangle+\frac{\alpha^{2}}{2}|T|^{4}+2 H^{2}
$$


mais ainda,

$$
\frac{|T|^{4}}{2}-\frac{\left\langle S T_{\theta}, T_{\theta}\right\rangle}{|S|^{2}}=\frac{\left\langle S T_{\theta}, J T_{\theta}\right\rangle}{|S|^{2}}
$$

Demonstração. Primeiramente notemos

$$
\left|T_{\theta}\right|=\left|J T_{\theta}\right|=|T|
$$

De fato,

$$
\left|J T_{\theta}\right|^{2}=\left\langle N \wedge T_{\theta}, N \wedge T_{\theta}\right\rangle=\left\langle T_{\theta},\left(N \wedge T_{\theta}\right) \wedge N\right\rangle=\left\langle T_{\theta}, T_{\theta}\right\rangle=\left|T_{\theta}\right|^{2}
$$

$\mathrm{e}$

$$
\left|T_{\theta}\right|^{2}=\cos ^{2} \theta|T|^{2}+\sin ^{2} \theta|J T|^{2}+2 \sin \theta \cos \theta\langle T, J T\rangle=|T|^{2} .
$$

Agora considere um parâmetro conforme local $z$ para $I$. Então temos que $I\left(\partial_{z}, \partial_{z}\right)=p=0$. Fazendo $X=Y=\partial_{z}$ em (4.24) segue que

$$
I I_{A R}\left(\partial_{z}, \partial_{z}\right)=I I\left(\partial_{z}, \partial_{z}\right)-\alpha\left\langle T_{\theta}, \partial_{z}\right\rangle\left\langle T_{\theta}, \partial_{z}\right\rangle+\frac{\alpha|T|^{2}}{2}\left\langle\partial_{z}, \partial_{z}\right\rangle,
$$

ou seja,

$$
I I_{A R}\left(\partial_{z}, \partial_{z}\right) d z=\left(I I\left(\partial_{z}, \partial_{z}\right)-\alpha\left\langle T_{\theta}, \partial_{z}\right\rangle^{2}\right) d z
$$

Para mostrar que $H(I, I I)=H\left(I, I I_{A R}\right)$ basta provar que $\operatorname{tr} A=\operatorname{tr} S_{A R}$.

Sendo $\left\{e_{1}, e_{2}\right\}$ base ortonormal, sabemos que

$$
\operatorname{tr} S_{A R}=\left\langle S_{A R} e_{1}, e_{1}\right\rangle+\left\langle S_{A R} e_{2}, e_{2}\right\rangle .
$$

Tomando $\left\{e_{1}, e_{2}\right\}$ vetores principais de $A$, segue de (4.25) que

$$
S_{A R} e_{1}=A\left(e_{1}\right)-\alpha\left\langle T_{\theta}, e_{1}\right\rangle T_{\theta}+\frac{\alpha|T|^{2}}{2} e_{1}
$$

Logo

$$
\left\langle S_{A R} e_{1}, e_{1}\right\rangle=\left\langle A e_{1}, e_{1}\right\rangle-\alpha\left\langle T_{\theta}, e_{1}\right\rangle^{2}+\frac{\alpha|T|^{2}}{2} .
$$


De modo análogo, obtemos

$$
\left\langle S_{A R} e_{2}, e_{2}\right\rangle=\left\langle A e_{2}, e_{2}\right\rangle-\alpha\left\langle T_{\theta}, e_{2}\right\rangle^{2}+\frac{\alpha|T|^{2}}{2} .
$$

Consequentemente

$$
\operatorname{Tr} S_{A R}=\left\langle A e_{1}, e_{1}\right\rangle+\left\langle A e_{2}, e_{2}\right\rangle-\alpha\left[\left\langle T_{\theta}, e_{1}\right\rangle^{2}+\left\langle T_{\theta}, e_{2}\right\rangle^{2}\right]+\alpha|T|^{2},
$$

isto é,

$$
\operatorname{Tr} S_{A R}=\operatorname{Tr} A-\alpha\left[\left\langle T_{\theta}, e_{1}\right\rangle^{2}+\left\langle T_{\theta}, e_{2}\right\rangle^{2}\right]+\alpha|T|^{2} .
$$

Escrevendo $T_{\theta}=\theta_{1} e_{1}+\theta_{2} e_{2}$, obtemos

$$
\theta_{1}=\left\langle T_{\theta}, e_{1}\right\rangle \quad \text { e } \quad \theta_{2}=\left\langle T_{\theta}, e_{2}\right\rangle .
$$

Note que $\left|T_{\theta}\right|^{2}=\theta_{1}^{2}+\theta_{2}^{2}$, logo

$$
\left|T_{\theta}\right|^{2}=\left\langle T_{\theta}, e_{1}\right\rangle^{2}+\left\langle T_{\theta}, e_{2}\right\rangle^{2}
$$

Como $|T|=\left|T_{\theta}\right|$ temos

$$
|T|^{2}=\left\langle T_{\theta}, e_{1}\right\rangle^{2}+\left\langle T_{\theta}, e_{2}\right\rangle^{2}
$$

Deste modo, $\operatorname{Tr} S_{A R}=\operatorname{Tr} A$. Portanto,

$$
H\left(I, I I_{A R}\right)=H(I, I I)
$$

Observe que chegaríamos neste mesmo resultado usando o fato de que $S$ é o operador sem traço de Abresch-Rosenberg.

Agora calculemos $K_{e}\left(I, I I_{A R}\right)$. Segue de (4.29) que

$$
\begin{aligned}
I I_{A R}\left(T_{\theta}, T_{\theta}\right) & =I I\left(T_{\theta}, T_{\theta}\right)-\alpha\left|T_{\theta}\right|^{4}+\frac{\alpha\left|T_{\theta}\right|^{4}}{2} \\
I I_{A R}\left(T_{\theta}, J T_{\theta}\right) & =I I\left(T_{\theta}, J T_{\theta}\right) \\
I I_{A R}\left(J T_{\theta}, J T_{\theta}\right) & =I I\left(J T_{\theta}, J T_{\theta}\right)+\frac{\alpha|T|}{2} .
\end{aligned}
$$

Pela definição da forma quadrática Abresch-Rosenberg, temos que $K_{e}\left(I, I I_{A R}\right)=K_{e}(I, I I)$ em um conjunto $U=\left\{p \in \Sigma ;|T|^{2}(p)=0\right\}$. Então, considerando $p \in \Sigma \backslash U$ e escolhendo uma base em $T_{p} \Sigma$ definida por

$$
e_{1}=\frac{T_{\theta}}{|T|} \quad \text { e } \quad e_{2}=\frac{J T_{\theta}}{|T|}
$$


de (4.24), segue que

$$
\begin{aligned}
& I I_{A R}\left(e_{1}, e_{1}\right)-I I_{A R}\left(e_{2}, e_{2}\right)=I I\left(e_{1}, e_{1}\right)-\alpha\left\langle T_{\theta}, \frac{T_{\theta}}{|T|}\right\rangle^{2}+\frac{\alpha|T|^{2}}{2}\left\langle\frac{T_{\theta}}{|T|}, \frac{T_{\theta}}{|T|}\right\rangle \\
& -I I\left(e_{2}, e_{2}\right)+\alpha\left\langle T_{\theta}, \frac{J T_{\theta}}{|T|^{2}}\right\rangle^{2}-\frac{\alpha|T|^{2}}{2}\left\langle\frac{J T_{\theta}}{|T|}, \frac{J T_{\theta}}{|T|}\right\rangle \\
& =I I\left(e_{1}, e_{1}\right)-\frac{\alpha}{|T|^{2}}\left\langle T_{\theta}, T_{\theta}\right\rangle^{2}+\frac{\alpha}{2}\left\langle T_{\theta}, T_{\theta}\right\rangle-I I\left(e_{2}, e_{2}\right)-\frac{\alpha|T|^{2}}{2|T|^{2}}\left\langle J T_{\theta}, J T_{\theta}\right\rangle \\
& =I I\left(e_{1}, e_{1}\right)-\alpha|T|^{2}+\frac{\alpha|T|^{2}}{2}-I I\left(e_{2}, e_{2}\right)-\frac{\alpha}{2}\left\langle J T_{\theta}, J T_{\theta}\right\rangle \\
& =I I\left(e_{1}, e_{1}\right)-\frac{\alpha}{2}|T|^{2}-I I\left(e_{2}, e_{2}\right)-\frac{\alpha}{2}\left\langle J T_{\theta}, J T_{\theta}\right\rangle \\
& =I I\left(e_{1}, e_{1}\right)-I I\left(e_{2}, e_{2}\right)-\alpha|T|^{2}
\end{aligned}
$$

e seja $\left\{e_{1}, e_{2}\right\}$ ortogonal em $p$, então

$$
\begin{aligned}
K_{e}\left(I, I I_{A R}\right)= & I I_{A R}\left(e_{1}, e_{1}\right) I I_{A R}\left(e_{2}, e_{2}\right)-I I_{A R}^{2}\left(e_{1}, e_{2}\right) \\
= & \left(I I\left(e_{1}, e_{1}\right)-\frac{\alpha}{2}|T|^{2}\right)\left(I I\left(e_{2}, e_{2}\right)+\frac{\alpha}{2}|T|^{2}\right)-I I^{2}\left(e_{1}, e_{2}\right) \\
= & I I\left(e_{1}, e_{1}\right) I I\left(e_{2}, e_{2}\right)+I I\left(e_{1}, e_{1}\right) \frac{\alpha|T|^{2}}{2}-I I\left(e_{2}, e_{2}\right) \frac{\alpha|T|^{2}}{2} \\
& -\frac{\alpha^{2}}{4}|T|^{4}-I I\left(e_{1}, e_{2}\right) \\
= & I I\left(e_{1}, e_{1}\right) I I\left(e_{2}, e_{2}\right)-I I\left(e_{1}, e_{1}\right)^{2} \\
& \left.+\frac{\alpha}{2}\left(I I\left(e_{1}, e_{2}\right)\right)-I I\left(e_{2}, e_{2}\right)|T|^{2}\right)-\frac{\alpha^{2}}{4}|T|^{4} .
\end{aligned}
$$

Por outro lado, substituindo (4.31) na fórmula acima e simplificando termos, conseguimos em $p$

$$
\begin{aligned}
& K_{e}\left(I, I I_{A R}\right)=K_{e}(I, I I)+\frac{\alpha}{2}\left(I I_{A R}\left(e_{1}, e_{1}\right)-I I_{A R}\left(e_{2}, e_{2}\right)+\alpha|T|^{2}\right)|T|^{2}-\frac{\alpha^{2}}{4}|T|^{4} \\
& \left.=K_{e}(I, I I)+\frac{\alpha}{2}\left(\frac{1}{|T|^{2}} I I_{A R}\left(T_{\theta}, T_{\theta}\right)-\frac{1}{|T|^{2}} I I_{A R}\left(J T_{\theta}, J T_{\theta}\right)+\alpha|T|^{2}\right)\right)|T|^{2}-\frac{\alpha^{2}}{4}|T|^{4} \\
& =K_{e}(I, I I)+\frac{\alpha}{2}\left(I I_{A R}\left(T_{\theta}, T_{\theta}\right)-I I_{A R}\left(J T_{\theta}, J T_{\theta}\right)\right)+\frac{\alpha^{2}}{2}|T|^{4}-\frac{\alpha^{2}}{4}|T|^{4} \\
& =K_{e}(I, I I)+\frac{\alpha}{2}\left(I I_{A R}\left(T_{\theta}, T_{\theta}\right)-I I_{A R}\left(J T_{\theta}, J T_{\theta}\right)\right)+\frac{\alpha^{2}}{4}|T|^{4} .
\end{aligned}
$$

Agora, recordando que $S$ possui traço nulo e assim, em um ponto $p \in \Sigma$, podemos considerar uma base ortonormal $\left\{e_{1}, e_{2}\right\}$, das direções principais de $S$, isto é,

$$
S e_{1}=k e_{1}, \quad S e_{2}=-k e_{2} \quad \text { e } \quad|S|^{2}=2 k^{2} .
$$


Então, existe $\beta \in[0,2 \pi)$ tal que

$$
T_{\theta}=|T|\left((\cos \beta) \frac{T}{|T|}+(\sin \beta) \frac{J T}{|T|}\right),
$$

ou seja, $T_{\theta}=|T|\left((\cos \beta) e_{1}+(\sin \beta) e_{2}\right)$.

Então $S T_{\theta}=|T| k\left((\cos \beta) e_{1}-(\sin \beta) e_{2}\right)$.

Agora notemos que

$$
T_{\theta}=(\cos \theta) T+(\sin \theta) J T .
$$

Daí $J T_{\theta}=(\cos \theta) J T-(\sin \theta) T$. Na nova base, temos

$$
\begin{array}{r}
J T_{\theta}=|T|\left[(\cos \beta) \frac{J T}{|T|}-(\sin \beta) \frac{T}{|T|}\right] \\
=|T|\left[(\cos \beta) e_{2}-(\sin \beta) e_{1}\right] .
\end{array}
$$

Assim $S J T_{\theta}=|T| k\left[(\cos \beta) e_{2}+(\sin \beta) e_{1}\right]$. Segue que

$$
\left\langle S T_{\theta}, T_{\theta}\right\rangle=|T|^{2} k\left[\cos ^{2} \beta-\sin ^{2} \beta\right] \quad \text { e } \quad\left\langle S J T_{\theta}, J T_{\theta}\right\rangle=-|T|^{2} k\left[\cos ^{2} \beta-\sin ^{2} \beta\right] .
$$

Isto é,

$$
\left\langle S T_{\theta}, T_{\theta}\right\rangle=-\left\langle S J T_{\theta}, J T_{\theta}\right\rangle
$$

Então, de (4.26) e (4.33) temos

$$
I I_{A R}\left(T_{\theta}, T_{\theta}\right)-I I_{A R}\left(J T_{\theta}, J T_{\theta}\right)=\left\langle S T_{\theta}, T_{\theta}\right\rangle-\left\langle S J T_{\theta}, T_{\theta}\right\rangle=2\left\langle S T_{\theta}, T_{\theta}\right\rangle .
$$

Assim, substituindo a igualdade acima na equação (4.32) temos a expressão de $K_{e}\left(I, I I_{A R}\right)$ que queríamos.

Além disso, por meio de um calculo simples, usando (4.33) temos que

$$
\left\langle S T_{\theta}, T_{\theta}\right\rangle^{2}+\left\langle S T_{\theta}, J T_{\theta}\right\rangle^{2}=|T|^{4} k^{2}=\frac{|S|^{2}}{2}|T|^{4} .
$$

Logo,

$$
\frac{|T|^{4}}{2}-\frac{\left\langle S T_{\theta}, T_{\theta}\right\rangle^{2}}{|S|^{2}}=\frac{\left\langle S T_{\theta}, J T_{\theta}\right\rangle^{2}}{|S|}
$$

o que mostra (4.28).

Afirmação 4.3.1. $|A|^{2}=4 H^{2}-2 K_{e} \quad e \quad|S|^{2}=2 q^{A R}=2\left(H^{2}-K_{e}\left(I, I I_{A R}\right)\right)$ 
Com efeito, a primeira igualdade segue do fato de que

$$
|A|^{2}=\left|A e_{1}\right|^{2}+\left|A e_{2}\right|^{2}=k_{1}+k_{2}=4 H^{2}-2 K_{e}
$$

A segunda segue de $S X=S_{A R} X-H X$. Consideremos $v_{i}$ autovetor de $S_{A R}$ e $H=\frac{k_{1}+k_{2}}{2}=$ $H_{A R}$. Notemos que

$$
\begin{aligned}
S v_{1} & =S_{A R} v_{1}-H v_{1} \\
& =k_{1} v_{1}-\left(\frac{k_{1}+k_{2}}{2}\right) v_{1} \\
& =\left(\frac{k_{1}-k_{2}}{2}\right) v_{1} .
\end{aligned}
$$

Analogamente,

$$
S v_{1}=\left(\frac{k_{2}-k_{1}}{2}\right) v_{2}
$$

Ou seja, se $v_{i}$ autovetor de $S_{A R}$, então $v_{i}=e_{i}$ e assim concluímos

$$
k=\frac{k_{1}-k_{2}}{2} \quad e \quad-k=\frac{k_{2}-k_{1}}{2} .
$$

Temos $|S|^{2}=2 k^{2}$, então

$$
|S|^{2}=2\left(\frac{k_{1}-k_{2}}{2}\right)^{2}=2 \frac{\left(k_{1}-k_{2}\right)^{2}}{4} .
$$

Como $q^{A R}$ é a curvatura assimétrica, então

$$
|S|^{2}=2 q^{A R}=2\left(H^{2}-K_{e}\left(I, I I_{A R}\right)\right)
$$

concluindo assim a prova da afirmação. Na última igualdade usamos a Observação 4.3 .1 e o fato de $H(I, I I)=H_{A R}$

Para mostrar (4.27), basta aplicar a afirmação em

$$
K_{e}\left(I, I I_{A R}\right)=K_{e}(I, I I)+\alpha\left\langle S T_{\theta}, T_{\theta}\right\rangle+\frac{\alpha^{2}|T|^{4}}{4} .
$$

Na prática, notemos que a igualdade acima pode ser reescrita como

$$
-K_{e}(I, I I)=-K_{e}\left(I, I I_{A R}\right)+\alpha\left\langle S T_{\theta}, T_{\theta}\right\rangle+\frac{\alpha^{2}|T|^{4}}{4} .
$$

Agora, multiplicando por 2 ambos os membros da igualdade, temos

$$
-2 K_{e}(I, I I)=-2 K_{e}\left(I, I I_{A R}\right)+2 \alpha\left\langle S T_{\theta}, T_{\theta}\right\rangle+\frac{\alpha^{2}|T|^{4}}{2} .
$$


Somando $4 H^{2}$, segue que

$$
4 H^{2}-2 K_{e}(I, I I)=4 H^{2}-2 K_{e}\left(I, I I_{A R}\right)+2 \alpha\left\langle S T_{\theta}, T_{\theta}\right\rangle+\frac{\alpha^{2}|T|^{4}}{2},
$$

$\log 0$

$$
4 H^{2}-2 K_{e}(I, I I)=2 H^{2}-2 K_{e}\left(I, I I_{A R}\right)+2 \alpha\left\langle S T_{\theta}, T_{\theta}\right\rangle+\frac{\alpha^{2}|T|^{4}}{2}+2 H^{2} .
$$

Pela afirmação

$$
|A|^{2}=|S|^{2}+2 \alpha\left\langle S T_{\theta}, T_{\theta}\right\rangle+\frac{\alpha^{2}|T|^{4}}{2}+2 H^{2}
$$

Decorre da proposição anterior e do Lema 4.3 .1 o seguinte resultado:

Corolário 4.3.1. Dada qualquer $H$-superfície em $\mathbb{E}(\kappa, \tau), H^{2}+\tau^{2} \neq 0$ segue que $\mathcal{Q}^{A R}$ é holomorfa se, e somente se, $\left(I, I I_{A R}\right)$ é um par de Codazzi.

Assim, podemos resumir a discussão acima no teorema a seguir:

Teorema 4.3.3. Dada uma $H$-superfície $\Sigma \subset \mathbb{E}(\kappa, \tau), H^{2}+\tau^{2} \neq 0$, considere o $(2,0)$-tensor simétrico dado por

$$
I I_{A R}(X, Y)=I I(X, Y)-\alpha\left\langle T_{\theta}, X\right\rangle\left\langle T_{\theta}, Y\right\rangle+\frac{\alpha|T|^{2}}{2}\langle X, Y\rangle
$$

onde

$$
\begin{aligned}
\alpha & =\frac{\kappa-4 \tau^{2}}{2 \sqrt{H^{2}+\tau^{2}}} \in \mathbb{R} \\
e^{2 \theta i} & =\frac{H-i \tau}{\sqrt{h^{2}+\tau^{2}}} \\
T_{\theta} & =\cos \theta T+\sin \theta J T .
\end{aligned}
$$

Então, $\left(I, I I_{A R}\right)$ é um par de Codazzi com curvatura média constante H. Além disso, a $(2,0)$-parte de $I I_{A R}$ com respeito à estrutura conforme dada por I assemelha (a menos de uma constante) com a diferencial Abresch-Rosenberg.

\subsection{Classificação para H-Superfícies Imersas em $\mathbb{E}(\kappa, \tau)$}

Nosso propósito nesta seção é classificar $H$-superfície completa com função Abresch-Rosenberg constante não nula.

Lema 4.4.1 ([1]). Seja $\Sigma \subset \mathbb{E}(\kappa, \tau)$ uma $H$-superfície completa cuja diferencial AbreschRosenberg é nula. Então $\Sigma$ é um plano em $\mathbb{H}^{2} \times \mathbb{R}$ ou $\mathbb{S}^{2} \times \mathbb{R}$ se $H=0=\tau$, ou $\Sigma$ é invariante por um certo parâmetro subgrupo de isometria de $\mathbb{E}(\kappa, \tau)$. 
O lema acima motiva a seguinte definição:

Definição 4.4.1. Seja $\Sigma$ uma $H$-superfície completa em $\mathbb{E}(\kappa, \tau)$. Dizemos que $\Sigma$ é uma superfície Abresch-Rosenberg se sua diferencial Abresch-Rosenberg é identicamente nula.

Desde $\mathbb{E}(\kappa, \tau)$ é uma submersão Riemanniana $\pi: \mathbb{E}(\kappa, \tau) \longrightarrow \mathbb{M}^{2}(\kappa)$, dada $\gamma$ uma curva regular em $\mathbb{M}^{2}(\kappa), \Sigma_{\gamma}:=\pi^{-1}(\gamma)$ é uma superfície em $\mathbb{E}(\kappa, \tau)$ satisfazendo que $\xi$ é um campo vetorial tangente ao longo de $\Sigma_{\gamma}$, neste caso $\nu=0$. Então, $\xi$ é um campo vetorial paralelo ao longo de $\Sigma_{\gamma}$, e assim, $\Sigma_{\gamma}$ é flat e sua curvatura média é dada por $2 H=k_{g}$, onde $k_{g}$ é a curvatura geodésica de $\gamma$ em $\mathbb{M}^{2}(\kappa)(\operatorname{Ver}[18])$.

Definição 4.4.2. Dizemos que $\Sigma \subset \mathbb{E}(\kappa, \tau)$ é um cilindro de Hopf sobre $\gamma$, uma curva em $\mathbb{M}^{2}(\kappa)$ se $\Sigma_{\gamma}:=\pi^{-1}(\gamma)$. Isto é, se $\gamma$ é uma curva fechada, $\Sigma_{\gamma}$ é um cilindro Hopf flat $e$ adicionalmente, se $\pi$ é uma submersão de um círculo Riemanniano, $\Sigma_{\gamma}$ é um toro Hopf.

Agora, com a definição de cilindro Hopf, classificaremos $H$-superfícies em $\mathbb{E}(\kappa, \tau)$ com função Abresch-Rosenberg $q^{A R}$ constante não nula.

Antes de apresentar nosso último teorema, iremos enunciar definições e alguns resultados que nos serão úteis.

Lema 4.4.2. Seja $(\Sigma, I)$ uma superfície Riemanniana orientada e suponha que a diferencial quadrática $\mathcal{Q} \in \mathcal{Q}(\Sigma)$ é holomorfa. Seja z um parâmetro conforme local para $I, I=2 \lambda|d z|^{2}$, e seja $q^{A R}=\frac{|Q|^{2}}{\lambda^{2}}$, onde $\mathcal{Q}=Q d z^{2}$. Então

$$
\Delta \ln q^{A R}=4 K .
$$

Demonstração. Seja $z$ um parâmetro local conforme para $I$, então temos $I=2 \lambda\left|d z^{2}\right|$. Escrevendo $\mathcal{Q}=Q d z^{2}$ e seja $p \in \Sigma$ tal que $\mathcal{Q}(p) \neq 0$, então

$$
\Delta \ln q^{A R}=\Delta \ln q^{A R}=\frac{|Q|^{2}}{\lambda^{2}}=\Delta \ln |Q|^{2}-2 \Delta \ln \lambda .
$$

Sabemos que, em parâmetro conforme segue que

$$
\Delta f=\frac{2}{\lambda} f_{z \bar{z}}
$$

Notemos que do fato de $Q$ ser holomorfa temos

$$
\Delta \ln |Q|^{2}=\frac{2}{\lambda}\left(\ln |Q|^{2}\right)_{z \bar{z}}=\frac{2}{\lambda}\left(\frac{Q_{z} \bar{Q}+Q \bar{Q}_{z}}{|Q|^{2}}\right)_{\bar{z}}=\frac{2}{\lambda}\left(\frac{Q_{z}}{Q}\right)_{\bar{z}}=0
$$

Por outro lado, da fórmula da curvatura Gaussiana em um parâmetro conforme (1.55) conseguimos $K=-\frac{1}{\lambda}(\ln \lambda)_{z \bar{z}}$. Daí $-2 K=\Delta \ln \lambda$, e, portanto, $\Delta \ln q^{A R}=-2(-2 K)=4 K$. 
Observação 4.4.1. Toda superfície regular admite uma estrutura de superfície de Riemann, $e$ em toda superfície de Riemann podemos introduzir uma métrica Riemanniana $C^{\infty}$ coerente com a estrutura conforme.

Definição 4.4.3. Seja $R$ uma estrutura de superfície de Riemann de $S$. Usaremos somente as coordenadas $u$ e $v$ tal que $z=u+i v$ é um parâmetro conforme em $R$. Sejam

$$
\begin{aligned}
& A=E d u^{2}+F d u d v+G d v^{2} \\
& B=e d u^{2}+f d u d v+g d v^{2}
\end{aligned}
$$

formas quadráticas reais. Se $A$ é uma forma quadrática real definida em $\Sigma$, escrevemos $R=$ $R_{A}$ quando $A=2 \lambda|d z|^{2}$ em $R$ para alguma função $\lambda$. Neste caso, $A$ é dita uma métrica $R$-conforme. Em $R$ definimos a forma quadrática

$$
Q\left(B, R_{A}\right)=\frac{1}{4}(e-g-2 i f) d z^{2} .
$$

Se $B$ é positiva definida podemos definir em $R_{B}$ a forma quadrática

$$
Q\left(A, R_{B}\right)=\frac{1}{4}(E-G-2 i F) d z^{2} .
$$

Notemos que $Q\left(A, R_{B}\right) \equiv 0$ se, e somente se, $E=G$ e $F=0$

Com esta notação, seja $A$ definida e $(A, B)$ um par fundamental, definiremos a curvatura $H^{\prime}$ (Ver [25]) por $H^{\prime}=H^{\prime}(A, B)=\left(H^{2}-K\right)^{\frac{1}{2}}$ tal que $2 H=\left|k_{2}-k_{1}\right|$ onde $k_{1}$ e $k_{2}$ são as curvaturas principais.

Do fato de que $Q\left(B, R_{A}\right) \equiv 0$ é holomorfa temos um resultado que será essencial para mostrar nosso último teorema.

Lema 4.4.3 ([25]). Suponha $Q\left(B, R_{A}\right) \equiv 0$ holomorfa, com A positiva definida. Então, exceto em pontos isolados, onde $H^{\prime}=0$, seguem as seguintes afirmações:

(i) $H^{\prime} A, H^{\prime} A^{\prime}$ e $W$ são flat, tal que Wé uma forma de linha de curvatura;

(ii) Existem coordenadas $x, y$ tais que $H^{\prime} A=d x^{2}+d y^{2}$ e $H^{\prime} B=\left(H+H^{\prime}\right) d x^{2}+\left(H-H^{\prime}\right) d y^{2}$;

(iii) A função $\log H^{\prime}$ é sub-harmônica em $R_{A}$, se $K(A) \geq 0$, e super-harmônica em $R_{A}$, se $K(A) \leq 0$

(iv) Seja $B$ definida positiva, $\cosh ^{-1}\left(H / H^{\prime}\right)>0$ é super-harmônico em $R_{A}$ se $K(B) \geq 0$, subharmônico em $R_{A}$ se $K(B) \leq 0$, e apenas constante se $K(B) \equiv K(H A) \equiv 0$, enquanto $H / H^{\prime}$ é a própria constante sub-harmônica em $R_{A}$ se $K(B) \leq 0$;

(v) Seja B indefinida, $H / H^{\prime}$ é sub-harmônica em $R_{A}$, se $K(B)>0$ e $H \leq 0$. Super-harmônica em $R_{A}$, se $K(B) \leq 0$ e $H \geq 0$. Apenas constante se $K(B) \equiv K(H A) \equiv 0$. 
Além disso, em todos pontos de $\Sigma$, as seguintes afirmações são válidas:

(vi) Se $B$ é definida positiva, $K(B) \geq\left(\frac{H^{2}}{K}\right) K(H A)$, de modo que $K(B) \geq 0$, onde $K(H A) \geq$ 0, e $K(H A) \leq 0$ onde $K(B) \leq 0$;

(vii) Se $B$ indefinida, $K(B) \leq 0$, onde $K(H A) \geq 0$ e $H<0$, enquanto $K(B) \geq 0$ onde $K(H A) \leq 0$ e $H>0$.

Agora apresentaremos um resultado sobre a classificação de $H$-superfície, em que consiste na função ângulo ser constante. Mas antes temos a seguinte definição:

Definição 4.4.4. Seja $\mathcal{S}_{\kappa, \tau}$ uma familia de $H$-superfície completa, em que $\mathbb{E}(\kappa, \tau), K<0$, satisfaz, para qualquer $\Sigma \in \mathcal{S}_{\kappa, \tau}$,

- $4 H^{2}+\kappa<0$;

- $q$ identicamente nula em $\Sigma \in \mathcal{S}_{\kappa, \tau}$;

- $0<\nu^{2}<1$ é constante ao longo de $\Sigma$;

- $K_{e}=\tau$ e $K=\left(\kappa-4 \tau^{2}\right) \nu^{2}<0$ são constantes.

Teorema 4.4.1 ([17]). Seja $\Sigma \subset \mathbb{E}(\kappa, \tau)$ uma $H$-superfície completa com função ângulo constante. Então $\Sigma$ é ou um cilindro vertical sobre uma curva completa de curvatura $2 H$ em $\mathbb{E}(\kappa, \tau)$, ou um slice em $\mathbb{H}^{2} \times \mathbb{R}$ ou $\mathbb{S}^{2} \times \mathbb{R}$ ou $\Sigma \in \mathcal{S}_{\kappa, \tau}$.

Teorema 4.4.2. Seja $\Sigma \subset \mathbb{E}(\kappa, \tau)$ uma $H$-superfície completa e suponha $q^{A R}$ uma função constante positiva em $\Sigma$. Então $\Sigma$ é um cilindro Hopf sobre uma curva completa de curvatura $2 H$ em $\mathbb{M}^{2}(\kappa)$.

Demonstração. Podemos assumir sem, perda de generalidade, que $\Sigma$ é simplesmente conexo passando o recobrimento universal. Desde que $q^{A R}$ é uma constante positiva, temos $\Delta \ln q^{A R} \equiv 0$. Mas segue do Lema 4.4.2 que $\Delta \ln q^{A R}=4 K$. Logo $K=0$, isto é, a curvatura Gaussiana é identicamente nula em $\Sigma$. Além disso, desde que $q^{A R}=H^{2}-K_{e}\left(I, I I_{A R}\right)=c^{2}>0$ é constante, obtemos que $K_{e}\left(I, I I_{A R}\right)=H^{2}-c^{2}$ é constante em $\Sigma$.

Por outro lado, desde $\Sigma$ é simplesmente conexo, $q^{A R}=c^{2}>0$, então pelo Lema 4.4.3 existe um parâmetro conforme global $z=x+i y$, tal que

$$
c I=d x^{2}+d y^{2} \quad e \quad c I I_{A R}=(H+c) d x^{2}+(H-c) d y^{2} .
$$

Agora combinando a equação de Gauss (4.10) e a expressão de $K_{e}\left(I, I I_{A R}\right)$ dada pelo item 
3 da Proposição 4.3.1, obtemos

$$
\begin{aligned}
\tau^{2}+\left(\kappa-4 \tau^{2}\right) \nu^{2} & =-K_{e}(I, I I) \\
& =-K_{e}\left(I, I I_{A R}\right)+\alpha\left\langle S T_{\theta}, T_{\theta}\right\rangle+\frac{\alpha^{2}|T|^{4}}{4} \\
& =-H^{2}+H^{2}-K_{e}\left(I, I I_{A R}\right)+\alpha\left\langle S T_{\theta}, T_{\theta}\right\rangle+\frac{\alpha^{2}|T|^{4}}{4} \\
& =-H^{2}+c^{2}+\alpha\left\langle S T_{\theta}, T_{\theta}\right\rangle+\frac{\alpha^{2}|T|^{4}}{4} .
\end{aligned}
$$

Reescrevendo,

$$
\frac{\alpha^{2}|T|^{4}}{4}+\left(\kappa-4 \tau^{2}\right)|T|^{2}+\alpha\left\langle S T_{\theta}, T_{\theta}\right\rangle+c^{2}-H^{2}-\tau^{2}-\left(\kappa-4 \tau^{2}\right)=0
$$

em $\Sigma$. Se $T_{\theta}=\theta_{x} \partial_{x}+\theta_{y} \partial_{y}$, então $u=\left\langle T_{\theta}, \partial_{x}\right\rangle$ e $v=\left\langle T_{\theta}, \partial_{y}\right\rangle$. Assim $u=\frac{\theta_{x}}{c}$ e $v=\frac{\theta_{y}}{c}$. Daí,

$$
\begin{aligned}
T_{\theta} & =c\left(u \partial_{x}+v \partial_{y}\right) \\
\left|T_{\theta}\right|^{2} & =c\left(u^{2}+v^{2}\right) \\
\left|T_{\theta}\right|^{4} & =c^{2}\left(u^{2}+v^{2}\right)^{2} .
\end{aligned}
$$

Como $|T|=\left|T_{\theta}\right|$, temos $|T|^{2}=c\left(u^{2}+v^{2}\right) \Rightarrow|T|^{4}=c^{2}\left(u^{2}+v^{2}\right)^{2}$.

Sabemos que $\alpha=\frac{\kappa-4 \tau^{2}}{2 \sqrt{H^{2}+\tau^{2}}}$. Consequentemente $\kappa-4 \tau^{2}=2 \alpha \sqrt{H^{2}+\tau^{2}}$. Então podemos reescrever (4.36) da seguinte forma:

$$
\begin{aligned}
\frac{\alpha^{2}}{4} c^{2}\left(u^{2}+v^{2}\right)^{2}+ & 2 c \alpha \sqrt{H^{2}+\tau^{2}}\left(u^{2}+v^{2}\right)+\alpha\left\langle S T_{\theta}, T_{\theta}\right\rangle \\
+ & c^{2}-\left(H^{2}+\tau^{2}\right)-2 \alpha \sqrt{H^{2}+\tau^{2}}=0 .
\end{aligned}
$$

Multiplicando a igualdade acima por $\frac{2}{\alpha c^{2}}$, obtemos

$$
\begin{array}{r}
\frac{\alpha}{2}\left(u^{2}+v^{2}\right)^{2}+\frac{4 \sqrt{H^{2}+\tau^{2}}}{c}\left(u^{2}+v^{2}\right)+\frac{2}{c^{2}} \alpha\left\langle S T_{\theta}, T_{\theta}\right\rangle \\
\frac{2}{\alpha}-\frac{2}{\alpha c^{2}}\left(H^{2}+\tau^{2}\right)-\frac{4}{c^{2}} \sqrt{H^{2}+\tau^{2}}=0 .
\end{array}
$$

Logo, existe um polinômio nas variáveis $u$ e $v, P(u, v)$, cujo termo principal é $\frac{\alpha}{2}\left(u^{2}+v^{2}\right)^{2}$.

Desde que $\alpha \neq 0$, obtemos $|T|$ é constante em $\Sigma$ e assim, $\nu$ é constante ao longo de $\Sigma$, no qual implica que $\Sigma$ é um cilindro vertical pelo Teorema 4.4.1. 


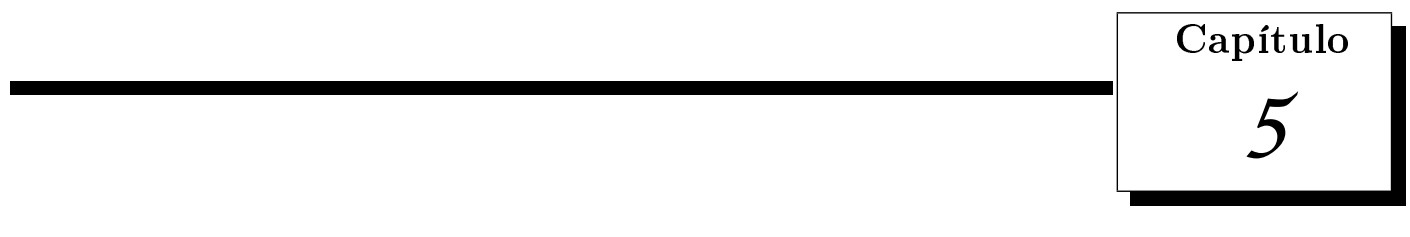

\section{Conclusão}

Neste trabalho, apresentamos aplicações da teoria de pares de Codazzi em superfícies de espaços homogêneos. Mostramos que a técnica é eficiente, tanto nas formas espaciais, onde a primeira e segunda formas fundamentais são pares de Codazzi, quanto nas demais variedades homogêneas tridimensionais de curvatura não-constante, onde os pares de Codazzi são construídos a partir de elementos geométricos da superfície, é aplicado para obter resultados gerais. Nesta conclusão apresentamos um breve resumo dos resultados obtidos.

No capítulo 2, estudamos o comportamento geométrico global das superfícies de curvatura não-positiva. Baseado em imersões de superficies em $\mathbb{R}^{3}$, foi possível estender esse estudo para outros espaços, usando a teoria de pares de Codazzi.

Em $\mathbb{R}^{3}$, temos o seguinte resultado, devido a Efimov, ver [25]:

Teorema: Nenhuma Superfície $\Sigma$ pode ser imersa no espaço Euclidiano $R^{3}$, tal que na métrica induzida $\Sigma$ seja completa e tenha curvatura Gaussiana $K \leq$ constante $<0$.

Destacamos também a conjectura estabelecida por John Milnor em 1966, ver [24]:

Conjectura: Suponha S superfície completa, sem pontos umbilicos, $C^{2}$-imersa em $\mathbb{R}^{3}$ tal que

$$
k_{1}^{2}+k_{2}^{2} \geq c>0
$$

onde $k_{i}, i=1,2$ são as curvaturas principais da imersão e c é uma constante qualquer.

Então, ou a curvatura de Gauss $K$ muda de sinal ou $K \equiv 0$.

Note que no caso de superfície completa tal que a curvatura Gaussiana satifaz $K<0$, a conjectura de Milnor implicaria na existência de pontos onde as duas curvaturas principais tendem simultaneamente a zero. Logo, se certo a conjectura, teremos a generalização do Teorema de Efimov, o qual apenas assegura a existência de pontos onde o produto das curvaturas principais se aproxima de zero. 
O seguinte teorema é uma solução parcial da conjectura de Milnor em $\mathbb{R}^{3}$ no caso de $K \leq 0$ obtida por Smyth-Xavier em 1987, ver [29]:

Teorema: Seja $\psi: S \longrightarrow \mathbb{R}^{3}$ uma superfície completa imersa isometricamente de curvatura não-positiva. Se uma das suas curvaturas principais $k_{i}$ satisfaz $k_{i}^{2} \geq$ const $>$ 0 , então $\psi(S)$ é um cilindro generalizado em $\mathbb{R}^{3}$.

Em particular, os cilindros generalizados são as únicas superfícies completas com curvatura de Gauss não-positivo, em $\mathbb{R}^{3}$ com curvatura média limitada a partir de zero.

Para formas espaciais não Euclidianas, utilizamos a técnica de pares de Codazzi para estudar superfícies completas com curvatura extrínsecas não-positivas. Como consequência da equação de Codazzi, vimos que resultados da teoria de superfícies em $\mathbb{R}^{3}$ continuam valendo nas formas espaciais $\mathbb{H}^{3}$ e $\mathbb{S}^{3}$. Nesse sentido, vimos resultados tipo Milnor e Efimov sobre superfícies completas, imersas em formas espaciais não Euclidianas na qual depende apenas da equação de Codazzi. Assim, apresentamos um resultado geral sobre pares de Codazzi e estendemos este resultado a superfícies em $\mathbb{H}^{3}$ e $\mathbb{S}^{3}$.

Dizemos que as curvaturas principais $k_{1}$ e $k_{2}$ de um par fundamental de $\Sigma$ são estritamente separadas se existe números reais $c_{1}$ e $c_{2}$ tal que

$$
k_{1} \leqslant c_{1}<c_{2} \leqslant k_{2}
$$

Teorema 2.4.1: Seja $(I, I I)$ um par de Codazzi em $\Sigma$ com curvaturas principais estritamente separadas. Se $(\Sigma, I)$ é uma superfície completa com curvatura de Gauss $K(I) \leqslant 0$, então apenas um dos seguintes itens é verificado:

- I é uma métrica plana e $\Sigma$ é homeomorfa ou a um plano, ou a um cilindro ou a um toro plano.

- I não é plana, $\Sigma$ é homeomorfo a um plano e

$$
\int_{\Sigma}|K(I)| d A_{I} \leqslant 2 \pi
$$

Observe também, que se a superfície satisfaz as condições do teorema podemos aplicar o Teorema de Hüber 2.2.2 para obter que $(\Sigma, I)$ tem curvatura total finita. Vemos isso no seguinte resultado:

Teorema: Seja $\Sigma$ uma superfície e $C \subset \Sigma$ um subconjunto compacto. Assuma que $(I, I I)$ é um par de Codazzi sobre $\Sigma \backslash C$ cujas curvaturas principais estão estritamente separadas. Se I é uma métrica completa com curvatura de Gauss não-positiva sobre $\Sigma \backslash C$, então $(\Sigma \backslash C, I)$ tem curvatura total finita. Em particular, $\Sigma$ é do tipo parabólico e tem topologia finita. 
Aplicamos os teoremas anteriores para obter resultados tipos Efimov e Milnor no espaço hiperbólico, $\mathbb{H}^{3}$, e na esfera, $\mathbb{S}^{3}$. Entre outras consequências mostramos também a área dos fins de uma superfície imersa em um espaço modelo tridimensional é finita para a métrica induzida.

Corolário: Seja $\psi: \Sigma \longrightarrow \mathbb{H}^{3}$ uma imersão com curvatura de Gauss $K \leq-1$ e umas das curvaturas principais $k_{i}$ satisfaz $k_{i}^{2} \geq \epsilon^{2}>0$, para alguma constante $\epsilon>0$. Então $\psi$ não é uma imersão completa.

Corolário: Não existem superfícies completas imersas em $\mathbb{H}^{3}$ com curvatura de Gauss $K \leq-\epsilon<0$, para alguma constante $\epsilon>0$, e com curvaturas principais estritamente separadas.

Corolário: Seja $\psi: \Sigma \longrightarrow \mathbb{S}^{3}$ uma imersão com curvatura de Gauss $K \leq$ const $<0$ e umas das curvaturas principais $k_{i}$ satisfaz $k_{i}^{2} \geq \epsilon^{2}>0$, para alguma constante $\epsilon>0$. Então $\psi$ não é uma imersão completa.

Corolário: Seja $\psi: \Sigma \longrightarrow \mathbb{H}^{3}$ uma imersão completa e $C \subset \Sigma$ um subconjunto compacto. Suponha que em $\Sigma \backslash C$ a curvatura de $\psi$ é tal que $k \leq-1$ e umas das curvaturas principais satisfaz $k_{i}^{2} \geq \epsilon^{2}>0$, para alguma constante positiva $\epsilon$. Então $\psi$ tem área finita, $\Sigma$ é do tipo parabólico e tem topologia finita.

Corolário: Seja $\psi: \Sigma \longrightarrow \mathbb{S}^{3}$ uma imersão completa $e C \subset \Sigma$ um subconjunto compacto. Suponha que em $\Sigma \backslash C$ a curvatura de Gauss de $\psi$ satisfaz $K \leq$ const $<0 e$ umas das curvaturas principais $k_{i}$ satisfaze $k_{i}^{2} \geq \epsilon^{2}>0$, para alguma constante $\epsilon>0$. Então $\psi$ tem área finita, $\Sigma$ é do tipo parabólico e tem topologia finita.

Versões melhores desses dois últimos corolários, podem ser obtidos para superfícies em $\mathbb{R}^{3}$. Em [20], foi provado que uma superfície completa em $\mathbb{R}^{3}$ com curvatura de Gauss negativo uniformemente separadas de zero em uma vizinhança do infinito, é topologicamente uma superfície compacta com finito fins, cuja área é finita e cada fins parece cúspides que estende ao infinito, assintótica aos raios (ver também [26]).

Note que a teoria de pares de Codazzi aparece também no estudo de superfícies imersas em espaços ambientes distintos da formas espaciais. Logo, resultados deste capítulo podem ser dados para superfícies espaciais lorentziana tridimensional $\mathbb{L}^{3}$, também imersas em um espaço de dimensão $n$ (semi-Riemanniano) com campo de vetores normal $\xi$ unitário e paralelo, pois a primeira forma fundamental e a segunda forma fundamental associada com $\xi$ constituem um par de Codazzi.

No capítulo 3, aplicamos a teoria de pares de Codazzi em superfícies de $\mathbb{S}^{2} \times \mathbb{R}$ e $\mathbb{H}^{2} \times \mathbb{R}$. O estudo de superfícies, no espaço Euclidiano $\mathbb{R}^{3}$, consiste em sua grande parte de resultados locais, acerca de propriedades da superfície. No entanto, o uso de resultados topológicos, dessas superfícies, permite a obtenção de resultados globais para as mesmas. Entre os vários resultados globais clássicos, destaca-se o Teorema de Liebmann:

Teorema de Liebmann: Seja $\Sigma$ uma superfície compacta de $\mathbb{R}^{3}$, com curvatura constante. Então $\Sigma$ é uma esfera 
Utilizando um corolário do teorema de Bonnet-Myers*, que afirma que uma superfície completa com curvatura Gaussiana constante positiva é compacta, obtemos, pelo Teorema de Liebmann, que a esfera é a única superfície completa com curvatura Gaussiana constante positiva em $\mathbb{R}^{3}$.

O teorema acima nos proporciona o entendimento de como uma superfície Riemanniana completa com curvatura Gaussiana constante positiva pode ser imerso em $\mathbb{R}^{3}, \mathbb{H}^{3}$ ou $\mathbb{S}^{3}$.

Observe que, a partir da equação de Gauss, uma superfície com curvatura de Gauss constante também deve ter curvatura extrínseca constante. Em $\mathbb{R}^{3}$ ambas as curvaturas são iguais, e eles diferem por uma constante em $\mathbb{H}^{3}$ e $\mathbb{S}^{3}$. Além disso, se a curvatura de Gauss é positiva, a curvatura extrínseca do superfície é também positiva em $\mathbb{H}^{3}$ e $\mathbb{R}^{3}$. No entanto, se a curvatura de Gauss $K(I)$ é positivo em $\mathbb{S}^{3}$, então a curvatura extrínseca é somente positiva se $K(I)>1$.

Tendo em conta estas observações, o teorema Liebmann para $\mathbb{M}^{3}(\varepsilon)\left(\operatorname{com} K(I)>1\right.$ em $\left.\mathbb{S}^{3}\right)$ será uma consequência do resultado seguinte [23].

Teorema (Versão Abstrata de Liebmann): Dado $(I, I I) \in \mathcal{P}(\Sigma)$ tal que $I I \in$ $\mathcal{R}(\Sigma)$, entẫo qualquer duas das seguintes condições implica na terceira

(i) $(I, I I)$ é Codazzi

(ii) $K=K(I, I I)>0$ é constante

(iii) A (2,0)-parte de I é holomorfa para uma estrutura conforme dada por II, isto é, $p=I\left(\partial_{z}, \partial_{z}\right)$ é uma função holomorfa para a estrutura conforme induzida por II

Baseado nas referências [5], [6] e [14], apresentamos outra versão do Teorema e Liebmann, onde o espaço ambiente é $\mathbb{M}^{2}(\varepsilon) \times \mathbb{R}$, com $\mathbb{M}^{2}(\varepsilon)=\mathbb{S}^{2}$ quando $\varepsilon=1$, e $\mathbb{M}^{2}(\varepsilon)=\mathbb{H}^{2}$ quando $\varepsilon=-1$. Segue o enunciado do teorema:

Teorema (do tipo Liebmann): Dada uma constante real $K(I)$, existe, a menos de isometria, uma única superfície completa de curvatura Gaussiana constante $K(I)>1$ em $\mathbb{S}^{2} \times \mathbb{R}$ e uma única de curvatura Gaussiana constante $K(I)>0$ em $\mathbb{H}^{2} \times \mathbb{R}$. Além disso, estas superfícies são rotacionalmente simétricas.

Logo, semelhante ao que ocorre em espaços tridimensionais, no ambiente $\mathbb{M}^{2}(\varepsilon) \times \mathbb{R}$, o Teorema tipo Liebmann, classifica as superfícies completas de curvatura constante positiva $K$, a menos de isometria, nesses espaços. Em particular, no ambiente $\mathbb{S}^{2} \times \mathbb{R}$, é mostrado em [6], que não existem superfícies completas com $K \in(0,1)$, como ocorre em $\mathbb{S}^{3}$, ver [13], através do seguinte resultado:

Teorema: Nẫo existem superfícies completas com curvatura Gaussiana constante $K \in$ $(0,1) e m \mathbb{S}^{2}(\varepsilon) \times \mathbb{R}$

A demonstração da existência, no Teorema tipo Liebmann, se faz por meio do estudo das superfícies de revolução completas com curvatura Gaussiana constante em $\mathbb{M}^{2}(\varepsilon) \times \mathbb{R}$. Em

\footnotetext{
${ }^{*}$ Ver [9], cap. IX, seção 3
} 
ambos os casos, tanto em $\mathbb{H}^{2} \times \mathbb{R}$ como em $\mathbb{S}^{2} \times \mathbb{R}$ é possível mostrar que, existe uma única dessas superfícies com curvatura constante positiva, a menos de isometrias do ambiente.

A demonstração da unicidade, é baseada no estudo da forma quadrática $A=I+\frac{1}{\varepsilon K-1} d h^{2}$, definida sobre a superfície, que consiste na composição da métrica da superfície, herdada do ambiente, onde $I$ é a primeira forma fundamental e $h$ é a função altura. Após concluirmos que $A$ é uma métrica Riemanniana, mostramos que:

Teorema: Seja $\Sigma$ uma superfície orientável e $\psi: \Sigma \longrightarrow \mathbb{M}^{2}(\varepsilon) \times \mathbb{R}$ uma imersão de curvatura Gaussiana constante. Se a forma quadrática A é uma métrica Riemanniana então $(A, I I)$ é um par de Codazzi de curvatura extrínseca constante.

Aqui observamos que a existência do par em $\mathbb{M}^{2}(\varepsilon) \times \mathbb{R}$, para imersões de curvatura Gaussiana constante, não depende apenas das equações de Codazzi como acontece em formas espaciais 3dimensionais, mas também depende fortemente da equação de Gauss.

Utilizamos a Versão abstrata do Teorema de Liebmann e o teorema abaixo, ver [6], para a demonstração do Teorema.

Mas antes, através do Teorema de Gauss-Bonnet, temos que $\Sigma$ é uma esfera topológica. A versão abstrata de Liebmann nos leva a conclusão de que a $(2,0)$-parte de $A$ é holomorfa sobre uma esfera topológica e logo é identicamente nula para a estrutura conforme induzida por $I I$.

Teorema: Seja $\Sigma \subset \mathbb{M}^{2} \times \mathbb{R}$ uma imersão de curvatura constante $K>0$ se $\varepsilon=-1$ (resp. $K>1$ se $\varepsilon=1$ ). suponha que $Q d z^{2}$ é identicamente nula em $\Sigma$. Então $\Sigma$ é um pedaço de uma esfera rotacional invariante com curvatura Gaussiana Constante K.

Utilizando o teorema acima, optamos por uma demostração diferente do nosso artigo de referência, [5], no qual podemos utilizar artifícios geométricos enfatizando a importância da teoria de superfície de revolução de curvatura positiva.

Um outro resultado global clássico, é o teorema de Hilbert, no qual fizemos um breve comentário no capitulo passado. Vimos que existem questões em aberto relacionadas como esse teorema, como por exemplo a Conjectura de Milnor. Além disso, obtivemos resultados tipos Efimov no espaço hiperbólico, $\mathbb{H}^{3}$ e na esfera, $\mathbb{S}^{3}$. Novamente, em contraste do que ocorre nos espaços 3dimensionais, o Teorema do tipo Hilbert, ver [5], classifica superfícies completas com curvaturas constante não nula no ambiente $\mathbb{M}^{2}(\varepsilon) \times \mathbb{R}$ e também como nos espaços 3-dimensionais, a ferramenta chave para a demonstração desse Teorema é a teoria de pares de Codazzi. Assim, como no teorema clássico de Hilbert, veremos a inexistência de imersões completas com curvatura constante negativa no espaço produto através do seguinte resultado:

Teorema (do tipo Hilbert): Não existe imersão completa de Curvatura Gaussiana constante $K(I)<-1$ em $\mathbb{H}^{2} \times \mathbb{R} e \mathbb{S}^{2} \times \mathbb{R}$.

Podemos fazer um breve discussão da demonstração, a partir de alguns lemas serão apresentados. Nestes lemas já será possível fazer um paralelo com o caso de superfícies em $\mathbb{R}^{3}$ (teorema de 
Hilbert), ao substituir a condição de curvatura Gaussiana negativa, pela condição de curvatura Gaussiana menos que -1 , para superfícies em $\mathbb{M}^{2}(\varepsilon) \times \mathbb{R}$.

Lema 1 : [3] Seja $\Sigma$ uma superfície orientável e $(A, B)$ um par Codazzi, com curvatura extrínseca $K(A, B)$ negativa e constante tal que A é completa. Então inf $|K(A)| \equiv 0$.

Lema 2: [5] Seja $\Sigma$ uma superfície compacta, com curvatura $K(I)<-1$ em $\mathbb{M}^{2}(\varepsilon) \times \mathbb{R}$. Então A é uma métrica Riemanniana completa.

Lema 3: [5] Seja $\Sigma$ uma superfície orientável e completa tal que $K(I)<-1$ constante em $\mathbb{M}^{2}(\varepsilon) \times \mathbb{R}$. Então inf $|K(A)|>0$.

Esboço da demonstração do Teorema tipo Hilbert. Suponha por contradição que exista imersão $\psi: \Sigma \longrightarrow \mathbb{M}^{2}(\varepsilon) \times \mathbb{R}$ completa de curvatura constante negativa $K(I)<-1$.

Como $K(I)<-1$ então pelo Lema $2 A$ é uma métrica Riemanniana completa e portanto, pelo Teorema 3.4.1 temos $(A, I I)$ é um par Codazzi de curvatura extrínseca $K(A, I I)$ constante. Segue do Lema 3.4.1 que $K(A, I I)=K(I)-\varepsilon<-1-\varepsilon \leq 0$. Logo, pelo Lema 1 , tem-se que inf $|K(A)| \equiv 0$. Mas pelo Lema 3 temos que inf $|K(A)|>0$, o que é contradição. Portanto não existe uma imersão $\psi$ completa com curvatura Gaussiana constante $K(I)<-1$

Alguns estudos mostram a existência ou a não de superfícies completas de curvatura constantes $K(I)$ em $\mathbb{H}^{2} \times \mathbb{R}$ para qualquer número real $K(I)$. No entanto, os casos $-1 \leq K(I)<0$ e $0<K(I)<1$ permanecem abertos em $\mathbb{S}^{2} \times \mathbb{R}$.

No capítulo 4, estudamos pares de Codazzi nos espaços homogêneos $\mathbb{E}(\kappa, \tau)$, baseados no artigo [15]. O objetivo deste capítulo foi encontrar par de Codazzi definido sobre qualquer superfície de curvatura média constante, $H$-superfície em $\mathbb{E}(\kappa, \tau)$, quando $\tau \neq 0$, cuja $(2,0)$-parte é a diferencial Abresch-Rosenberg.

U. Abresch e H. Rosenberg [6] mostraram a existência de uma diferencial quadrática holomorfa em $H$-superfície de $\mathbb{E}(\kappa, \tau)$, isto é, estenderam o conhecido Teorema de Hopf a esses espaços. Classificaram as esferas topológicas com curvatura média constante como uma esfera rotacional simétrica. Apesar da existência de uma diferencial quadrática holomorfa, não existia par de Codazzi em tais $H$-superfícies.

Definição: Dado um parâmetro local conforme z, a diferencial de Abresch-Rosenberg é definida por

$$
\mathcal{Q}^{A R}=Q^{A R} d z^{2}=\left(2(H+i \tau) Q-\left(\kappa-4 \tau^{2}\right) t^{2}\right) d z^{2} .
$$

Além disso, associada a diferencial de Abresch-Rosenberg definimos a função AbreschRosenberg $q^{A R}: \Sigma \longrightarrow[0,+\infty]$ por

$$
q^{A R}=\frac{\left|Q^{A R}\right|}{\lambda^{2}} .
$$


Teorema: Seja $\Sigma$ uma $H$-superfície em $\mathbb{E}(\kappa, \tau)$, então a diferencial AbreschRosenberg $\mathcal{Q}^{A R}$ é holomorfa para uma estrutura induzida pela primeira forma fundamental $I$.

O Teorema acima é a peça fundamental para encontrarmos nosso par de Codazzi. Segue o resultado que garante essa existência.

Teorema (Versão Abstrata de Hopf): Seja (I, II) par fundamental. Então qualquer duas das seguintes condiçôes implica na terceira:

(i) $(I, I I)$ é um par de Codazzi

(ii) $H$ é constante

(iii) A diferencial de Hopf é holomorfa

Em particular, se verificam duas das condiçôes anteriores e $\Sigma$ é uma esfera topológica, o par tem que ser totalmente umbilico.

Podemos redefinir a diferencial diferencial de Abresch-Rosenberg como

$$
Q^{A R} d z^{2}=\left(\left\langle A \partial_{z}, \partial_{z}\right\rangle-\alpha\left\langle T_{\theta}, \partial_{z}\right\rangle^{2}\right) d z^{2}
$$

onde $\alpha=\frac{\kappa-4 \tau^{2}}{2 \sqrt{H^{2}+\tau^{2}}} \in \mathbb{R}$ e $A$ é o operador forma associado a $N$, tal que, a segunda forma fundamental é dada por $I I(X, Y)=\langle A X, Y\rangle, X, Y \in \mathfrak{X}(\Sigma)$. Isto nos leva a seguinte definição:

Definição: Dado uma $H$-superfície $\Sigma \subset \mathbb{E}(\kappa, \tau)$ a segunda forma fundamental Abresch-Rosenberg é definida por

$$
I I_{A R}(X, Y)=I I(X, Y)-\alpha\left\langle T_{\theta}, X\right\rangle\left\langle T_{\theta}, Y\right\rangle+\frac{\alpha|T|^{2}}{2}\langle X, Y\rangle
$$

ou equivalentemente, o operador forma Abresch-Rosenberg $S_{A R}$ é definido por

$$
S_{A R} X=A(X)-\alpha\left\langle T_{\theta}, X\right\rangle T_{\theta}+\frac{\alpha|T|^{2}}{2} X
$$

ou, o traço do operador forma Abresch Rosenberg $S$ é definido por

$$
S X=S_{A R} X-H X=A(X)-\alpha\left\langle T_{\theta}, X\right\rangle T_{\theta}+\frac{\alpha|T|^{2}}{2} X-H X
$$

onde $X, Y \in \mathfrak{X}(\Sigma)$.

Assim, podemos enunciar nosso resultado chave para $H$-superfícies imersas em $\mathbb{E}(\kappa, \tau)$ com $\tau \neq 0$. 
Teorema: Dado uma $H$-superfície $\Sigma \subset \mathbb{E}(\kappa, \tau), H^{2}+\tau^{2} \neq 0$, considere o $(2,0)$-tensor simétrico dado por

$$
I I_{A R}(X, Y)=I I(X, Y)-\alpha\left\langle T_{\theta}, X\right\rangle\left\langle T_{\theta}, Y\right\rangle+\frac{\alpha|T|^{2}}{2}\langle X, Y\rangle
$$

onde

$$
\alpha=\frac{\kappa-4 \tau^{2}}{2 \sqrt{H^{2}+\tau^{2}}} \in \mathbb{R}, \quad e^{2 \theta i}=\frac{H-i \tau}{\sqrt{h^{2}+\tau^{2}}} \quad e \quad T_{\theta}=\cos \theta T+\sin \theta J T
$$

Então, $\left(I, I I_{A R}\right)$ é um par Codazzi com curvatura média constante H. Além disso, a $(2,0)$ - parte de $I I_{A R}$ com respeito a estrutura conforme dada por I assemelha (a menos de uma constante) com a diferencial Abresch-Rosenberg.

Finalizamos nosso trabalho apresentando um resultado simples para $H$-superfícies em $\mathbb{E}(\kappa, \tau)$ dependendo apenas da curvatura assimétrica. Este resultado consiste na classificação de $H$-superfícies com $q^{A R}$ constante.

Teorema: Seja $\Sigma \subset \mathbb{E}(\kappa, \tau)$ uma $H$-superfície completa e suponha $q^{A R}$ uma função constante positiva em $\Sigma$, então $\Sigma$ é um cilindro Hopf sobre uma curva completa de curvatura $2 H$ em $\mathbb{M}^{2}(\kappa)$.

Ressaltamos que $\left(I, I I_{A R}\right)$ ser Codazzi permite calcular a fórmula tipo Simons para $H$-superfícies em $\mathbb{E}(\kappa, \tau)$. Se aplica tal forma, primeiro para estudar o comportamento de $H$-superfícies completa $\Sigma$ de curvatura total Abresch-Rosenberg finita imersa em $\mathbb{E}(\kappa, \tau)$. No caso $H=0$, teorema de Osserman da uma impressionante descrição de $\Sigma$. Se a superfície tem curvatura média diferente de zero e curvatura total finita então deve ser compacta. Em [15], o valor de $H$ é maior do que uma constante positiva dependendo apenas de $\kappa$ e $\tau$. Além disso, juntamente com o princípio máximo de Omori-Yau, é possível classificar $H$-superfície completa, não necessáriamente de curvatura total Abresch-Rosenberg finita, em $\mathbb{E}(\kappa, \tau), \tau \neq 0$.

Aqui apresentaremos a formula tipo Simons para o operador forma $S$ de Abresch-Rosenberg com traço nulo definido em uma $H$-superfície $\Sigma \subset \mathbb{E}(\kappa, \tau), \tau \neq 0$. A fórmula tipo Simons segue do fato de que o operador forma Abresch-Rosenberg de traço nulo é Codazzi e do trabalho de Cheng-Yau [11].

Teorema: Seja $\Sigma$ uma $H$-superficie em $\mathbb{E}(\kappa, \tau)$. Então o operador forma AbreschRosenberg satisfaz

$$
\frac{1}{2} \Delta|S|^{2}=|\nabla S|^{2}+2 K|S|^{2},
$$

ou equivalentemente, $|S|$ diferente de zero, $|S| \Delta|S|-2 K|S|^{2}=|\nabla| S||^{2}$. 


\section{Referências Bibliográficas}

[1] U. Abresch and H. Rosenberg, A Hopf differential for constant mean curvature surfaces in $\mathbb{H}^{2} \times \mathbb{R} e \mathbb{S}^{2} \times \mathbb{R}$, Acta Math. 193 (2004), 141-174.

[2] U. Abresch, H. Rosenberg, Generalized Hopf differential, Mat. Contemp., 28 (2005), 1-28.

[3] A. L. Albujer, J. M. Espinar and L. J. Alías, A Hilbert-type theorem for spacelike surfaces with constant Gaussian curvature in $\mathbb{H}^{2} \times \mathbb{R}_{1}$., Bulletin of the Brazilian Mathematical society, New Series, 40(4):465-478, 2009.

[4] J. A. Aledo, J.M. Espinar, J. A. Gálvez, The Codazzi equation on surfaces., Adv. in Math., 224 (2010), 2511-2530.

[5] J. A. Aledo, J. M. Espinar, J. A. Gálvez, Complete surfaces of constant curvature in $\mathbb{H}^{2} \times \mathbb{R}$ $e \mathbb{S}^{2} \times \mathbb{R}$., Calc. Var. Partial Diferential Equations, 29 (2005), 347-363.

[6] A. Aledo, J. M. Espinar, J. A. Gálvez, Surfaces with constant curvature in $\mathbb{S}^{2} \times \mathbb{R}$ end $\mathbb{H}^{2} \times \mathbb{R}$. Height estimates and representation Bulletin to the Brazilian Mathematical Society, New Series 38(4), (2007), 533 - 554.

[7] H. Alencar, M. do Carmo and R.Tribuzy, A theorem of H. Hopf and athe Cauchy-Riemann inequality, preprint. (Available at http: www.pos.mat.ufal.br/AlencarCarmoTrib.pdf)

[8] M. do Carmo, Geometria Diferencial de Curvas e Superfícies, SBM, Rio de Janeiro (2012).

[9] M. do Carmo, Geometria Riemanniana, IMPA, Rio de Janeiro (2011).

[10] M. do Carmo, Superfícies Minimas, IMPA, Brasil (2003).

[11] S. Y. Cheng, S. T. Yau, Manifolds with constant scalar curvature.Math. Ann., 225 (1977), 195-204.

[12] B. Daniel, Isometric immersions into $\mathbb{H}^{n} \times \mathbb{R} e \mathbb{S}^{n} \times \mathbb{R}$ and applications to minimal surfaces., Preprint. 
[13] B. Daniel, Isometric immersions into 3-dimensional homogenouos manifolds, Comment. Math. Helv. 82 (2007), no. 1, 87-131

[14] J. M. Espinar, La ecuación de Codazzi en superficies. Tesis doctoral, Universidad de Granada, Granada, 2008.

[15] J. M. Espinar, H. A. Trejos, The Abresch-Rosenberg Shape Operator and applications.,

[16] J. M. Espinar, H. Rosenberg, Complete Constant Mean Curvature surfaces and Bernstein type Theorems in $\mathbb{M}^{2} \times \mathbb{R}$, J. Diff. Geom., 82 (2009), $611-628$.

[17] J. M. Espinar, H. Rosenberg, Complete Constant Mean Curvature surfaces in homogeneous spaces, Comm. Math. Helv., 86 (2011), 659-674.

[18] J. M. Espinar, I. S. Oliveira, Locally convex surfaces immersed in a Killing Submersion, Bull. Braz. Math. Soc., 47 (2013) no. 1, 1-17.

[19] H. Farkas, I. Kra, Riemann Surfaces, Springer-Verlag (1991).

[20] J. A. Gálvez, A. Martínez, J. L. Teruel, Complete surfaces with ends of non positive curvature., Advances in Mathematics, 281, (2014), 1202-1215.

[21] J. A. Gálvez, A. Martínez, J. L. Teruel, Complete surfaces with non-positive extrinsic curvature in $\mathbb{H}^{3}$ and $\mathbb{S}^{3}$., J. Math. Anal. Appl., 430, (2015), 1058-1064.

[22] A. Hüber, On subharmonics functions and diferential geometry in the large, Comment. Math. Helv., 32 (1957), 13-72.

[23] T. K. Milnor, Efimov's theorem about Complete Immersed Surfaces of Negative Curvature, Advances in Math., 8(1972),474-543

[24] T. K. Milnor, R. Osserman, Complete surfaces in E3 with constant mean curvature, Commentarii Mathematici Helvetici, 41 (1966-67), 313-318.

[25] T. K. Milnor, Abstract Weingarten Surfaces, J. Diff. Geom., 15 (1980), 365-380.

[26] S. Mendonça, Complete negatively curved immersed ends in $\mathbb{R}^{3}$, preprint, 2014.

[27] B. O’Neill, Semi-Reimannian Geometry, Academic Press (1983).

[28] A. S. Porto, Estimativas de altura e representação para superfícies de curvatura Gaussiana constante em $\mathbb{S}^{2} \times \mathbb{R} e \mathbb{H}^{2} \times \mathbb{R}$. Dissertação (Mestrado em Matemática), Universidade de Brasília, Brasília, 2015.

[29] B. Smyth, F. Xavier, Efimov's theorem in dimension greater than two, Invent. Math., 90 (1987), no. 3, 443-450. 
[30] M. Spivak, A comprehensive Introduction to Differential Geometry,vol 4, Publish or Perish, Inc, Texas(1999).

[31] R. Sacksteder, On hypersurfaces with non-negative sectional curvatures, American Journal of Mathematics, 82(1960), pp. 609-630.

[32] T. Weinstein, An introduction to Lorentz Surfaces., Walter de Gruyter, 1996. 